\title{
Renormalization of Feynman amplitudes on manifolds by spectral zeta regularization and blow-ups.
}

\author{
Nguyen Viet Dang* \\ Bin Zhang ${ }^{\dagger}$
}

\begin{abstract}
Our goal in this paper is to present a generalization of the spectral zeta regularization for general Feynman amplitudes on Riemannian manifolds. Our method uses complex powers of elliptic operators but involves several complex parameters in the spirit of the analytic renormalization by Speer, to build mathematical foundations for the renormalization of perturbative interacting quantum field theories. Our main result shows that spectrally regularized Feynman amplitudes admit an analytic continuation as meromorphic germs with linear poles in the sense of the works of Guo-Paycha and the second author. We also give an explicit determination of the affine hyperplanes supporting the poles. Our proof relies on suitable resolution of singularities of products of heat kernels to make them smooth.

As an application of the analytic continuation result, we use a universal projection from meromorphic germs with linear poles on holomorphic germs to construct renormalization maps which subtract singularities of Feynman amplitudes of Euclidean fields. Our renormalization maps are shown to satisfy consistency conditions previously introduced in the work of Nikolov-TodorovStora in the case of flat space-times.
\end{abstract}

\section{Contents}

1 Introduction.

2 Main results. 6

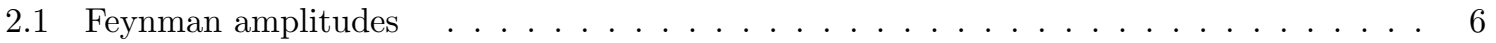

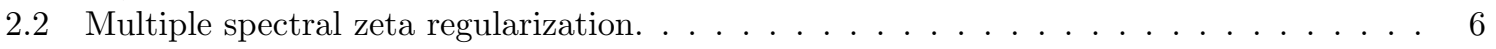

3 Preliminaries. 9

3.1 Meromorphic functions with linear poles. . . . . . . . . . . . . . . 10

3.2 Meromorphic germs of distributions. . . . . . . . . . . . . . . . . 10

3.3 Power expansions of holomorphic germs . . . . . . . . . . . . . . . . . 11

3.4 From Green functions to the heat kernel. . . . . . . . . . . . . . . . . . . 12

3.4 .1 Heat kernels . . . . . . . . . . . . . . . . . . . . . 12

3.5 Local asymptotic expansions of heat kernels $\ldots \ldots \ldots \ldots \ldots \ldots$

4 Reduction of regularized Feynman amplitudes 13

4.1 Holomorphicity of Green's function . . . . . . . . . . . . . . . . . . . 13

4.2 Reduction to local charts and localization near deepest diagonal. . . . . . . . . . . . 14

4.3 Reductions to integrals on cubes. . . . . . . . . . . . . . . . . . . . 15

4.4 Distributional order . . . . . . . . . . . . . . . . . . . . 17

*Institut Camille Jordan (U.M.R. CNRS 5208), Université Claude Bernard Lyon 1, Bâtiment Braconnier, 43, boulevard du 11 novembre 1918, 69622 Villeurbanne Cedex

†School of Mathematics, Yangtze Center of Mathematics, Sichuan University, Chengdu, 610064, P. R. China 
5 Desingularization of parameter space.

5.1 Smoothness problems and the need to resolve singularities. . . . . . . . . . . . 20

5.2 Spanning trees of metric graphs. . . . . . . . . . . . . . . . . 21

5.3 Approximation of the Riemannian distance in normal coordinates. . . . . . . . . . . 222

5.4 Resolving singularities using spanning trees. . . . . . . . . . . . . . . . 23

5.5 Change of variables . . . . . . . . . . . . . . . . . . 25

5.6 Integration by parts, bounding orders and pole decomposition. . . . . . . . . . . . 28

6 Renormalization of Feynman amplitudes 31

6.1 Renormalization maps. . . . . . . . . . . . . . . . . . . . . . 31

6.2 Decompositions of meromorphic germs of distributions $\ldots \ldots \ldots \ldots \ldots \ldots$

6.3 A renormalization map by projections . . . . . . . . . . . . . . 34

7 Appendix: technical details. 35

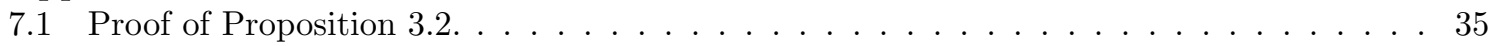

7.2 Products of meromorphic germ of distributions in different variables. . . . . . . . . . 36

7.3 Proof of Lemma $4.1 \ldots \ldots \ldots \ldots \ldots$

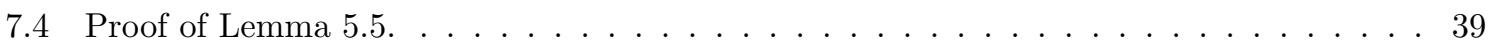

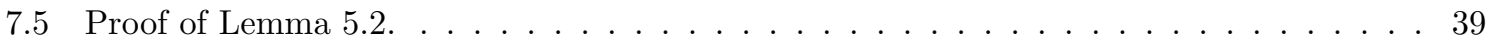

\section{Introduction.}

Zeta regularization. Let $M$ be a smooth, compact, connected manifold without boundary and $P$ be a symmetric, positive, elliptic pseudodifferential operator on $M$. Later on, we will specialize to Schrödinger operators of the form $P=-\Delta_{g}+V$ where $-\Delta_{g}$ is a Laplace operator and $V$ is a smooth nonnegative potential. But the present discussion applies to any symmetric, positive, elliptic pseudodifferential operator $P$. Then $P$ admits a discrete spectral resolution [34, Lemma 1.6.3 p. 51] which means there is an increasing sequence of eigenvalues

$$
\sigma(P)=\left\{0 \leqslant \lambda_{0} \leqslant \lambda_{1} \leqslant \lambda_{2} \leqslant \cdots \leqslant \lambda_{n} \rightarrow+\infty\right\}
$$

and corresponding $L^{2}$-basis of eigenfunctions $\left(e_{\lambda}\right)_{\lambda \in \sigma(P)}$ so that $P e_{\lambda}=\lambda e_{\lambda}$. In his seminal work [69], Seeley constructed the complex powers $\left(P^{-s}\right)_{s \in \mathbb{C}}$ of $P$ as a holomorphic family of linear continuous operators acting on suitable scales of Sobolev spaces on the manifold $M$. In particular for $\operatorname{Re}(s) \geqslant 0$, $P^{-s}$ is bounded in $L^{2}(M)$. Now let us consider the spectral zeta function $\zeta_{P}(s)$ which is defined as the trace $T R\left(P^{-s}\right)$ and coincides with the series :

$$
\zeta_{P}(s)=T R\left(P^{-s}\right)=\sum_{\lambda \in \sigma(P) \backslash\{0\}} \lambda^{-s} .
$$

By Weyl's law on the growth of eigenvalues of $P$ [34, Lemma 1.12 .6 p. 113], the operator $P^{-s}$ is trace class and the series $\zeta_{P}(s)=\sum_{\lambda>0} \lambda^{-s}$ converges as a holomorphic function in $s$ on the halfplane $\operatorname{Re}(s)>\frac{\operatorname{dim}(M)}{\operatorname{deg}(P)}$. Then Seeley showed that $\zeta_{P}(s)$ admits an analytic continuation on the complex plane as a meromorphic function [34, Thm 1.12.2 p. 108] with simple poles. In case $P$ is a differential operator, $\zeta_{P}(s)$ is holomorphic at $s=0$. This result shows one of the first instances of the power of zeta regularization, where we can regularize the divergent series $\sum_{\lambda \in \sigma(P)} 1$ and obtain the value $\zeta_{P}(0)$ of the spectral zeta function $\zeta_{P}$ at $s=0$. More importantly, the residues of $\zeta_{P}(s)$ at its poles can be expressed as multiple of integrals over $M$ of local invariants of the operator $P$ [ 6 , p. 299-303] and are intimately related to the heat invariants of $P$ [34, Thm 1.12 .2 p. 108]. 
From zeta regularization to regularized traces. In the same spirit, zeta regularization techniques were also used in global analysis to construct regularized traces for certain algebras of pseudodifferential operators. The above result of Seeley on the analytic continuation of $T R\left(P^{-s}\right)$ has been generalized to canonical traces on pseudodifferential operators by Kontsevich-Vishik [47, to study anomalies of regularized zeta determinants with related works by Lesch [51] among many authors. Then general types of tracial anomalies were discussed in [53, 17, 58, sometimes in relation with quantum field theory, and finally a general notion of trace for holomorphic families of pseudodifferential operators appears in the work of Paycha-Scott [59]. An important object underlying all these constructions is the notion of noncommutative residue for any pseudodifferential operator $A$. This noncommutative residue can be defined by zeta regularization using complex powers of elliptic operators as follows. Choose any symmetric, positive, elliptic differential operator $P$, then the noncommutative residue of $A$ is defined as the residue at $s=0$ of the meromorphic continuation of the trace $T R\left(A P^{-s}\right)$, and is given by a local formula in the symbol of $A$. In his seminal works, Wodzicki [75, 76] proved that up to constant, this residue is the unique trace on the algebra of pseudodifferential operators. It plays a central role in global analysis and noncommutative geometry. We refer the reader to the monographs [57, 68] for further details on these topics.

Zeta regularization for partition functions. Already in the simple case of spectral zeta functions of the Laplace-Beltrami operator, these regularization methods turn out to be extremely useful to study Euclidean quantum fields on Riemannian manifolds. In the mathematical physics literature, zeta regularization was first applied to quantum field theory on curved spaces by Hawking [41] to give a definition of the partition function of Euclidean QFT. It can also be used to give a mathematical model of the Casimir effect [29]. For topological quantum field theories, following the seminal work of Ray-Singer [63] on analytic torsion, it was soon realized by Schwarz that one can define and calculate the partition function of some abelian BF theories [67] using zeta regularized determinants. Formally, for some flat bundle $(E, \nabla)$ over some smooth compact manifold $M$ of dimension $d$, his formula for the partition function of the BF theory reads

$$
\int_{(A, B) \in \Omega^{k}(M, E) \times \Omega^{n-k-1}(M, E)} \exp \left(-\int_{M} B \wedge d^{\nabla} A\right)=\prod_{k=0}^{d} \operatorname{det}\left(\Delta^{(k)}\right)^{(-1)^{k} k / 2}
$$

where $d^{\nabla}$ is the twisted differential acting on $\Omega^{\bullet}(M, E)$ and the right hand side is the Ray-Singer analytic torsion of the flat bundle $(E, \nabla) \mapsto M$ which is a topological invariant [54, (10) p. 9]. Then Witten generalized the above work of Schwarz by showing that the perturbative partition function of Chern-Simons theory involved the Ray-Singer analytic torsion and also the eta invariant of AtiyahPatodi-Singer. Since the formula looks quite complicated, we refer the reader to [54, (12) p. 9]. But the important point is that the formula involves zeta regularized determinants. The main idea underlying the above results is that partition functions are formally expressed as functional integrals on some space of fields, these partition functions are then identified with regularized determinants of elliptic operators. For instance, in the case of the Dirichlet action functional $S(\varphi)=\frac{1}{2} \int_{M} \varphi\left(-\Delta_{g}\right) \varphi d v(x)$ where $-\Delta_{g}$ is the Laplace-Beltrami operator and $d v$ the Riemannian volume, the partition function $Z$ reads :

$$
Z=\int d \varphi \exp \left(-\frac{1}{2} \int_{M} \varphi\left(-\Delta_{g}\right) \varphi d v\right)=\operatorname{det}\left(-\Delta_{g}\right)^{-\frac{1}{2}}
$$

where $\operatorname{det}\left(-\Delta_{g}\right)$ may be defined as $\exp \left(-\zeta^{\prime}(0)\right)$ where $\zeta$ is the regularized zeta function of the elliptic operator $\left(-\Delta_{g}\right)$ appearing in the definition of the partition function.

For applications in mathematical physics and in the present work, a particular role will be played by complex powers of generalized Laplacians (more generally elliptic, positive, self-adjoint operators of order 2) and their relation with the heat kernel asymptotics. These methods based on the local asymptotic expansion of the heat kernel are crucial in the local index theory [14 and are also used in the works [7, 4, to give a purely spectral definition of the Einstein-Hilbert action functional following [45, 1, 46. 
Another interesting physical property of zeta regularization is its natural covariance which is why it was used in the first place by Hawking. Indeed, for any diffeomorphism $\Phi: M \mapsto M$, the spectrum $\sigma\left(-\Delta_{\Phi^{*} g}\right)$ of $-\Delta_{\Phi^{*} g}$ on $\left(M, \Phi^{*} g\right)$ coincides with the spectrum of $-\Delta_{g}$ on $(M, g)$ and $\sigma\left(-\Delta_{g}\right)$ is thus an invariant of the Riemannian structure $(M, g)$. Therefore zeta regularization is a coordinate independent regularization scheme which depends only on the spectral properties of the Laplacian which in turns is entirely specified by the Riemannian structure $(M, g)$.

Renormalization in quantum field theory. The present paper is written for analysts and does not require any background in physics or quantum field theory. We present our results in a purely mathematical form. However, we felt that for readers with some interest in QFT, it would be preferable to present some physical motivations and the uninterested reader can skip the present paragraph. QFT is a general framework aimed at describing the fundamental forces and particles. In QFT, we are given some graphs called Feynman graphs which pictorially represent complicated interaction process between various particles and we associate to every graph $G$ some number $c_{G}$, called Feynman amplitude, which is often given by some divergent integral as soon as the graph $G$ contains any loops. The issue is that the above zeta regularization methods can only be used to renormalize one loop graphs as discussed in [16, 1.4 p. 10]. For interacting QFT's, it is not enough to regularize only the partition function and one loop graphs, one must renormalize amplitudes whose corresponding graphs contain an arbitrary number of loops. For instance in quantum electrodynamics (QED) which is the QFT describing the interaction of light and matter, the computation of the probability amplitude of some scattering process for two incoming and two outgoing electrons is represented by the following Feynman diagram :

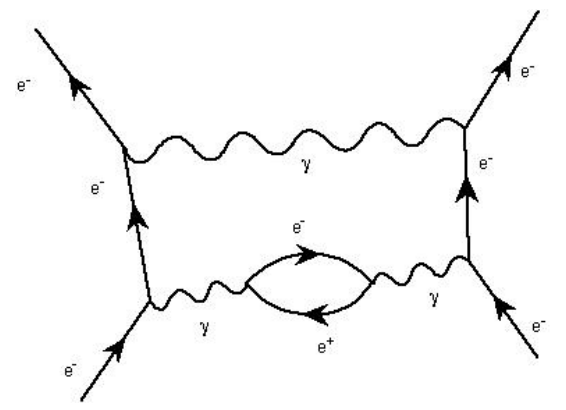

where the electrons are denoted by $e^{-}$, positrons by $e^{+}$and photons by $\gamma$. The corresponding Feynman amplitude is given by some product of electron propagators, represented by the straight lines, and photon propagators represented by the wiggly lines. These propagators are distributions on $\mathbb{R}^{4} \times \mathbb{R}^{4}$ valued in $4 \times 4$ matrices.

For the sake of simplicity, we limit ourselves to scalar theories in the present paper. In these theories, unlike in gauge theories, there is only one scalar valued propagator which is denoted by $\mathfrak{G}$ in the sequel. The topology of the Feynman graphs that we encounter is dictated by the interaction of the theory. For instance in the massless $\phi^{4}$ theory, the only Feynman graphs that we encounter have vertices of degree 4 . Our goal is to use spectral zeta regularization to renormalize multiple loop amplitudes for Euclidean QFT on Riemannian manifolds with the aim to relate them to geometric invariants of Riemannian manifolds which is the subject of future work of the authors. Our starting point is the work of Eugene Speer on analytic renormalization in QFT [70, 71, 72] who found an alternative formulation of the usual BPHZ renormalization algorithm, based on analytic regularization with several complex parameters. The analytic structure of the regularized amplitude in these variables encodes rich algebraic structure so that a renormalized amplitude may be defined by the application of a universal projector, independent of the graph in question, to the regularized amplitude. Indeed, we will show that regularized amplitudes are meromorphic germs with linear poles and in subsection 6.3 .

\footnotetext{
${ }^{1}$ The space of Riemannian structure if the set of pairs $(M, g)$ quotiented by isometries
} 
we will describe a straightforward way of subtracting the divergent part of the regularized amplitudes while keeping only the holomorphic part. Then renormalization will be reformulated in definition 6.4 as the evaluation at some poles of the holomorphic part of the regularized amplitude. This projection is a useful substitute to the BPHZ algorithm and the method pioneered by Connes-Kreimer based on Hopf algebras and Birkhoff factorizations. In our work, a common point with the BPHZ algorithm and Speer's work, is that we rely on Hepp sectors and resolution of singularities arguments.

Let us show how the idea of analytic renormalization works in an example on flat space. On Euclidean space $\mathbb{R}^{4}$, the Green function of the Laplace operator reads $\mathfrak{G}(x, y)=C Q^{-1}(x-y)$ where $C$ is some constant and $Q$ is the quadratic form $Q(v)=\sum_{i=1}^{4} v_{i}^{2}$. On configuration space $\left(\mathbb{R}^{4}\right)^{6}$, the Feynman rules assign to the graph

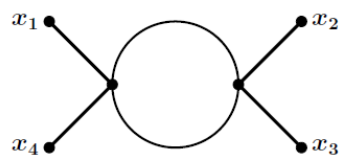

the corresponding amplitude

$$
T\left(x_{1}, x_{2}, x_{3}, x_{4}\right)=\int_{\left(y_{1}, y_{2}\right) \in\left(\mathbb{R}^{4}\right)^{2}} \mathfrak{G}\left(x_{1}, y_{1}\right) \mathfrak{G}\left(x_{2}, y_{1}\right) \mathfrak{G}^{2}\left(y_{1}, y_{2}\right) \mathfrak{G}\left(y_{2}, x_{3}\right) \mathfrak{G}\left(y_{2}, x_{4}\right) d^{4} y_{1} d^{4} y_{2},
$$

which is given by some formal product of Green functions. To get rid of the infrared divergence due to the fact that we integrate in some infinite volume $\left(\mathbb{R}^{4}\right)^{2}$, one may either introduce a sharp cut-off by replacing $\mathbb{R}^{4}$ by a finite box, or we may as well insert some smooth compactly supported cut-off function $g \in C_{c}^{\infty}\left(\mathbb{R}^{4}\right)$ for each variable $\left(y_{i}\right)_{i \in\{1,2\}}$ corresponding to the internal vertices of the Feynman graph as follows :

$$
T\left(x_{1}, x_{2}, x_{3}, x_{4}\right)=\int_{\left(\mathbb{R}^{4}\right)^{2}} \mathfrak{G}\left(x_{1}, y_{1}\right) \mathfrak{G}\left(x_{2}, y_{1}\right) \mathfrak{G}^{2}\left(y_{1}, y_{2}\right) \mathfrak{G}\left(y_{2}, x_{3}\right) \mathfrak{G}\left(y_{2}, x_{4}\right) g\left(y_{1}\right) g\left(y_{2}\right) d^{4} y_{1} d^{4} y_{2} .
$$

In fact, it is natural to view the full amplitude $\mathfrak{G}\left(x_{1}, y_{1}\right) \mathfrak{G}\left(x_{2}, y_{1}\right) \mathfrak{G}^{2}\left(y_{1}, y_{2}\right) \mathfrak{G}\left(y_{2}, x_{3}\right) \mathfrak{G}\left(y_{2}, x_{4}\right)$ as a distribution in $\mathcal{D}^{\prime}\left(\left(\mathbb{R}^{4}\right)^{6}\right)$, so we may think that we insert some smooth compactly supported cut-off function $g\left(y_{1}\right) g\left(y_{2}\right)$ on $\left(\mathbb{R}^{4}\right)^{2}$ so that $T\left(x_{1}, x_{2}, x_{3}, x_{4}\right)$ is well-defined as the pushforward of the product $\mathfrak{G}\left(x_{1}, y_{1}\right) \mathfrak{G}\left(x_{2}, y_{1}\right) \mathfrak{G}^{2}\left(y_{1}, y_{2}\right) \mathfrak{G}\left(y_{2}, x_{3}\right) \mathfrak{G}\left(y_{2}, x_{4}\right) g\left(y_{1}\right) g\left(y_{2}\right) d^{4} y_{1} d^{4} y_{2}$ along the fibers of the projection $\left(\mathbb{R}^{4}\right)^{6} \mapsto\left(\mathbb{R}^{4}\right)^{4}$.

In terms of the quadratic function $Q$, the above amplitude reads :

$$
\begin{aligned}
\int_{\left(y_{1}, y_{2}\right) \in\left(\mathbb{R}^{4}\right)^{2}} & Q^{-1}\left(x_{1}, y_{1}\right) Q^{-1}\left(x_{2}, y_{1}\right) Q^{-1}\left(y_{1}, y_{2}\right) Q^{-1}\left(y_{1}, y_{2}\right) \\
& \times Q^{-1}\left(y_{2}, x_{3}\right) Q^{-1}\left(y_{2}, x_{4}\right) g\left(y_{1}\right) g\left(y_{2}\right) d^{4} y_{1} d^{4} y_{2} .
\end{aligned}
$$

Now for each $Q^{-1}$ in factor in the amplitude, we shall introduce a complex power $s$ as follows :

$$
\begin{array}{r}
T(\mathbf{s})=\int_{\left(y_{1}, y_{2}\right) \in\left(\mathbb{R}^{4}\right)^{2}} Q^{-s_{1}}\left(x_{1}, y_{1}\right) Q^{-s_{2}}\left(x_{2}, y_{1}\right) Q^{-s_{3}}\left(y_{1}, y_{2}\right) Q^{-s_{4}}\left(y_{1}, y_{2}\right) \\
\times Q^{-s_{5}}\left(y_{2}, x_{3}\right) Q^{-s_{6}}\left(y_{2}, x_{4}\right) g\left(y_{1}\right) g\left(y_{2}\right) d^{4} y_{1} d^{4} y_{2}
\end{array}
$$

where the new amplitude depends on $\mathbf{s}=\left(s_{1}, \ldots, s_{6}\right) \in \mathbb{C}^{6}$. For $\operatorname{Re}\left(s_{i}\right)_{1 \leqslant i \leqslant 6}$ large enough, one can easily see that the amplitude defining $T$ is integrable. The main result of Speer is the fact that $T(\mathbf{s})$ admits an analytic continuation in $\mathbf{s} \in \mathbb{C}^{6}$ as a meromorphic function with linear poles. Then he shows that $T(\mathbf{s})$ decomposes as the sum of a singular part and a holomorphic part at $\mathbf{s}=(1, \ldots, 1) \in \mathbb{C}^{6}$ and renormalization consists in subtracting the singular part and evaluating at $(1, \ldots, 1) \in \mathbb{C}^{6}$. 
The main goal of the present paper is to combine the methods from zeta regularization to present a generalization of analytic renormalization to general Riemannian manifolds. Then we will show that the renormalization defined satisfies the consistency axioms of Nikolov-Todorov-Stora in [56] inspired by the seminal works of Epstein-Glaser [28].

\section{Main results.}

In the present section, we introduce the main objects of study and state the main results of our work. We define first Feynman amplitudes, next we explain how to implement a zeta regularization with several complex parameters then we state the first main analytic continuation Theorem and we finally give a simplified version of our second main theorem concerning applications of the analytic continuation result to renormalization in QFT.

\subsection{Feynman amplitudes}

We work on a compact, connected Riemannian manifold $(M, g)$ without boundary, the LaplaceBeltrami operator is denoted by $\Delta_{g}$ and $C_{\geqslant 0}^{\infty}(M)$ denotes smooth, nonnegative functions on $M$. For a potential $V \in C_{\geqslant 0}^{\infty}(M)$, it is well-known that the Schrödinger operator $P=-\Delta_{g}+V$ is a second order, symmetric, positive, elliptic differential operator which defines a unique unbounded, self-adjoint operator acting on $L^{2}(M)$ [33, p. 34-35]. We now generalize the Feynman rules to this case. That is, to every graph we associate a formal product of Green kernels of the operator $P$. Since on a general manifold, there is no Fourier transform, our Feynman rules are just the Riemannian versions of the Euclidean Feynman rules in position space of [18, definition 2.1] (see also [23]).

Definition 2.1 (Feynman rules) Let $\mathfrak{G}(x, y)$ denote the Green kernel of the operator $P$, then for a graph $G$ with the set of vertices $V(G)$ and the set of edges $E(G)$, if for any edge $e \in E(G)$, the vertices incident to $e$ are $i(e)$ and $j(e)$ and $G$ has no self-loops, then the Feynman amplitude associated to $G$ is defined as

$$
t_{G}=\prod_{e \in E(G)} \mathfrak{G}\left(x_{i(e)}, x_{j(e)}\right)
$$

as a $C^{\infty}$ function on $M^{V(G)} \backslash\{$ all diagonals $\}$.

Remark 2.1 Since the Green kernel $\mathfrak{G}$ is symmetric in its variables, $t_{G}$ is well-defined. The graphs are not allowed to have self-loops since the Green function $\mathfrak{G}$ is not well-defined on the diagonal hence cannot be evaluated at coinciding points. The above Feynman rules correspond to a perturbative Euclidean QFT where the Lagrangian is already Wick renormalized which explains why self-loops (also called tadpoles in the physics literature) are excluded.

\subsection{Multiple spectral zeta regularization.}

The operator $P^{-s}$ is defined as a spectral function of the operator $P$ in a very simple way following 34 , equation (1.12.13) p. 107] :

Definition 2.2 (Complex powers) For $\operatorname{Re}(s) \geqslant 0$, for every $u \in L^{2}(M)$, decompose $u$ in the orthonormal basis $\left(e_{\lambda}\right)_{\lambda}$ of $L^{2}(M)$ given by the eigenfunctions of $P$. Then

$$
P^{-s} u=\sum_{\lambda \in \sigma(P) \backslash\{0\}} \lambda^{-s}\left\langle u, e_{\lambda}\right\rangle e_{\lambda}
$$

where the sum on the right hand side converges absolutely in $L^{2}(M)$ since the eigenvalue $\lambda_{i} \underset{i \rightarrow+\infty}{\longrightarrow}+\infty$ hence the sequence $\left(\lambda_{i}^{-s}\right)_{i}$ remains bounded. 
The Schwartz kernel of $P^{-s}$ is then by definition :

$$
\mathfrak{G}^{s}(x, y)=\sum_{\lambda \in \sigma(P) \backslash\{0\}} \lambda^{-s} e_{\lambda}(x) e_{\lambda}(y)
$$

where we abusively denoted by $\mathfrak{G}^{s}(x, y)$ an actual distribution $\mathfrak{G}^{s} \in \mathcal{D}^{\prime}(M \times M)$ and the series on the r.h.s. converges in $\mathcal{D}^{\prime}(M \times M)$. We will later see that $\mathfrak{G}^{s}$ is actually a function on $M \times M$ for $\operatorname{Re}(s)>\frac{d}{2}$ where $d=\operatorname{dim}(M)$. We shall generalize this regularization to the case $M=\mathbb{R}^{d}$ with flat Euclidean metric $g$ and $P=-\Delta_{g}+m^{2}, m \in \mathbb{R}_{\geqslant 0}$. Our definition of $\mathfrak{G}^{s}$ in the flat case is similar to the compact case since we define $\mathfrak{G}^{s}$ with complex powers of the Laplace operator :

Definition 2.3 (Complex powers for flat space.) If $M=\mathbb{R}^{d}, g$ is a constant quadratic form and $m \in \mathbb{R}_{\geqslant 0}$ is a mass, then we set :

$$
\mathfrak{G}^{s}(x, y)=\frac{1}{(2 \pi)^{d}} \int_{\mathbb{R}^{d}} \frac{e^{i\langle(x-y), \xi\rangle}}{\left(g^{\mu \nu} \xi_{\mu} \xi_{\nu}+m^{2}\right)^{s}} d^{d} \xi=\frac{1}{\Gamma(s)} \int_{0}^{+\infty} \frac{1}{(4 \pi t)^{\frac{d}{2}}} e^{-\frac{\langle x-y, x-y\rangle_{g}}{4 t}} e^{-t m^{2}} t^{s-1} d t .
$$

It is immediate from the above formulas that $\mathfrak{G}^{s}$ is the Schwartz kernel of $\left(-\Delta_{g}+m^{2}\right)^{-s}$ and that when $s=1$, we recover the Green function of the operator $\left(-\Delta_{g}+m^{2}\right)$.

Definition 2.4 (Regularized Feynman rules) Under the above assumptions, we denote by $P^{-s}$ the complex powers of $P$ and by $\mathfrak{G}^{s}(x, y) \in \mathcal{D}^{\prime}(M \times M)$ the corresponding Schwartz kernel. Then for a graph $G$ with vertex set $V(G)$ and edge set $E(G)$, the regularized Feynman amplitude reads

$$
t_{G}(s)=\prod_{e \in E(G)} \mathfrak{G}^{s_{e}}\left(x_{i(e)}, x_{j(e)}\right)
$$

which is in $C^{\infty}\left(M^{V(G)} \backslash\{\right.$ all diagonals $\left.\}\right)$.

Remark 2.2 We will see later in Lemma 4.1 that $\mathfrak{G}^{s}$ is actually in $C^{k}(M \times M)$ for Re(s) large enough hence it follows that the above Feynman rules also make sense for graphs $G$ with self-loops when $\operatorname{Re}(s)$ is large enough which was not true for $s=1$ since $\mathfrak{G}$ would be a distribution singular on the diagonal.

Let us state our first main Theorem :

Theorem 2.1 Let $(M, g)$ be a smooth, compact, connected Riemannian manifold without boundary of dimension $d, d v(x)$ the Riemannian volume and $P=-\Delta_{g}+V, V \in C_{\geqslant 0}^{\infty}(M)$ or $M=\mathbb{R}^{d}$ with a constant metric $g$ and $P=-\Delta_{g}+m^{2}, m \in \mathbb{R}_{\geqslant 0}$. Then for every graph $G$, on the configuration space $\left(M^{V(G)}, g_{V(G)}\right)$ endowed with the product metric $g_{V(G)}$, and product volume form $d v_{M^{V(G)}}$, for any test function $\varphi \in C^{\infty}\left(M^{V(G)}\right)$,

$$
s \mapsto \int_{M^{V(G)}} t_{G}(s) \varphi d v_{M^{V(G)}}
$$

can be analytically continued near $\left(s_{e}=1\right)_{e \in E(G)}$ as a meromorphic germ with possible linear poles on the hyperplanes of equation $\sum_{e \in G^{\prime}} s_{e}-\left|E\left(G^{\prime}\right)\right|=0$ where $G^{\prime}$ is a subgraph of $G$ such that $2\left|E\left(G^{\prime}\right)\right|-b_{1}\left(G^{\prime}\right) d \leqslant 0,\left|E\left(G^{\prime}\right)\right|$ is the number of edges in $G^{\prime}$ and $b_{1}\left(G^{\prime}\right)$ the first Betti number of $G^{\prime}$.

To recover renormalized Feynman amplitudes, we follow the strategy of [24, 2.2]. We cannot evaluate $t_{G}(s)$ at $\left(s_{e}=1\right)_{e \in E(G)}$ since it might belong to the polar set of $t_{G}$. However, applying the machinery from [38] allows us to subtract the polar part of $t_{G}(s)$ at $\left(s_{e}=1\right)_{e \in E(G)}$ while keeping a holomorphic part. This is based on an extension of the framework of 38] to distributions valued in 
meromorphic germs with linear poles constructed in paragraph 6.2. Then to recover the renormalized Feynman amplitude, it suffices to evaluate the holomorphic part at $\left(s_{e}=1\right)_{e \in E(G)}$. Following Speer [71, section 3], analytic renormalization will be reformulated in definition 6.4 as the evaluation at some poles of the holomorphic part of the regularized amplitude. This idea was recently abstracted in the works [19, 39] in a purely algebraic way where the composition of a projection on the holomorphic part and the evaluation at $\left(s_{e}=1\right)_{e \in E(G)}$ is called evaluator [39, 1.3 p. 6]. The renormalization $\mathcal{R}\left(t_{G}\right)$ of some amplitude $t_{G}$ is the composition of the operations summarized in the following diagram :

$\left.t_{G} \stackrel{\text { regularization }}{\longrightarrow} t_{G}(s) \stackrel{\text { projection on holomorphic part }}{\longrightarrow} \pi\left(t_{G}(s)\right) \stackrel{\text { evaluation at } s=s_{0}}{\longrightarrow} \mathbf{e v}\right|_{s_{0}}\left(\pi\left(t_{G}(s)\right)\right)=\mathcal{R}\left(t_{G}\right)$,

where $s_{0}=\left(s_{e}=1\right)_{e \in E(G)}$.

In section 6, we apply the above ideas to the renormalization of quantum field theories on Riemannian manifolds and show the existence of a collection of renormalization maps $\left(\mathcal{R}_{M^{I}}\right)_{I \subset \mathbb{N}}$ that roughly assign to each graph $G$ a renormalized amplitude in $\mathcal{D}^{\prime}\left(M^{V(G)}\right)$ such that the renormalization maps satisfy the consistency axioms 6.2 which come from the work of Nikolov-Todorov-Stora [56]. Let us state a simplified version of our second main Theorem 6.2 :

Theorem 2.2 Let $(M, g)$ be a smooth, compact, connected Riemannian manifold without boundary of dimension $d, d v(x)$ the Riemannian volume and $P=-\Delta_{g}+V, V \in C_{\geqslant 0}^{\infty}(M)$ or $M=\mathbb{R}^{d}$ with a constant metric $g$ and $P=-\Delta_{g}+m^{2}, m \in \mathbb{R}_{\geqslant 0}$. Then for every graph $G$, on the configuration space $M^{V(G)}$ endowed with the product volume form $d v_{M^{V(G)}}$ :

- there exists distributions $\pi\left(t_{G}(s)\right),(1-\pi)\left(t_{G}(s)\right)$ such that for any test function $\varphi \in$ $C^{\infty}\left(M^{V(G)}\right)$, we have a unique decomposition

$$
\int_{M^{V(G)}} t_{G}(s) \varphi d v_{M^{V(G)}}=\underbrace{\int_{M^{V(G)}}(1-\pi)\left(t_{G}(s)\right) \varphi d v_{M^{V(G)}}}_{\text {meromorphic germ }}+\int_{M^{V(G)}} \pi\left(t_{G}(s)\right) \varphi d v_{M^{V(G)}}
$$

where $s \mapsto \int_{M^{V(G)}} \pi\left(t_{G}(s)\right) \varphi d v_{M^{V(G)}}$ is a holomorphic germ at $s_{0}=\left(s_{e}=1\right)_{e \in E(G)} \in$ $\mathbb{C}^{|E(G)|}$.

- If $\varphi \in C_{c}^{\infty}\left(M^{V(G)} \backslash\right.$ all diagonals $)$ then

$$
\lim _{s \rightarrow s_{0}} \int_{M^{V(G)}} \pi\left(t_{G}(s)\right) \varphi d v_{M^{V(G)}}=\int_{M^{V(G)}} t_{G} \varphi d v_{M^{V(G)}}
$$

which means $\lim _{s \rightarrow s_{0}} \pi\left(t_{G}(s)\right)$ is a distributional extension of $t_{G} \in C^{\infty}\left(M^{V(G)} \backslash\right.$ all diagonals).

Then the reader is referred to Theorem 6.2 where we prove many important properties enjoyed by the renormalized amplitudes $\lim _{s \rightarrow s_{0}} \pi\left(t_{G}(s)\right)$. The most important being a factorization equation appearing in definition 6.2 which translates in mathematical terms the essential property of locality in Euclidean QFT.

Related works. In recent works of Hairer [40] and Pottel [61, 62, the authors give analytic treatments of the BPHZ algorithm. Like in the present paper, they also start from Feynman amplitudes in position space but Hairer works on $\mathbb{R}^{d}$ with abstract kernels $K$ with specific singularity along diagonals, whereas we work on Riemannian manifolds but we limit our discussion to Green kernels of Laplace type operators. He also uses Hepp sectors to perform some kind of multiscale analysis to analyze the divergences of the Feynman amplitudes. It would be interesting to compare the renormalization maps defined in the present paper with the valuations in Hairer's paper [40] and definition 6.2 with the consistency axioms of [40]. 
Our treatment of renormalization bears a strong inspiration from the seminal work of EpsteinGlaser [28] who were among the first to understand the central role of causality (this is replaced in the current work by locality) in perturbative renormalization. Their work was generalized by Brunetti-Fredenhagen [15] to curved space times while the crucial physical notions of covariance of the renormalization were adressed in the works of Hollands-Wald 43, 44. A recent investigation of the Epstein-Glaser renormalization using resolution of singularities can be found in the thesis of Berghoff 11, 12, clarifying some previous attempts [9, 10. Our results seem to be more general since we work in the manifold case and we resolve singularities by hand instead of using the compactifications of configuration space of Fulton-McPherson and de Concini-Procesi.

There is a famous interpretation of the BPHZ renormalization in terms of Hopf algebras pioneered by Connes-Kreimer [20, 21, 22]. This approach using dimensional regularization works essentially in momentum space and does not generalize in a straightforward way to curved spaces. Motivated by problems from number theory, Marcolli-Ceyhan [18] managed to reformulate the Hopf-algebraic approach on configuration space.

Renormalization in the Riemannian setting was recently discussed in the book by Costello [23], however it seems his proof of subtraction of counterterms contains some gaps that were fixed by Albert who also extended Costello's work to manifolds with boundary [2, 3].

Perspectives. A natural extension of our results would be to prove an analytic continuation result for Feynman amplitudes made from Schwartz kernels of holomorphic families of pseudodifferential operators in the sense of Paycha-Scott [59] generalizing the Schwartz kernels of complex powers of Laplace operators. For the sake of simplicity, we limited ourselves to complex powers of Laplace operators because of their explicit relation with heat kernels and leave it to another work for the investigation of the more general case. Another interesting situation is when the manifold $M$ is noncompact with specific asymptotic structure as in scattering theory. Probably in this case, we would need to use resolvents to define complex powers.

It would also be very interesting to test our proof in the Lorentz case with the Feynman propagator instead of the Green's function of the Laplacian. Then a natural question would be what is the substitute in the Lorentz case for the complex powers of the Laplace operators? The first author defined complex regularization of Feynman propagators in some previous work 24] on analytic Lorentzian space-times under some very restrictive assumptions of geodesic convexity. This was based on the Hadamard parametrix for the Feynman propagator. From our point of view, it would be preferable to define some complex regularization scheme on smooth Lorentzian space-times which are not necessarily geodesically convex. The scheme should be manifestly covariant as spectral regularization on Riemannian manifolds. Probably, this would be based on the recent results of [8, 30, 31, 33, 25, 26, 74, on the analytic structure of Feynman propagators. Another interesting direction is to investigate if it is possible to renormalize the amplitudes in Euclidean theory then perform a geometric Wick rotation as in Gérard-Wrochna 32 to build renormalized amplitudes of the corresponding Lorentzian QFT.

Acknowledgements. We would like to thank M. Wrochna, D. Häfner, Y. Colin de Verdière, E. Herscovich, for many interesting comments they made to one of us (NVD) when we first presented this work in Grenoble. Also big thanks to C. Guillarmou and S. Paycha for answering many of our questions on regularization using pseudodifferential powers. The first author wants to thank Dang Nguyen Bac for many discussions related to blow-ups and algebraic geometry. Finally, NVD acknowledges the ANR-16-CE40-0012-01 grant for financial support.

\section{Preliminaries.}

The goal of the present section is to introduce the language of meromorphic germs with linear poles and give the main definitions since meromorphic germs appear in the formulation of Theorem 2.1 . We also introduce their distributional counterpart which we call meromorphic germs of distributions 
which is the fundamental object needed for the proof of Theorem 2.1. The meromorphic germs of distributions are essentially distributions depending on some parameter $s \in \mathbb{C}^{p}, p \in \mathbb{N}$, such that when they are paired with some test function $\varphi$, they give meromorphic germs in $s$.

\subsection{Meromorphic functions with linear poles.}

In this paper, all meromorphic functions of several variables $s=\left(s_{1}, \ldots, s_{p}\right) \in \mathbb{C}^{p}$ have singularities along unions of affine hyperplanes. In fact, we will work with meromorphic germs with linear poles in the terminology of 38 . We work in the space $\mathbb{R}^{p}$, and with the standard complex structure on $\mathbb{C}^{p}=\mathbb{R}^{p} \otimes \mathbb{C}$, let $\left(\mathbb{R}^{p}\right)^{*}$ be the dual. In the sequel, holomorphic functions on some domain $\Omega \subset \mathbb{C}^{p}$ and holomorphic germs at $s_{0} \in \mathbb{C}^{p}$ are denoted by $\mathcal{O}(\Omega)$ and $\mathcal{O}_{s_{0}}\left(\mathbb{C}^{p}\right)$ respectively.

Definition 3.1 (meromorphic germs) Let $s_{0} \in \mathbb{R}^{p}$, then $f$ is a meromorphic germ with (real) linear poles at $s_{0}$ if there are vectors $\left(L_{i}\right)_{1 \leqslant i \leqslant m}$ in $\left(\mathbb{R}^{p}\right)^{*}$, such that

$$
\left(\prod_{i=1}^{m} L_{i}\left(.-s_{0}\right)\right) f \in \mathcal{O}_{s_{0}}\left(\mathbb{C}^{p}\right) .
$$

Meromorphic germs with linear poles at $s_{0} \in \mathbb{C}^{p}$ are denoted by $\mathcal{M}_{s_{0}}\left(\mathbb{C}^{p}\right)$.

Geometrically such a meromorphic germ $f$ is singular along some arrangement of affine hyperplanes $\left\{s \in \mathbb{C}^{p} \text { s.t. } L_{i}\left(s-s_{0}\right)=0\right\}_{1 \leqslant i \leqslant m}$, intersecting at the point $s_{0}$.

\subsection{Meromorphic germs of distributions.}

In this paper, we deal with families of distributions $t(s)$ on some smooth second countable manifold $X$ without boundary, depending meromorphically on some parameter $s$ and whose poles are linear. We will also call them distributions valued in meromorphic germs with linear poles and will denote the space of such families by $\mathcal{D}^{\prime}(X, \mathcal{M})$. We devote this subsection to their proper definition. Our plan is to give the definition gradually starting from holomorphic objects. For a smooth manifold $X$ with given smooth density $d v$, we will use $\mathcal{D}^{\prime}(X)$ to denote the space of distributions on $X$ and $\mathcal{D}^{\prime}(X)$ is defined in the present paper as the topological dual of $C_{c}^{\infty}(X) \otimes d v$ which is the space of smooth, compactly supported densities. But in many situations where the density is explicitely given by the geometric problem, we may equivalently think of distributions as the dual of $C_{c}^{\infty}(X)$.

Holomorphic families of distributions. Before we discuss meromorphic germs of distributions, let us start smoothly by defining distributions depending holomorphically on some extra parameter.

Definition 3.2 (holomorphic families) Let $\Omega \subset \mathbb{C}^{p}$ be a complex domain, and $X$ be a smooth manifold, a holomorphic family of distributions on $X$ parametrized by $\Omega$ is a family $(t(s))_{s \in \Omega}$ of distributions on $X$, such that for every test function $\varphi \in C_{c}^{\infty}(X), s \mapsto\langle t(s), \varphi\rangle$ defines a holomorphic function on $\Omega$. Such set of holomorphic families of distributions will be denoted by $\mathcal{D}^{\prime}(X, \mathcal{O}(\Omega))$.

Then we next introduce a variant of the above definition involving distributions whose distributional order is bounded by some integer $m$.

Definition 3.3 (holomorphic families with bounded order) Let $m$ be an integer and $X$ a smooth manifold, then a distribution $t$ is of order bounded above by $m$ on $X$ if $t$ defines a continuous linear function on $C_{c}^{m}(X)$. For a complex domain $\Omega$, a holomorphic family of distributions $(t(s))_{s \in \Omega}$ of order bounded above by $m$ on $X$, is a family $(t(s))_{s \in \Omega}$ of distributions of order bounded above by $m$ such that for every test function $\varphi \in C_{c}^{m}(X), s \mapsto\langle t(s), \varphi\rangle$ defines a holomorphic function on $\Omega$. This set is denoted by $\mathcal{D}^{\prime, m}(X, \mathcal{O}(\Omega))$. 
Once we defined holomorphic families of distributions where the complex parameter lives on some domain $\Omega$ containing some element $s_{0}$, it is natural to give a definition where we want to forget the information about $\Omega$ and localize around $s_{0}$. We thus work at the level of holomorphic germs near $s_{0}$.

Definition 3.4 (holomorphic germs) A holomorphic germ at a point $s_{0} \in \mathbb{C}^{p}$ of distributions on $X$ is an equivalence class of holomorphic families of distributions on $X$ w.r.t. the natural equivalence relation: $(t(s))_{s \in \Omega_{1}} \sim(u(s))_{s \in \Omega_{2}}$ if there exists $\Omega_{3} \subset \Omega_{1} \cap \Omega_{2}$ such that $s_{0} \in \Omega_{3}$ and $t(s)=u(s)$ for all $s \in \Omega_{3}$. This set is denoted by $\mathcal{D}^{\prime}\left(X, \mathcal{O}_{s_{0}}\left(\mathbb{C}^{p}\right)\right)$.

Example 3.1 The family of distributions $t(s): \varphi \in C_{c}^{\infty}(\mathbb{R}) \mapsto \int_{\mathbb{R}} e^{s x} \varphi(x) d x$ defines a holomorphic germ of distributions at $s=0$ with real coefficients.

Meromorphic germs of distributions. Once we have a proper definition for holomorphic families of distributions, we can give a very natural definition of meromorphic families of distributions as follows :

Definition 3.5 (meromorphic family of distributions with linear poles) For a complex domain $\Omega \subset \mathbb{C}^{p}$, a meromorphic family of distributions on $\Omega$ is a holomorphic family $(t(s))_{s \in \Omega \backslash\left\{s ; L_{1}=\cdots=L_{k}(s)=0\right\}}$ of distributions, where $L_{1}, \ldots, L_{k}$ are linear functions on $\mathbb{C}^{p}$, such that

$$
t(s)=\left(L_{1}(s) \ldots L_{k}(s)\right)^{-1} h(s), \forall s \in \Omega \backslash\left\{s ; L_{1}=\cdots=L_{k}(s)=0\right\}
$$

where $(h(s))_{s \in \Omega} \in \mathcal{D}^{\prime}(X, \mathcal{O}(\Omega))$.

Now we localize the above definition to germs at $s_{0} \in \mathbb{C}^{p}$ :

Definition 3.6 (meromorphic germs of distributions) A meromorphic germ of distributions at $s_{0}$ with linear poles is an equivalence class of meromorphic families of distributions on some neighborhood $\Omega$ of $s_{0}$ with linear poles under the equivalence relation: $t(s)_{s \in \Omega_{1} \backslash Y_{1}} \sim t^{\prime}(s)_{s \in \Omega_{2} \backslash Y_{2}}$, where $Y_{i}=\left\{s ; L_{1}^{i}=\cdots=L_{k_{i}}^{i}(s)=0\right\}, i=1,2$ with linear functions $L_{j}^{i} j=1, \cdots, k_{i}, i=1,2$, if there exist a complex domain $\Omega_{3} \subset \Omega_{1} \cap \Omega_{2}$, and linear functions $L_{1}^{3}, \cdots L_{k_{3}}^{3}$ such that $s_{0} \in \Omega_{3}, s_{0} \in Y_{1} \cap Y_{2}$, $Y_{3}=\left\{s ; L_{1}^{i}=\cdots=L_{k_{i}}^{i}(s)=0\right\} \subset Y_{1}, Y_{3} \subset Y_{2}$, and $t(s)_{s \in \Omega_{3} \backslash Y_{3}}=t^{\prime}(s)_{s \in \Omega_{3} \backslash Y_{3}}$. The set of meromorphic germs of distribution with real coefficients will be denoted by $\mathcal{D}^{\prime}\left(X, \mathcal{M}_{s_{0}}\left(\mathbb{C}^{p}\right)\right)$.

It is simple to show that:

Proposition 3.1 The set $\mathcal{D}^{\prime}\left(X, \mathcal{O}_{s_{0}}\left(\mathbb{C}^{p}\right)\right)$ is a vector subspace of $\mathcal{D}^{\prime}\left(X, \mathcal{M}_{s_{0}}\left(\mathbb{C}^{p}\right)\right)$.

\subsection{Power expansions of holomorphic germs}

Let us state a convenient proposition about power series expansion of holomorphic families of distributions whose proof is given in the appendix.

Proposition 3.2 Let $X$ be some smooth manifold, $\Omega \subset \mathbb{C}^{p}$ and $(t(s))_{s \in \Omega} \in \mathcal{D}^{\prime}(X, \mathcal{O}(\Omega))$ be some holomorphic family of distributions. Then near every $s_{0} \in \Omega, t_{s}$ admits a power series expansion

$$
t(s)=\sum_{\alpha} \frac{\left(s-s_{0}\right)^{\alpha}}{\alpha !} t_{\alpha}
$$

where $\alpha=\left(\alpha_{1}, \ldots, \alpha_{n}\right) \in \mathbb{N}^{n}$ and $t_{\alpha}$ is a distribution in $\mathcal{D}^{\prime}(X)$ such that for all test function $\varphi$, $\sum_{\alpha} \frac{\left(s-s_{0}\right)^{\alpha}}{\alpha !} t_{\alpha}(\varphi)$ converges as power series near $s_{0}$.

This classical result is just a multivariable version of [35, Theorem 1] which is stated for general locally convex spaces $E$, we include a proof in the appendix to make our text self-contained. 


\subsection{From Green functions to the heat kernel.}

The fundamental tool we use to investigate the singularities of Feynman amplitudes is the heat kernel. In this section, we recall its main properties and explain how one can express the regularized Green functions and the Feynman amplitudes in terms of the heat kernel.

\subsubsection{Heat kernels}

The complex powers of $P=-\Delta_{g}+V$ are related to the heat kernel $e^{-t P}$ in the following way (see also [34, paragraph 1.12 .14 p. 112]):

Proposition 3.3 Let $(M, g)$ be a smooth compact, connected Riemannian manifold without boundary and let $P=-\Delta_{g}+V, V \in C_{\geqslant 0}^{\infty}(M)$ or $M=\mathbb{R}^{d}$ with constant metric $g$ and $P=-\Delta_{g}+m^{2}, m \in \mathbb{R}_{\geqslant 0}$. Set $\Pi$ to be the spectral projector on $\operatorname{ker}(P)$, and $s \in \mathbb{C}$ with $\operatorname{Re}(s)>0$ then

$$
P^{-s}=\frac{1}{\Gamma(s)} \int_{0}^{\infty}\left(e^{-t P}-\Pi\right) t^{s} \frac{d t}{t}
$$

in the sense of bounded operator from $L^{2}(M, \mathbb{C}) \mapsto L^{2}(M, \mathbb{C})$ where $\Gamma$ is the Euler Gamma function. In the sense of Schwartz kernels :

$$
\mathfrak{G}^{s}(x, y)=\frac{1}{\Gamma(s)} \int_{0}^{\infty}\left(K_{t}(x, y)-\Pi(x, y)\right) t^{s} \frac{d t}{t}
$$

where $K_{t} \in C^{\infty}((0, \infty) \times M \times M)$ is the heat kernel.

Note that when $0 \notin \operatorname{ker}(P)$ and $M$ compact or when $M=\mathbb{R}^{d}$, then we can set $\Pi=0$.

Proof — The proposition is clear when $M=\mathbb{R}^{d}$ hence we just discuss the compact case. As a consequence of the compactness of $M$ and the fact that $P$ is an elliptic, positive, self-adjoint operator, $P$ has discrete spectrum denoted by $\sigma(P)$, the eigenfunctions $\left(e_{\lambda}\right)_{\lambda \in \sigma(P)}$ of $P$ form an orthonormal basis of $L^{2}(M, \mathbb{C})$, so for any $u \in L^{2}(M, \mathbb{C}), u=\sum\left\langle u, e_{\lambda}\right\rangle e_{\lambda}$. By definition, $P^{-s} u=\sum_{\lambda \in \sigma(P), \lambda \neq 0} \lambda^{-s}\left\langle u, e_{\lambda}\right\rangle e_{\lambda}$ where the sum on the right hand side converges absolutely in $L^{2}(M, \mathbb{C})$. And the spectral projector $\Pi$ on $\operatorname{ker}(P)$ is simply $\Pi(u)=\sum_{\lambda=0}\left\langle u, e_{\lambda}\right\rangle e_{\lambda}$.

The heat operator $e^{-t P}$ is a strongly continuous semigroup acting on $L^{2}(M, \mathbb{C})$. For every $u \in$ $L^{2}(M, \mathbb{C})$ :

$$
\left(e^{-t P}-\Pi\right) u=\sum_{\lambda \in \sigma(P) \backslash 0} e^{-t \lambda}\left\langle u, e_{\lambda}\right\rangle e_{\lambda}
$$

where the sum on the r.h.s converges in $L^{2}(M, \mathbb{C})$.

Therefore for $\lambda>0$, by a change of variable in the $\Gamma$ function $\lambda^{-s}=\frac{1}{\Gamma(s)} \int_{0}^{\infty} e^{-t \lambda} t^{s} \frac{d t}{t}$, it follows that the identity $P^{-s}=\frac{1}{\Gamma(s)} \int_{0}^{\infty}\left(e^{-t P}-\Pi\right) t^{s} \frac{d t}{t}$ holds true in operator sense where the integral on the r.h.s converges in operator norm. Hence the same identity should hold true for the corresponding Schwartz kernels.

\subsection{Local asymptotic expansions of heat kernels}

We will use the following property of the heat kernel asymptotics [14, Thm 2.30] (see also [66, Thm 7.15]) :

Theorem 3.1 ( Minakshisundaram-Pleijel) Let $(M, g)$ be a compact Riemannian manifold without boundary, $\varepsilon$ is the injectivity radius of $M$ and $P=-\Delta_{g}+V, V \in C_{\geqslant 0}^{\infty}(M)$. For any cutoff function $\psi: \mathbb{R}_{+} \mapsto[0,1]$ such that $\psi(s)=1$ if $s \leqslant \varepsilon^{2} / 4$ and $\psi(s)=0$ if $s>4 \varepsilon^{2} / 9$. Let $K_{t}(x, y) \in C^{\infty}((0, \infty) \times M \times M)$ denote the heat kernel, then there exist smooth real valued functions 
$a_{k}(x, y) \in C^{\infty}(M \times M), k=0,1, \cdots$, with $a_{0}(x, y)=1$, such that for all $(n, p) \in \mathbb{N}^{2}$, and differential operator $P\left(x, D_{x}\right)$ of degree $m$, there exists a constant $C>0$ such that for all $t \in(0,1]$ :

$$
\sup _{(x, y) \in M^{2}}\left|P\left(x, D_{x}\right) \partial_{t}^{p}\left(K_{t}(x, y)-\sum_{k=0}^{n} \psi\left(\mathbf{d}^{2}\right) \frac{e^{-\frac{\mathbf{d}^{2}(x, y)}{4 t}}}{(4 \pi t)^{\frac{d}{2}}} a_{k}(x, y) t^{k}\right)\right| \leqslant C t^{n-\frac{d}{2}-\frac{m}{2}-p}
$$

where $\mathbf{d}(.,):. M \times M \mapsto \mathbb{R}_{\geqslant 0}$ is the Riemannian distance function.

Note that our statement differs from the statement in 66] from the fact that we use a cut-off function $\psi$ since outside some neighborhood of the diagonal $\Delta \subset M \times M, K_{t}$ vanishes at infinite order in $t$ when $t \rightarrow 0$ (see the proof of [66. Thm $7.15 \mathrm{p}$. 102]). In case $M=\mathbb{R}^{d}$ with constant metric $g$ and $P=-\Delta_{g}+m^{2}, m \in \mathbb{R}_{\geqslant 0}$, we have the well known exact formula :

$$
K_{t}(x, y)=\frac{1}{(4 \pi t)^{\frac{d}{2}}} \exp \left(-\frac{|x-y|_{g}^{2}}{4 t}-t m^{2}\right)=\frac{\exp \left(-\frac{|x-y|_{g}^{2}}{4 t}\right)}{(4 \pi t)^{\frac{d}{2}}} \sum_{k=0}^{\infty} \frac{(-1)^{k} t^{k} m^{2 k}}{k !}
$$

which already appeared in definition 2.3 .

\section{Reduction of regularized Feynman amplitudes}

Recall our aim was to prove analytic continuation of the regularized amplitude

$$
t_{G}=\prod_{e \in E(G)} \mathfrak{G}^{s_{e}}\left(x_{i(e)}, x_{j(e)}\right)
$$

in $\mathcal{D}^{\prime}\left(M^{V(G)}, \mathcal{M}_{s_{0}}\left(\mathbb{C}^{E(G)}\right)\right)$ where $s_{0}=\left(s_{e}=1\right)_{e \in E(G)}$ and $\mathfrak{G}^{s}$ is the Schwartz kernel of the complex power $P^{-s}$. The main goal of this section is to prove a technical Theorem 4.1 which allows us to reduce our main Theorem 2.1 to the proof of an analytic continuation Theorem for simpler analytic objects. These are some kind of Feynman amplitudes introduced in definition 4.3 corresponding to labelled Feynman graphs defined in definition 4.2 which are graphs whose edges are decorated by some integer. Intuitively, the amplitude of the labelled graph is obtained from the regularized amplitude $t_{G}(s)$ where we replace the heat kernels appearing in the formula for the Green function $\mathfrak{G}^{s}=\frac{1}{\Gamma(s)} \int_{0}^{\infty} t^{s-1}\left(e^{-t P}-\Pi\right) d t$, by the heat kernel asymptotic expansion. The integers decorating the edges exactly correspond to the heat coefficients in the heat kernel asymptotic expansion.

\subsection{Holomorphicity of Green's function}

The next Lemma discusses analytical properties of the regularized Green function of the Schrödinger operator $P$ which is elliptic since its leading part coincides with the Laplace operator, it is therefore automatically self-adjoint by the symmetry assumption [73, p. 35].

Lemma 4.1 Let $(M, g)$ be a smooth compact, connected Riemannian manifold without boundary and let $P=-\Delta_{g}+V, V \in C_{\geqslant 0}^{\infty}(M)$ or $M=\mathbb{R}^{d}$ with constant metric $g$ and $P=-\Delta_{g}+m^{2}, m \in \mathbb{R}_{\geqslant 0}$. Denote by $K_{t}$ the corresponding heat kernel. Then :

1. For all $k \in \mathbb{N}$, if $\operatorname{Re}(s)>\frac{d}{2}+k, \mathfrak{G}^{s}$ is a $C^{k}$ function on $M \times M$.

2. For all $k \in \mathbb{N}$, a compact subset $K \subset M \times M \backslash$ Diagonal, the kernel $\mathfrak{G}^{s}$ is holomorphic in $s$ and valued in $C^{k}(K)$.

3. If we write

$$
\mathfrak{G}^{s}(x, y)=\mathfrak{G}_{\leq}^{s}(x, y)+\mathfrak{G}_{\geq}^{s}(x, y)
$$


where

$$
\mathfrak{G}_{\geq}^{s}(x, y)=\frac{1}{\Gamma(s)}\left(\int_{0}^{1}\left(K_{t}-\Pi\right)(x, y) t^{s-1} d t\right), \mathfrak{G}_{\leq}^{s}(x, y)=\frac{1}{\Gamma(s)}\left(\int_{1}^{\infty}\left(K_{t}-\Pi\right)(x, y) t^{s-1} d t\right)
$$

then $\mathfrak{G}_{<}^{s}(x, y)$ is holomorphic in $s$ and valued in $C^{\infty}(M \times M)$ which is denoted by $\mathfrak{G}_{\leq}^{s} \in \bar{C}^{\infty}\left(M \times M, \mathcal{O}\left(\mathbb{C}^{p}\right)\right)$.

The proof of these classical properties, when $M$ is compact, is recalled in the appendix. For $M=\mathbb{R}^{d}$, they follow from straightforward computations.

\subsection{Reduction to local charts and localization near deepest diagonal.}

The purpose of the next two Lemmas is to localize the proof of our main Theorem about the analytic continuation of the distribution $t_{G}(s)$ to neighborhoods of the deepest diagonals in $M^{V(G)}$.

Lemma 4.2 Let $X$ be a manifold without boundary, $s_{0} \in \mathbb{C}^{p}$ then $t(s) \in \mathcal{D}^{\prime}\left(X, \mathcal{M}_{s_{0}}\left(\mathbb{C}^{p}\right)\right)$ iff for every $x \in N$, there exists a neighborhood $U_{x}$ of $x$ such that $\left.t(s)\right|_{U_{x}} \in \mathcal{D}^{\prime}\left(U_{x}, \mathcal{M}_{s_{0}}\left(\mathbb{C}^{p}\right)\right)$.

If $X$ is non compact, we require that there are linear functions $\left(L_{i}\right)_{i=1}^{k}$ corresponding to a fixed polar set $Y=\left\{s ; L_{1}(s)=\cdots=L_{k}(s)=0\right\} \subset \mathbb{C}^{p}, s_{0} \in Y$ such that $\left.t(s)\right|_{U_{x}} \in \mathcal{D}^{\prime}\left(U_{x}, \mathcal{M}_{s_{0}}\left(\mathbb{C}^{p}\right)\right)$ singular along the polar set $Y$.

Proof - The direct implication is straightforward. Assume that for every $x \in X$, there exists a neighborhood $U_{x}$ of $x$ such that $\left.t(s)\right|_{U_{x}} \in \mathcal{D}^{\prime}\left(U_{x}, \mathcal{M}_{s_{0}}\left(\mathbb{C}^{p}\right)\right)$. Then by local compactness, there is a locally finite subcover $\left(U_{i}\right)_{i}$ of $N$ such that $\left.t(s)\right|_{U_{i}} \in \mathcal{D}^{\prime}\left(U_{i}, \mathcal{M}_{s_{0}}\left(\mathbb{C}^{p}\right)\right)$. Let $\left(\chi_{i}\right)_{i}$ be a partition of unity where each $\chi_{i}$ is supported in $U_{i}$. Then for every test function $\varphi \in C_{c}^{\infty}(X),\langle t(s), \varphi\rangle=\sum_{i}\left\langle t(s), \chi_{i} \varphi\right\rangle$ is a finite sum of meromorphic germs with linear poles at $s_{0}$. In the non compact case, the polar set $Y$ is fixed. Therefore the sum is a meromorphic germ with linear poles at $s_{0}$. This is a finite sum, hence $s \mapsto\langle t(s), \varphi\rangle$ is meromorphic with linear poles at $s_{0}$ with linear poles.

The next Lemma is inspired by the seminal work of Popineau and Stora [60] and it states that it is enough to work our analytic continuation problem for the distributions $t_{G} \in \mathcal{D}^{\prime}\left(M^{V(G)}\right)$ near the deepest diagonals :

Lemma 4.3 (Popineau-Stora Lemma) If for any graph $G$, and any $x \in M$, there is some neighborhood $U_{x}$ of $x$, such that $\left.t_{G}\right|_{U_{x}^{V(G)}} \in \mathcal{D}^{\prime}\left(U_{x}^{V(G)}, \mathcal{M}_{s_{0}}\left(\mathbb{C}^{p}\right)\right)$ where $s_{0}=\left(s_{e}=1\right)_{e \in E(G)}$, then $t_{G} \in$ $\mathcal{D}^{\prime}\left(M^{V(G)}, \mathcal{M}_{s_{0}}\left(\mathbb{C}^{p}\right)\right), s_{0}=\left(s_{e}=1\right)_{e \in E(G)}$ for every graph $G$.

Proof - We prove it by induction on the number of vertices of the graph $G$. For $|V(G)|=2$, $t_{G}=\prod_{e=1}^{E} \mathfrak{G}^{s_{e}}(x, y)$. For a point $(x, y) \in M^{2}=M^{V(G)}$, if $x \neq y$, consider neighborhoods $V_{x}$ of $x$ in $M$ and $V_{y}$ of $y$ in $M$, such that $V_{x} \cap V_{y}=\emptyset$, then $\left\{V_{x} \times V_{y}\right\}_{(x, y) \in M^{2}, x \neq y} \bigcup\left\{U_{x} \times U_{x}\right\}_{x \in M}$ form an open cover of $M^{2}$, it has a locally finite subcover $\left\{W_{i}\right\}_{i}$ with $W_{i}=V_{x_{i}} \times V_{y_{i}}$ or $U_{x_{i}} \times U_{x_{i}}$. Let $\left\{\chi_{i}\right\}_{i}$ be a partition of unity supported on $\left\{W_{i}\right\}_{i}$, then $t_{G}=\sum_{i} t_{G} \chi_{i}$, where each $t_{G} \chi_{i}$ is holomorphic at $s_{0}$ if the support of $\chi_{i}$ does not intersect the diagonal by Lemma 4.1 or has meromorphic continuation at $s_{0}$ by assumption. Now the claim follows from Lemma 4.2 applied to the manifold $X=M^{V(G)}$.

Now $|V(G)|=n>2$ and assume the result holds for all graphs whose number of vertices are strictly less than $n$. Denote by $d_{n}=\left\{x_{1}=\cdots=x_{n}\right\} \subset M^{n}$, the deepest diagonal in the configuration space $M^{n}$. For $\left(x_{1}, \ldots, x_{n}\right) \in M^{n} \backslash d_{n}$, let $I=\left\{i \mid x_{i}=x_{1}\right\}$, and $I^{c}=\{1, \ldots, n\} \backslash I$, then $I \neq \emptyset$, $I^{c} \neq \emptyset$, and for any $j \in I^{c}$ there are neighborhoods $U_{j}$ of $x_{1}$ and $V_{j}$ of $x_{j}$ such that $U_{j} \cap V_{j}=\emptyset$. Let $\mathcal{V}=\left(\cap_{j \in I^{c}} U_{j}\right)^{|I|} \times \prod_{j \in I^{c}} V_{j}$, then $\mathcal{V} \subset M^{n} \backslash d_{n}$, and $x_{i} \neq x_{j}, \forall(i, j) \in I \times I^{c}$ for all $\left(x_{1}, \ldots, x_{n}\right) \in \mathcal{V}$. Then we partition the set of edges $E(G)$ of the graph $G$ in three parts : $E(G)=E_{I} \cup E_{I^{c}} \cup E_{I I^{c}}$, where $E_{I}$ (resp $E_{I^{c}}$ ) is the set of edges of $G$ whose incident vertices are in $I$ (resp $I^{c}$ ) i.e. every edge $e \in E_{I}$ (resp $e \in E_{I^{c}}$ ) is bounded by vertices in $I$ (resp $\left.I^{c}\right)$. The remaining subset of edges is 
denoted by $E_{I I^{c}}$ and is made of all edges $e \in E(G)$ which are neither in $E_{I}$ nor in $E_{I^{c}}$. By that, we mean the edges of $E_{I I^{c}}$ only connect some vertex in $I$ with another vertex of $I^{c}$. So we write $\left(x_{1}, \ldots, x_{n}\right)=\left(x_{i}, x_{j}\right)_{i \in I, j \in I^{c}} \in M^{I} \times M^{I^{c}}$. Similarly the complex variables $\left(s_{e}\right)_{e \in E(G)}$ attached to the edges of $G$ will be divided in three groups corresponding to the edges $E_{I}, E_{I^{c}}$ and $E_{I I^{c}}$ respectively. Then we decompose the amplitude $t_{G}$ as a product of three factors :

$$
\begin{aligned}
t_{G}\left(\left(s_{e}\right)_{e \in E(G)} ;\left(x_{i}, x_{j}\right)_{i \in I, j \in I^{c}}\right) & =t_{I}\left(\left(s_{e}\right)_{e \in E_{I}} ;\left(x_{i}\right)_{i \in I}\right) t_{I^{c}}\left(\left(\left(s_{e}\right)_{e \in E_{I^{c}}} ;\left(x_{j}\right)_{j \in I^{c}}\right)\right. \\
& \times t_{I I^{c}}\left(\left(s_{e}\right)_{e \in E_{I I^{c}}} ;\left(x_{i}, x_{j}\right)_{i \in I, j \in I^{c}}\right)
\end{aligned}
$$

where $t_{I}=\prod_{e \in E_{I}} \mathfrak{G}^{s_{e}}, t_{I^{c}}=\prod_{e \in E_{I^{c}}} \mathfrak{G}^{s_{e}}, t_{I I^{c}}=\prod_{e \in E_{I I^{c}}} \mathfrak{G}^{s_{e}}$.

By the induction assumption, both $t_{I}$ and $t_{I^{c}}$ are distributions in $\mathcal{D}^{\prime}\left(M^{I}, \mathcal{M}_{s_{0 I}}\left(\mathbb{C}^{E_{I}}\right)\right), s_{0 I}=\left(s_{e}=\right.$ 1) $)_{e \in E_{I}}$ and $\mathcal{D}^{\prime}\left(M^{I^{c}}, \mathcal{M}_{s_{0 I^{c}}}\left(\mathbb{C}^{E_{I^{c}}}\right)\right), s_{0 I^{c}}=\left(s_{e}=1\right)_{e \in E_{I^{c}}}$ respectively. Then by Lemma 7.1, the exterior product $t_{I}\left(\left(s_{e}\right)_{e \in E_{I}} ;\left(x_{i}\right)_{i \in I}\right) t_{I^{c}}\left(\left(\left(s_{e}\right)_{e \in E_{I^{c}}} ;\left(x_{j}\right)_{j \in I^{c}}\right)\right.$ of distributions depending on different variables is an element in $\mathcal{D}^{\prime}\left(M^{n}, \mathcal{M}_{s_{0 I I^{c}}}\left(\mathbb{C}^{E_{I} \cup E_{I^{c}}}\right)\right), s_{0 I^{I^{c}}}=\left(s_{e}=1\right)_{e \in E_{I} \cup E_{I^{c}}}$. Now the factor $t_{I I^{c}}$ contains only product of propagators $\mathfrak{G}^{s_{e}}\left(x_{i}, x_{j}\right)$ where $x_{i} \neq x_{j}$, so in the open subset $\mathcal{V} \subset M^{n}$, $\mathfrak{G}^{s_{e}}\left(x_{i}, x_{j}\right) \in C^{\infty}(M \times M \backslash$ Diagonal, $\mathcal{O}(\mathbb{C}))$ in the variables $\left(x_{i}, x_{j}\right)$ by Lemma 4.1. Thus on $\mathcal{V}$, $t_{I I^{c}} \in C^{\infty}\left(\mathcal{V}, \mathcal{O}\left(\mathbb{C}^{E_{I I^{c}}}\right)\right)$ which means $t_{I I^{c}}$ is holomorphic in the parameter $\left(s_{e}\right)_{e \in E_{I I^{c}}} \in \mathbb{C}^{E_{I I^{c}}}$. We conclude that near any element of $M^{n}$, there is some open neighborhood $U \subset M^{n}$ such that $t_{G} \in \mathcal{D}^{\prime}\left(U, \mathcal{M}_{s_{0}}\left(\mathbb{C}^{E(G)}\right)\right), s_{0}=\left(s_{e}=1\right)_{e \in E(G)}$. Then by Lemma $4.2, t_{G} \in \mathcal{D}^{\prime}\left(M^{n}, \mathcal{M}_{s_{0}}\left(\mathbb{C}^{E(G)}\right)\right), s_{0}=$ $\left(s_{e}=1\right)_{e \in E(G)}$.

\subsection{Reductions to integrals on cubes.}

In the representation of the Green function as integral of the heat kernel over $(0,+\infty)$, we would like to get rid of the low energy part which is $\mathfrak{G}_{\leqslant}^{s}=\int_{1}^{\infty} d t\left(K_{t}-\Pi\right) t^{s-1}$ which is smooth and holomorphic in $s$ so it does not contribute to the singularities of $t_{G}$. We thus reduce the study of $t_{G}$, to the study of some formula $P_{G}$ which contains only integrals over cubes which are easier to handle and contain all the singularities of $t_{G}$.

Definition 4.1 For a graph $G$, and $E \subset E(G)$, the subgraph induced by $E$ is the subgraph $H$ of $G$, such that $E(H)=E, V(H)=\{v \in V(G) \mid v$ is a vertex incident to some $e \in E\}$.

Proposition 4.1 If for every graph $G$, the product

$$
P_{G}(s)=\prod_{e \in E(G)} \frac{1}{\Gamma\left(s_{e}\right)}\left(\int_{0}^{1}\left(K_{\ell_{e}}-\Pi\right)\left(x_{i(e)}, x_{j(e)}\right) \ell_{e}^{s_{e}-1} d \ell_{e}\right)
$$

extends to $\mathcal{D}^{\prime}\left(M^{V(G)}, \mathcal{M}_{s_{0}}\left(\mathbb{C}^{E(G)}\right)\right)$ at $s_{0}=\left(s_{e}=1\right)_{e \in E(G)}$, then $t_{G}(s)$ extends to $\mathcal{D}^{\prime}\left(M^{V(G)}, \mathcal{M}_{s_{0}}\left(\mathbb{C}^{E(G)}\right)\right)$ at $s_{0}=\left(s_{e}=1\right)_{e \in E(G)}$.

Proof — For all $R e\left(s_{e}\right)>\frac{d}{2}$, since $\mathfrak{G}^{s}, \mathfrak{G}_{\geq}^{s}, \mathfrak{G}_{\leq}^{s}$ all belong to $C^{0}(M \times M)$ by Lemma 4.1 the following product makes perfect sense :

$$
t_{G}(s)=\prod_{e \in E(G)}\left(\mathfrak{G}_{\leq}^{s_{e}}+\mathfrak{G}_{\geq}^{s_{e}}\right)=\prod_{e \in E(G)} \mathfrak{G}_{\geq}^{s_{e}}+\prod_{e \in E(G)} \mathfrak{G}_{\leq}^{s_{e}}+\sum_{E_{1} \cup E_{2}=E(G)}\left(\prod_{e \in E_{1}} \mathfrak{G}_{\geq}^{s_{e}}\right)\left(\prod_{e \in E_{2}} \mathfrak{G}_{\leq}^{s_{e}}\right)
$$

where the sum runs over all partitions $E(G)=E_{1} \cup E_{2}, E_{1} \neq \emptyset, E_{2} \neq \emptyset$. Therefore :

$$
t_{G}(s)=P_{G}(s)+\underbrace{\prod_{e \in E(G)} \mathfrak{G}_{\leq}^{s_{e}}}+\sum_{E_{1} \cup E_{2}=E(G)} P_{G\left(E_{1}\right)}(s) \underbrace{\left(\prod_{e \in E_{2}} \mathfrak{G}_{\leq}^{s_{e}}\right)}
$$


where $G\left(E_{1}\right)$ is the induced subgraph of $G$ by the subset $E_{1}$. The terms underbraced are in $C^{\infty}$ functions depending holomorphically on the parameters $s \in \mathbb{C}^{E(G)}$ near $\left(s_{e}=1\right)_{e \in E(G)}$ since each $\mathfrak{G}_{\leq}^{s} \in C^{\infty}(M \times M, \mathcal{O}(\mathbb{C}))$. By assumption, for all induced subgraph $G\left(E_{1}\right) \subset G, P_{G\left(E_{1}\right)}$ extends to $\mathcal{D}^{\prime}\left(M^{V\left(G\left(E_{1}\right)\right)}, \mathcal{M}_{s_{0}}\left(\mathbb{C}^{E_{1}}\right)\right), s_{0}=\left(s_{e}=1\right)_{e \in E_{1}}$. Therefore by Lemma 7.3 each product $P_{G\left(E_{1}\right)}(s)\left(\prod_{e \in E_{2}} \mathfrak{G}_{\leq}^{s_{e}}\right)$ has analytic continuation in $\mathcal{D}^{\prime}\left(M^{V(G)}, \mathcal{M}_{s_{0}}\left(\mathbb{C}^{E(G)}\right)\right), s_{0}=\left(s_{e}=1\right)_{e \in E(G)}$.

Therefore it is sufficient to study :

$$
P_{G}(s)=\prod_{e \in E(G)} \frac{1}{\Gamma\left(s_{e}\right)}\left(\int_{0}^{1}\left(K_{\ell_{e}}-\Pi\right)\left(x_{i(e)}, x_{j(e)}\right) \ell_{e}^{s_{e}-1} d \ell_{e}\right)
$$

Lemma 4.4 Let $G$ be a graph with $E$ edges and

$$
P_{G}(s)=\prod_{e \in E(G)} \frac{1}{\Gamma\left(s_{e}\right)}\left(\int_{[0,1]^{E(G)}} \prod_{e \in E(G)}\left(K_{\ell_{e}}-\Pi\right)\left(x_{i(e)}, x_{j(e)}\right) \ell_{e}^{s_{e}-1} d \ell_{e}\right) .
$$

If for all $e \in\{1, \ldots, E\}, \operatorname{Re}\left(s_{e}\right)>\frac{d}{2}$, then the integral defining $P_{G}(s)$ converges absolutely in $[0,1]^{E}$ uniformly in $\left(x_{1}, \ldots, x_{|V(G)|}\right) \in M^{V(G)}$.

Proof - First when $M=\mathbb{R}^{d}$ or if $M$ is compact and $0 \notin \operatorname{ker}(P)$ then $\Pi=0$. Otherwise, if $0 \in \operatorname{ker}(P)$, then the Schwartz kernel of $\Pi$ must a constant function (see appendix 7.3). Therefore, it is sufficient that $\operatorname{Re}(s)>0$ so that $\int_{0}^{1} \Pi(x, y) \ell^{s-1} d \ell$ is Riemann integrable. Now by Theorem 3.1 there exists a constant $C_{0}>0$ such that for $\ell \in(0,1]$ and for all $(x, y) \in M^{2}$ :

$$
\left|\left(K_{\ell}(x, y)-\frac{1}{(4 \pi \ell)^{\frac{d}{2}}} \psi\left(\mathbf{d}^{2}(x, y)\right) e^{-\frac{\mathbf{d}^{2}(x, y)}{4 \ell}} \sum_{0 \leqslant k \leqslant \frac{d}{2}+1} a_{k}(x, y) \ell^{k}\right)\right| \leqslant C_{0} .
$$

So by the triangular inequality and by positivity of the heat kernel, we have the bound

$$
0 \leqslant K_{\ell}(x, y) \leqslant \frac{1}{(4 \pi \ell)^{\frac{d}{2}}} \psi\left(\mathbf{d}^{2}(x, y)\right) e^{-\frac{\mathbf{d}^{2}(x, y)}{4 \ell}} \sum_{0 \leqslant k \leqslant \frac{d}{2}+1}\left|a_{k}(x, y)\right| \ell^{k}+C_{0} .
$$

From which we can bound the integral :

$$
\begin{aligned}
\int_{0}^{1} K_{\ell}(x, y)\left|\ell^{s-1}\right| d \ell & \leqslant \int_{0}^{1} \frac{1}{(4 \pi)^{\frac{d}{2}}} \psi\left(\mathbf{d}^{2}(x, y)\right) e^{-\frac{\mathbf{d}^{2}(x, y)}{4 \ell}} \sum_{0 \leqslant k \leqslant \frac{d}{2}+1}\left|a_{k}(x, y) \ell^{k+s-\frac{d}{2}-1}\right|+C_{0}\left|\ell^{s-1}\right| d \ell \\
& \leqslant \int_{0}^{1} \frac{1}{(4 \pi)^{\frac{d}{2}}} \sum_{0 \leqslant k \leqslant \frac{d}{2}+1}\left|a_{k}(x, y) \ell^{k+s-\frac{d}{2}-1}\right|+C_{0}\left|\ell^{s-1}\right| d \ell
\end{aligned}
$$

since $\psi e^{-\frac{\mathrm{d}^{2}}{4 \ell}} \leqslant 1$ and the right hand side is absolutely integrable when $\operatorname{Re}(s)>\frac{d}{2}$. Therefore

$$
\left(\int_{[0,1]^{E(G)}} \prod_{e \in E(G)}\left(K_{\ell_{e}}-\Pi\right)\left(x_{i(e)}, x_{j(e)}\right) \ell_{e}^{s_{e}-1} d \ell_{e}\right)=\prod_{e \in E(G)} \int_{0}^{1}\left(K_{\ell_{e}}-\Pi\right)\left(x_{i(e)}, x_{j(e)}\right) \ell_{e}^{s_{e}-1} d \ell_{e}
$$

is a product of convergent Riemann integrals, the above integral inversions make sense by Fubini which yields the claim of our Lemma. 
Now we set :

$$
I_{G}(s)=\prod_{e \in E(G)} \frac{1}{\Gamma\left(s_{e}\right)} \int_{0}^{1} K_{\ell_{e}}\left(x_{i(e)}, x_{j(e)}\right) \ell^{s_{e}-1} d \ell_{e}
$$

which is well-defined as soon as $\operatorname{Re}\left(s_{e}\right)>\frac{d}{2}, \forall e \in E(G)$ by the above arguments. Then

$$
P_{G}(s)=\sum_{E \subset E(G)} I_{G(E)}(s) \prod_{e \in E(G) \backslash E} \frac{\Pi\left(x_{i(e)}, x_{j(e)}\right)}{s_{e}}
$$

where $G(E)$ is the induced subgraph by the subset of edges $E \subset E(G)$. By the fact that $\int_{0}^{1} \Pi \ell^{s-1} d \ell=$ $\frac{\Pi}{s} \in C^{\infty}\left(M \times M, \mathcal{O}_{1}(\mathbb{C})\right)$, which is holomorphic near $s=1$, the products of spectral projectors do not contribute to the poles. So we can further reduce our study to the analytic continuation of $I_{G}(s)$.

\subsection{Distributional order}

In this step, we introduce a further reduction by replacing each $K_{\ell}$ in the integral formula of $I_{G}(s)$ by its heat asymptotic expansion and try to control the remainders.

$$
\frac{1}{\Gamma(s)} \int_{0}^{1} K_{\ell} \ell^{s-1} d \ell=\frac{1}{\Gamma(s)} \int_{0}^{1} \frac{e^{-\frac{\mathbf{d}^{2}(x, y)}{4 \ell}}}{(4 \pi \ell)^{\frac{d}{2}}}\left(\sum_{k=0}^{p} a_{k}(x, y) \psi\left(\mathbf{d}^{2}(x, y)\right) \ell^{k}\right)+h_{p}(\ell, x, y) \ell^{s-1} d \ell
$$

where $h_{p}(\ell, x, y)$ is the remainder in the heat asymptotics which satisfies the estimate $\left\|h_{p}\right\|_{m} \leqslant$ $C \ell^{p-\frac{d}{2}-\frac{m}{2}}$ by Theorem 3.1 and $\psi$ is the cut-off function from Theorem 3.1

We first introduce some refinement of Feynman graphs to keep track of the information on the heat coefficients for every edge. So these are basically Feynman graphs whose edges are decorated by integers which correspond to heat coefficients.

Definition 4.2 (labelled graph) For a set $S$, an S-labelled graph is a pair $(G, \vec{k})$ where $\vec{k}$ is a map $E(G) \rightarrow S$. If $S$ is $\mathbb{N}$, we call it shortly labelled graph, and for $e \in E(G)$, we use the short notation $k_{e}$ to denote the element $\vec{k}(e) \in \mathbb{N}$. If $S=\mathbb{R}_{>0}$, then the map $E(G) \mapsto \mathbb{R}_{>0}$, called the length function, is denoted by $\ell$ and we call such pair $(G, \ell)$ a metric graph. If $\ell$ is injective, then $(G, \ell)$ is called strict metric graph.

We next define Feynman amplitudes attached to labelled graphs.

Definition 4.3 For every labelled graph $(G, \vec{k})$, we define the corresponding amplitude $I_{G, \vec{k}}(s)$ as follows :

$$
I_{G, \vec{k}}(s)=\prod_{e \in E(G)} \frac{1}{\Gamma\left(s_{e}\right)} \int_{[0,1]^{E}} \prod_{e \in E(G)}\left(\frac{e^{-\frac{\mathbf{d}^{2}}{4 \ell_{e}}}}{(4 \pi)^{\frac{d}{2}}} a_{k_{e}} \psi\left(\mathbf{d}^{2}\right)\right)\left(x_{i(e)}, x_{j(e)}\right) \ell_{e}^{k_{e}-\frac{d}{2}+s_{e}-1} d \ell_{e}
$$

which is well-defined and holomorphic in $\left(s_{e}\right)_{e \in E(G)} \in \mathbb{C}^{E(G)}$ on the domain $s_{e}>\frac{d}{2}, e \in E(G)$ by exactly the same proof as in Lemma 4.4 .

Proposition 4.2 If for every graph $G$, there is $m \in \mathbb{N}$ depending on $G$, such that for all labels $\vec{k} \in \mathbb{N}^{E(G)}$, for all $x \in M$, there is an open neighborhood $U_{x} \subset M$ of $x$ such that $I_{G,} \vec{k}(s)$ has analytic continuation in $\mathcal{D}^{\prime, m}\left(U_{x}^{V(G)}, \mathcal{M}_{s_{0}}\left(\mathbb{C}^{E(G)}\right)\right), s_{0}=\left(s_{e}=1\right)_{e \in E(G)}$, then for all $G, t_{G}(s)$ extends in $\mathcal{D}^{\prime}\left(M^{V(G)}, \mathcal{M}_{s_{0}}\left(\mathbb{C}^{E(G)}\right)\right), s_{0}=\left(s_{e}=1\right)_{e \in E(G)}$. 
Proof - Let $n=|V(G)|$ and $U=U_{x}$. By Lemma 4.3 which allows us to localize our analytic continuation proof near the deepest diagonal of $M^{n}$, we only need to prove that $t_{G}(s)$ extends as a meromorphic germ of distributions at $\left(s_{e}=1\right)_{e \in E(G)}$ on $U^{n}$. For a test function $\varphi$,

$$
\begin{aligned}
& \left\langle I_{G}(s), \varphi\right\rangle \\
= & \int_{U^{n}}\left(\prod_{e \in E(G)} \frac{1}{\Gamma\left(s_{e}\right)} \int_{0}^{1} K_{\ell_{e}} \ell_{e}^{s-1} d \ell_{e}\right) \varphi d v\left(x_{1}\right) \ldots d v\left(x_{n}\right) \\
= & \int_{U^{n}} \prod_{e \in E(G)}\left(\sum_{k_{e}=0}^{p} \frac{1}{\Gamma\left(s_{e}\right)} \int_{0}^{1} \frac{e^{-\frac{\mathbf{d}^{2}}{4 \ell_{e}}}}{(4 \pi)^{\frac{d}{2}}} a_{k_{e}} \psi\left(\mathbf{d}^{2}\right) \ell^{k_{e}+s_{e}-\frac{d}{2}-1} d \ell_{e}+\frac{1}{\Gamma\left(s_{e}\right)} \int_{0}^{1} h_{p} \ell_{e}^{s_{e}-1} d \ell_{e}\right) \varphi d v\left(x_{1}\right) \ldots d v\left(x_{n}\right) \\
= & \int_{U^{n}}\left(\sum_{E_{1} \cup E_{2}=E(G)} \prod_{e \in E_{1}}\left(\sum_{k_{e}=0}^{p} \frac{1}{\Gamma\left(s_{e}\right)} \int_{0}^{1} \frac{e^{-\frac{\mathbf{d}^{2}}{4 \ell_{e}}}}{(4 \pi)^{\frac{d}{2}}} a_{k_{e}} \psi\left(\mathbf{d}^{2}\right) \ell^{k_{e}+s_{e}-\frac{d}{2}-1} d \ell_{e}\right) \prod_{e \in E_{2}}\left(\frac{1}{\Gamma\left(s_{e}\right)} \int_{0}^{1} h_{p} \ell_{e}^{s_{e}-1} d \ell_{e}\right)\right) \\
\times & \varphi d v\left(x_{1}\right) \ldots d v\left(x_{n}\right)
\end{aligned}
$$

where the sum runs over partitions $E_{1} \cup E_{2}=E(G)$. Therefore we obtain

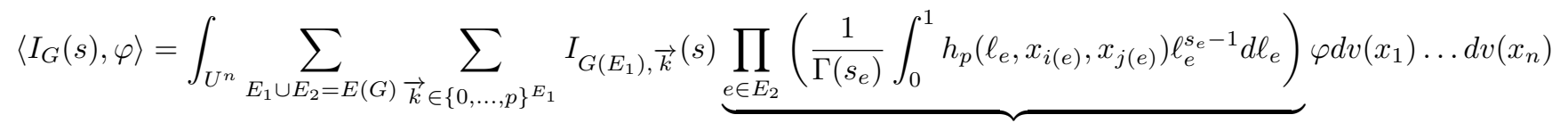

where the summation is over all $\vec{k} \in\{0,1, \cdots, p\}^{E(G)}$. By Theorem 3.1 we have the two estimates

$$
\left|D_{x}^{m} h_{p}\left(\ell_{e}, x, y\right) \ell_{e}^{s_{e}-1}\right| \leq C \ell^{p-\frac{d}{2}-\frac{m}{2}+s_{e}-1},
$$

and

$$
\left|D_{x}^{m} h_{p}\left(\ell_{e}, x, y\right) \ell_{e}^{s_{e}-1} \log \ell_{e}\right| \leq C \ell^{p-\frac{d}{2}-\frac{m}{2}+s_{e}-1+\varepsilon},
$$

for some $\varepsilon>0$. So when $p>\frac{d+m}{2}-1$, for every $e \in E_{2}$, there is a small neighborhood of $s_{e}=1$ such that the integral $\int_{0}^{1} h_{p}\left(\ell_{e}, x_{i(e)}, x_{j(e)}\right) \ell_{e}^{s_{e}-1} d \ell_{e}$ is absolutely convergent and depends holomorphically on $s_{e}$. Hence the term underbraced belongs to $C^{m}\left(U^{n}, \mathcal{O}_{s_{0}}\left(\mathbb{C}^{E_{2}}\right)\right), s_{0}=\left(s_{e}=1\right)_{e \in E_{2}}$, where $G\left(E_{2}\right)$ is the graph induced by $E_{2}$. Now we conclude the proof by noticing that the product of $I_{G\left(E_{1}\right), \vec{k}}(s) \in$ $\mathcal{D}^{\prime, m}\left(U^{n}, \mathcal{M}_{s_{0}}\left(\mathbb{C}^{E_{1}}\right)\right), s_{0}=\left(s_{e}=1\right)_{e \in E_{1}}$ with some element in $C^{m}\left(U^{n}, \mathcal{O}_{s_{0}}\left(\mathbb{C}^{E_{2}}\right)\right), s_{0}=\left(s_{e}=1\right)_{e \in E_{2}}$ yields an element of $\mathcal{D}^{\prime, m}\left(U^{n}, \mathcal{M}_{s_{0}}\left(\mathbb{C}^{E_{1} \cup E_{2}}\right)\right), s_{0}=\left(s_{e}=1\right)_{e \in E_{1} \cup E_{2}}$ by Lemma 7.3 proved in the appendix.

The next Theorem is the main result from the present section and summarizes all reduction steps performed above :

Theorem 4.1 (Reduction Theorem) Assume that for every graph $G$, there is an integer $m(G)$, such that for any $x \in M$, there is a chart $U_{x}$ of $M$ around $x$ such that for all $\vec{k}$, $\left.I_{G, \vec{k}}(s)\right|_{U_{x}^{n}}$ admits an analytic continuation in $\mathcal{D}^{\prime, m(G)}\left(U_{x}^{n}, \mathcal{M}_{s_{0}}\left(\mathbb{C}^{E(G)}\right)\right), s_{0}=\left(s_{e}=1\right)_{e \in E(G)}$. Then for a given graph $G$, let $m=\sup _{G^{\prime} \subset G} m\left(G^{\prime}\right)$, for any $p>\frac{d+m}{2}-1$, we have a decomposition

$$
t_{G}(s)=\sum_{G^{\prime} \subset G} \mathbf{m}_{G^{\prime}}(s) h_{G \backslash G^{\prime}}(s)
$$

where the sum runs over induced subgraphs $G^{\prime}$ of $G, \mathbf{m}_{G^{\prime}}(s)=\sum_{\vec{k} \in\{0, \ldots, p\} E\left(G^{\prime}\right)} I_{G^{\prime}, \vec{k}}(s) \in$

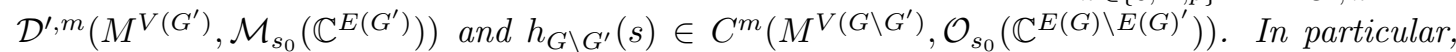
$t_{G}(s)$ extends as an element in $\mathcal{D}^{\prime}\left(M^{V(G)}, \mathcal{M}_{s_{0}}\left(\mathbb{C}^{E(G)}\right)\right), s_{0}=\left(s_{e}=1\right)_{e \in E(G)}$. 
So the above Theorem allows to reduce the proof of Theorem 2.1 to the analytic continuation of the simpler objects $I_{G, \vec{k}}(s)$ if we can control the distributional order of $I_{G, \vec{k}}(s)$ independently of the label $\vec{k} \in \mathbb{N}^{E(G)}$.

Proof - We use the following decomposition formula which summarizes the above three reduction steps, namely the reduction on cubes, the elimination of the spectral projector and the extraction of labelled graphs :

$$
\begin{aligned}
\left.t_{G}(s)\right|_{U_{x}^{n}} & =\sum_{E_{1} \cup E_{2} \cup E_{3} \cup E_{4}=E(G)}(\sum_{\vec{k} \in\{0, \ldots, p\}^{E_{1}}} \underbrace{I_{G\left(E_{1}\right), \vec{k}}(s)}) \prod_{e \in E_{2}}\left(\frac{1}{\Gamma\left(s_{e}\right)} \int_{0}^{1} h_{p}\left(\ell_{e}, x, y\right) \ell_{e}^{s_{e}-1} d \ell_{e}\right) \\
& \times \prod_{e \in E_{3}} \frac{\Pi\left(x_{i(e)}, y_{j(e)}\right)}{s_{e}} \prod_{e \in E_{4}} \mathfrak{G}_{\leq}^{s_{e}}
\end{aligned}
$$

where the sum runs over partitions $E_{1} \cup E_{2} \cup E_{3} \cup E_{4}=E(G)$. Then consider $m=\sup _{E_{1} \subset E(G)} m\left(G\left(E_{1}\right)\right)$ which is the supremum of distributional orders $m\left(G\left(E_{1}\right)\right)$ for $E_{1} \subset E(G), m$ is finite by assumption and bounds the distributional order of all the terms $I_{G\left(E_{1}\right), \vec{k}}(s)$ underbraced. Moreover, we saw in the proof of Proposition 4.2 that if we choose $p>\frac{d+m}{2}-1$, then the product

$$
\prod_{e \in E_{2}}\left(\frac{1}{\Gamma\left(s_{e}\right)} \int_{0}^{1} h_{p}\left(\ell_{e}, x, y\right) \ell_{e}^{s_{e}-1} d \ell_{e}\right) \prod_{e \in E_{3}} \frac{\Pi\left(x_{i(e)}, y_{j(e)}\right)}{s_{e}} \prod_{e \in E_{4}} \mathfrak{G}_{\leq}^{s_{e}}
$$

belongs to $C^{m}\left(M^{V\left(G\left(E_{2} \cup E_{3} \cup E_{4}\right)\right)}, \mathcal{O}_{s_{0}}\left(\mathbb{C}^{E_{2} \cup E_{3} \cup E_{4}}\right)\right), s_{0}=\left(s_{e}=1\right)_{e \in E_{2} \cup E_{3} \cup E_{4}}$. Therefore the whole product $t_{G}(s) \in \mathcal{D}^{\prime}\left(M^{V(G)}, \mathcal{M}_{s_{0}}\left(\mathbb{C}^{E(G)}\right)\right),\left(s_{e}=1\right)_{e \in E(G)}$. The above complicated formula can be written very concisely as

$$
t_{G}(s)=\sum_{G^{\prime} \subset G} \mathbf{m}_{G^{\prime}}(s) h_{G \backslash G^{\prime}}(s)
$$

where the sum runs over induced subgraphs $G^{\prime}$ of $G, \mathbf{m}_{G^{\prime}}(s)=\sum_{\vec{k} \in\{0, \ldots, p\}^{E\left(G^{\prime}\right)}} I_{G^{\prime}, \vec{k}}(s)$ and $h_{G \backslash G^{\prime}}=$ $\prod_{e \in E_{2}}\left(\frac{1}{\Gamma\left(s_{e}\right)} \int_{0}^{1} h_{p}\left(\ell_{e}, x, y\right) \ell_{e}^{s_{e}-1} d \ell_{e}\right) \prod_{e \in E_{3}} \frac{\Pi\left(x_{i(e)}, x_{j(e)}\right)}{s_{e}} \prod_{e \in E_{4}} \mathfrak{G}_{\leq}^{s_{e}}$ where $E_{2} \cup E_{3} \cup E_{4}$ forms a partition of $E(G) \backslash E\left(G^{\prime}\right)$.

\section{Desingularization of parameter space.}

Now that we reduced the proof of Theorem 2.1 to the proof of Theorem 4.1. we start by studying in local coordinates the amplitudes $I_{G, \vec{k}}(s) \in \mathcal{D}^{\prime}\left(M^{V(G)}\right)$ corresponding to labelled graphs $(G, \vec{k})$.

Fixing charts. For any $x \in M$, take a coordinate chart $U$ around $x$ such that $U \cong \mathbb{R}^{d}$ and $\bar{U} \subset M$ is compact and $\mathbf{d}\left(y_{1}, y_{2}\right)<\frac{\varepsilon}{2}$ for any $y_{1}, y_{2} \in U$. Since the volume form $d v$ on a Riemannian manifold reads $|\operatorname{det}(g)| d^{d} x$ in local coordinate chart, we may absorb the smooth function $|\operatorname{det}(g)|$ in the test function $\varphi$ and forget about the determinant of the metric in the local coordinate system. We number the vertices of $G$ by $\{1, \ldots, n\}$, and the edges by integers $\{1, \ldots, E\}$. For every edge $e$, we associate vertices $i(e), j(e)$ which are incident to $e$. Then for a test function $\varphi$ with $\operatorname{supp}(\varphi) \subset U^{n}$,

$$
\begin{aligned}
& \left\langle I_{G, \vec{k}}(s), \varphi\right\rangle \\
= & \frac{1}{(4 \pi)^{\frac{d E}{2}}} \prod_{e=1}^{E} \frac{1}{\Gamma\left(s_{e}\right)} \int_{[0,1]^{E}} d \ell_{1} \ldots d \ell_{E}\left(\int_{U^{n}} \prod_{e=1}^{E} \exp \left(-\frac{\mathbf{d}^{2}}{4 \ell_{e}}\right) \ell_{e}^{s_{e}+k_{e}-\frac{d}{2}-1} a_{k_{e}} \psi\left(\mathbf{d}^{2}\right) \tilde{\varphi} d^{d} x_{1} \ldots d^{d} x_{n}\right)
\end{aligned}
$$


where $\tilde{\varphi}=|\operatorname{det}(g)| \varphi$. This formula is well-defined when $\operatorname{Re}\left(s_{e}\right)>\frac{d}{2}, \forall e \in\{1, \ldots, E\}$ since the integration on $[0,1]^{E}$ is absolutely convergent, the integral on $U^{n}$ converges absolutely by compactness of the support of $\varphi$, hence we can integrate in order by Fubini Theorem. Furthermore, arguing as in the proof of Proposition 4.2 show that $\left\langle I_{G, \vec{k}}(s), \varphi\right\rangle$ is holomorphic in $s \in \mathbb{C}^{E}$ when $\operatorname{Re}\left(s_{e}\right)>\frac{d}{2}$.

By our choice of $U, \mathbf{d}^{2}$ is smooth on $U \times U$, it is enough to prove that :

$$
\frac{1}{(4 \pi)^{\frac{d E}{2}}} \int_{[0,1]^{E}} d \ell_{1} \ldots d \ell_{E}\left(\int_{\mathbb{R}^{d n}} \prod_{e=1}^{E} \exp \left(-\frac{\mathbf{d}^{2}}{4 \ell_{e}}\right) \ell_{e}^{s_{e}+k_{e}-\frac{d}{2}-1} a_{k_{e}} \psi\left(\mathbf{d}^{2}\right) \tilde{\varphi} d^{d} x_{1} \ldots d^{d} x_{n}\right)
$$

extends to a meromorphic germ of distribution at $\left(s_{e}=1\right)$. Note that this argument also applies to the case where $M=\mathbb{R}^{d}$ with constant metric $g$ and $P=-\Delta_{g}+m^{2}, m \in \mathbb{R}_{\geqslant 0}$.

\subsection{Smoothness problems and the need to resolve singularities.}

Assume we work on flat space $\mathbb{R}^{d}$, then to study the analytic continuation of $I_{G, \vec{k}}$, we need to study integrals of the form :

$$
\int_{[0,1]^{E}} d \ell_{1} \ldots d \ell_{E}\left(\int_{\mathbb{R}^{d n}} \prod_{e=1}^{E} \exp \left(-\frac{\left|x_{i(e)}-x_{j(e)}\right|^{2}}{4 \ell_{e}}\right) \ell_{e}^{s_{e}+k_{e}-\frac{d}{2}-1} a_{k_{e}} \psi\left(\mathbf{d}^{2}\right) \tilde{\varphi} d^{d} x_{1} \ldots d^{d} x_{n}\right) .
$$

The analytic continuation would come from integration by parts on the cube $[0,1]^{E}$ w.r.t. the variables $\left(\ell_{1}, \ldots, \ell_{E}\right)$. However, we see immediately that $e^{-|x-y|^{2} / 4 \ell}$ is not a smooth function of $(\ell, x, y) \in$ $[0,1] \times \mathbb{R}^{d} \times \mathbb{R}^{d}$. The problem occurs at the set $X=\{\ell=0, x-y=0\} \subset[0,1] \times \mathbb{R}^{d} \times \mathbb{R}^{d}$. A solution in global analysis is to consider the following smooth map :

$$
\pi:(t, x, h) \in[0,1] \times\left(\mathbb{R}^{d}\right)^{2} \mapsto(t, x, x+\sqrt{t} h) \in[0,1] \times\left(\mathbb{R}^{d}\right)^{2} .
$$

Note that after pull-back by $\pi$, we find that $e^{-|x-y|^{2} / 4 \ell} \circ \pi(x, t, h)=e^{-|h|^{2} / 4}$ which is now a smooth function near the preimage $\pi^{-1}(X)=\{t=0\}$ in $[0,1] \times\left(\mathbb{R}^{d}\right)^{2}$. We say that we resolved the singularities of $e^{-|x-y|^{2} / 4 \ell}$. For a discussion of why one needs to use blow-ups to study heat kernels and applications to index theory, the reader is referred to [52, p. 253]. Similarly, the product of exponentials $\prod_{e=1}^{E} \exp \left(-\frac{\left|x_{i(e)}-x_{j(e)}\right|^{2}}{4 \ell_{e}}\right)$ appearing in Feynman amplitudes is also not smooth on the whole domain of integration $\left(\ell_{1}, \ldots, \ell_{E}, x_{1}, \ldots, x_{n}\right) \in[0,1]^{E} \times \mathbb{R}^{d n}$ and integration by parts cannot be done. It follows that we must resolve the products $\prod_{e=1}^{E} \exp \left(-\frac{\left|x_{i(e)}-x_{j(e)}\right|^{2}}{4 \ell_{e}}\right)$ to make them smooth which is discussed in paragraph 5.4. Such resolution of singularities were studied by Speer on flat space building on the work of Hepp. Also, when $(M, g)$ is an analytic Riemannian manifold or when $M=\mathbb{R}^{d}$ with constant Euclidean metric, one can use Hironaka's resolution of singularities as in [5] or Bernstein-Sato polynomials to regularize Feynman amplitudes [24, 42. However, on a Riemannian manifold $(M, g)$, if for all $m \in M$, there is an open subset $U$ containing $m$ and a local coordinate system $\left(x_{1}, \ldots, x_{d}\right): U \subset M \mapsto \mathbb{R}^{d}$ such that for every $\left(m_{1}, m_{2}\right) \in U^{2}$, $\mathbf{d}\left(m_{1}, m_{2}\right)=\sqrt{\sum_{i=1}^{d}\left(x_{i}\left(m_{1}\right)-x_{i}\left(m_{2}\right)\right)^{2}}$ then $(M, g)$ is flat. Otherwise for generic Riemannian manifolds $(M, g)$, it is not possible to find good coordinates to make the distance function locally quadratic because of curvature. This makes our resolution of singularities more difficult to handle than the one appearing in the work of Speer and the fact that we work in the $C^{\infty}$ case and not in the analytic or algebraic category prevents us from using directly Hironaka's resolution of singularities or Bernstein-Sato polynomials. Following the tradition in QFT [36, 65, our strategy is essentially combinatorial and our blow-ups are encoded by spanning trees of Feynman graphs whose definition is recalled in the next paragraph. 


\subsection{Spanning trees of metric graphs.}

Let us first collect some definitions and classical results on graphs which are close to 48, paragraph 2.1]. Recall that for all graph we consider in the present paper, since we assume the graph has no self-loop, every edge $e$ is adjacent to two different vertices.

Definition 5.1 For a graph $G$,

- a path from vertex $u$ to $w$ in a graph $G$ is a sequence $\left(u=v_{1}, e_{1}, v_{2}, \cdots, v_{n}, e_{n}, v_{n+1}=w\right)$, where $v_{i} \in V(G), e_{j} \in E(G)$ such that the vertices bounding $e_{i}$ are $v_{i}$ and $v_{i+1}, u$ is the initial vertex and $w$ is the terminal vertex, $n$ is called the length. A path is simple if all the edges are distinct. If $u=w$, it is called a cycle.

- The set of subgraphs is ordered as follows, we say $G_{1} \subset G_{2}$ for two subgraphs $G_{1}, G_{2}$ of $G$ if $E\left(G_{1}\right) \subset E\left(G_{2}\right)$. A forest $T$ is a graph without any simple cycle and a tree is a connected forest.

- A spanning forest $T$ of a graph $G$ is a subgraph of $G$ which is a forest and is maximal for the inclusion relation among subgraphs which are forests. If $T$ is a tree, it is called spanning tree. For any graph $G$, we will often use the following equivalent characterizations of spanning forests which is a classical result in graph theory [50, p. 40-41]. A subgraph $T \subset G$ is a spanning forest if and only if $T$ is a forest whose complement contains $b_{1}(G)$ edges :

$$
b_{1}(G)=|E(G)|-|E(T)|
$$

where $b_{1}(G)$ is the first Betti number of $G$.

- To a metric graph $(G, \ell)$, a metric filtration of $G$ is an increasing family of subgraphs $G_{1} \subset G_{2} \subset$ $\cdots \subset G_{E}$ where $G_{i}$ is induced by the shortest $i$ edges where $E=|E(G)|$ is the number of edges in $G$. For a strict metric graph, the metric filtration is unique.

- For every forest $T \subset G$ and every subgraph $G_{i}$ of $G$, we define $\left.T\right|_{G_{i}}$ as the subgraph of $G_{i}$ induced by the subset of edges $E(T) \cap E\left(G_{i}\right) \subset E\left(G_{i}\right)$. We will call $\left.T\right|_{G_{i}}$ the trace of $T$ in $G_{i}$.

- If $T$ is a subgraph of $G$ induced by $E(T)$, and $e \in E(G) \backslash E(T)$ then we define $T \cup$ e as the subgraph of $G$ induced by $E(T) \cup e$. For every edge $e \in E(G)$, the graph $G \backslash e$ is the subgraph induced by $E(G) \backslash$ e.

Definition 5.2 For any permutation $\sigma \in S_{E}$ of $\{1, \ldots, E\}$, the simplex $\boldsymbol{\Delta}_{\sigma}$ :

$$
\Delta_{\sigma}=\left\{\ell_{\sigma(1)}<\cdots<\ell_{\sigma(E)}\right\}
$$

is called a sector of $[0,1]^{E}$.

Before we proceed, let us remark that for a graph $G$, an element $\ell \in[0,1]^{E(G)}$, which is a map $\ell: E(G) \rightarrow[0,1]$, naturally defines a metric graph $(G, \ell)$. To a strict metric graph $G$, the metric induces a natural strict ordering of edges by the length which defines an element $\ell \in[0,1]^{E(G)}$ in a unique sector. The next Theorem, due to Kruskal, aims to show how from a strict connected metric graph $(G, \ell)$, one can produce some algorithm which extracts a unique spanning tree $T$ in $G$.

Theorem 5.1 (Kruskal) For a connected strict metric graph $(G, \ell)$, let $G_{1} \subset \cdots \subset G_{E}=G$ be the unique metric filtration of $G$. We denote by $N_{i}$ the first Betti number $b_{1}\left(G_{i}\right)$ of $G_{i}$. Then there exists a unique spanning tree $T$ of $G$ such that for all $i \in\{1, \ldots, E\}$, its trace $\left.T\right|_{G_{i}}$ is a spanning forest of $G_{i}$.

Proof - Let $\ell: E(G) \rightarrow(0, \infty)$ be the length function. We shall assume that the edges $E(G)$ are numbered as $\left\{e_{1}, \ldots, e_{E}\right\}$ in such a way that $i<j \Longrightarrow \ell\left(e_{i}\right)<\ell\left(e_{j}\right)$. We construct the tree by the Kruskal algorithm [49] as described in [36, p. 107]. Notice that the requirement that $T$ is a tree 
implies that $T$ together with all traces $\left.T\right|_{G_{i}}$ contain no simple cycles. So $\left.T\right|_{G_{i}}$ is a forest and therefore its complement in $G_{i}$ contains at least $N_{i}$ edges of $G_{i}$. Also notice that for any graph $G$, for every $e \in E(G)$, we have the inequality $0 \leqslant b_{1}(G)-b_{1}(G \backslash e) \leqslant 1$. This implies that the sequence $N_{1}, N_{2}, \cdots$ is increasing.

Now we can construct the desired spanning tree $T$ : start from $G_{1}$ which has only one edge $\left\{e_{1}\right\}$ hence contains no simple cycle, $N_{1}=0$. Let us denote by $i_{1}$ the first integer such that $b_{1}\left(G_{i_{1}}\right)=1$, similarly define $\left\{i_{2}, \ldots, i_{N_{E}}\right\} \subset\{1, \ldots, E\}$ such that $b_{1}\left(G_{i_{2}}\right)=2, \ldots, b_{1}\left(G_{i_{N_{E}}}\right)=N_{E}=b_{1}(G)$ and every $i_{j}$ is the smallest integer so that $b_{1}\left(G_{i_{j}}\right)=j$ for any $j=1, \cdots, N_{E}$. Set $i_{0}=1$, then we have an increasing family of subgraphs $G_{i_{0}} \subset G_{i_{1}} \subset \cdots \subset G_{i_{N_{E}}} \subset G$. Let $G_{i}=G_{i-1} \cup e_{i}$ and we set $T=G \backslash \cup_{j=1}^{N_{E}} e_{i_{j}}$. We prove that the subgraph $T$ constructed above has the property that its trace $\left.T\right|_{G_{i}}$ to every subgraph $G_{i}$ is a spanning forest in $G_{i}$ by induction for $j=1, \ldots, E$. First, we initialize the induction for $j=1, G_{1}$ contains just one edge hence $\left.T\right|_{G_{1}}=G_{1}$ is a spanning tree in $G_{1}$. Assume that $\left.T\right|_{G_{k}}$ is a spanning forest in $G_{k}$, then there are two cases:

Case $1: b_{1}\left(G_{k}\right)=b_{1}\left(G_{k+1}\right)$ i.e. $N_{k}=N_{k+1}$ so $e_{k+1} \in T$, and $\left.T\right|_{G_{k+1}}=\left.T\right|_{G_{k}} \cup e_{k+1}$, and let us prove that $\left.T\right|_{G_{k}} \cup e_{k+1}$ is a spanning forest in $G_{k+1}$. First $\left.T\right|_{G_{k}} \cup e_{k+1}$ contains no simple cycle. Since if it contained a simple cycle $\gamma$, then $e_{k+1}$ would belong to $\gamma$ and therefore $\left.T\right|_{G_{k}}$ would be a spanning forest in $G_{k+1}$, so $b_{1}\left(G_{k+1}\right)=b_{1}\left(G_{k}\right)+1$ by equation 17 which contradicts $b_{1}\left(G_{k}\right)=b_{1}\left(G_{k+1}\right) .\left.T\right|_{G_{k}} \cup e_{k+1}$ is thus a forest. $\left.T\right|_{G_{k}}$ is spanning in $G_{k}$ hence $\left.T\right|_{G_{k}} \cup e_{k+1}$ meets all vertices of $G_{k+1}$ and is spanning in $G_{k+1}$.

Case $2: b_{1}\left(G_{k}\right)+1=b_{1}\left(G_{k+1}\right)$ and by definition $\left.T\right|_{G_{k+1}}=\left.\left.T\right|_{G_{k}} \cdot T\right|_{G_{k}}$ is obviously a forest in $G_{k+1}$, its complement in $G_{k+1}$ contains $b_{1}\left(G_{k}\right)+1=b_{1}\left(G_{k+1}\right)$ edges by construction which implies it is spanning by equation 17 .

Now we use induction to prove the uniqueness of $T$, in fact, we prove $\left.T\right|_{G_{k}}$ is unique for any $k$. The initial step is trivial, and in general there are two cases. Either $b_{1}\left(G_{k}\right)=b_{1}\left(G_{k+1}\right)$, then $\left.T\right|_{G_{k+1}}=\left.T\right|_{G_{k}} \cup e_{k+1}$ or $b_{1}\left(G_{k}\right)+1=b_{1}\left(G_{k+1}\right)$ then $\left.T\right|_{G_{k+1}}=\left.T\right|_{G_{k}}$, so our algorithm produces a unique spanning tree.

Corollary 5.1 Let $(G, \ell)$ be a strict metric graph and $T$ be the unique spanning forest in $T$ from Theorem 5.1. Then for every edge $e \in E(G) \backslash E(T)$, there is a unique simple cycle $\gamma_{e}$ in $T \cup e$, such that for any edge $e^{\prime} \in \gamma_{e} \backslash\{e\}, \ell(e)>\ell\left(e^{\prime}\right)$.

Proof - Since $T$ is a spanning tree, $e \in E(G) \backslash E(T)$, so there is a unique simple cycle $\gamma_{e}$ in $T \cup e$. By our construction, if $e=e_{i_{j}}$, then $\left.T\right|_{G_{i_{j}-1}}=\left.T\right|_{G_{i_{j}}}$, and $\left.T\right|_{G_{i_{j}-1}} \cup e_{i_{j}}$ contains only one simple cycle $\gamma_{e}$, so for any edge $e^{\prime} \in \gamma_{e}, e^{\prime} \neq e, \ell(e)>\ell\left(e^{\prime}\right)$.

\subsection{Approximation of the Riemannian distance in normal coordinates.}

For a smooth Riemannian manifold $(M, g)$, and any $x \in M, g(x)$ is an inner product in $T_{x} M$ which induces an isomorphism $g(x): T_{x} M \rightarrow T_{x}^{*} M$ and thus an inner product $g^{-1}(x)$ on $T_{x}^{*} M$ by $g^{-1}(x)\left(w_{1}, w_{2}\right)=g(x)\left(g^{-1}(x)\left(w_{1}\right), g^{-1}(x)\left(w_{2}\right)\right)$. This defines a smooth metric $g^{-1}$ on $T^{*} M$.

For every $x \in M$, we will use normal coordinates $\left(U, \phi, x^{\mu}\right)$ around $x$, without loss of generality $U$ is assumed to be geodesically convex. The use of normal coordinates will be crucial since it allows us to approximate the squared distance $\mathbf{d}^{2}(x, y)$ by $|x-y|^{2}$ in local coordinates in Lemma 5.2 In some other coordinate chart, this approximation might not be as good. On $\left(U, \phi, x^{\mu}\right)$, there are two metrics: the Riemannian metric $g$ and the Euclidean metric $h$ :

$$
h\left(\frac{\partial}{\partial x^{\mu}}, \frac{\partial}{\partial x^{\nu}}\right)=h_{\mu \nu}=\delta_{\mu \nu} .
$$

For this Euclidean metric, we will use $|x-y|$ to denote the induced distance. We recall that at the origin, we have the identity $g_{\mu \nu}(0)=h_{\mu \nu}=\delta_{\mu \nu}$. The following Lemma which dates back to Hadamard can be found in [27, Lemma 8.3 p. 90], [55, (A.3) p. 31], 64, (38) p. 171] : 
Lemma 5.1 (Hadamard) We denote by $\mathbf{d}$ the Riemannian distance and $\phi=\mathbf{d}^{2}$. Then there exists a neighborhood $U$ of the diagonal $\subset M \times M$, such that $\phi \in C^{\infty}(U)$ and is symmetric, that is $\phi(x, y)=$ $\phi(y, x), \phi$ vanishes along the diagonal at order 2 and $\phi$ satisfies the Hamilton-Jacobi equation :

$$
g^{-1}\left(d_{x} \phi(x, y), d_{x} \phi(x, y)\right)=4 \phi(x, y) \text {. }
$$

Next we state an important Lemma which gives informations on the jets of the function $\phi=\mathbf{d}^{2}$ along the diagonal in $M \times M$.

Lemma 5.2 For $x_{0} \in M$, if $(U, \phi)$ is a normal coordinate system around $x_{0}$ such that $\phi\left(x_{0}\right)=0$ and the square of Riemannian distance $\phi$ is smooth on $U \times U$, then on $U \times U$,

$$
\phi(x, y)-g_{\mu \nu}(x)\left(x^{\mu}-y^{\mu}\right)\left(x^{\nu}-y^{\nu}\right)
$$

vanishes along the diagonal at order 3.

The proof can be found in appendix 7.5

\subsection{Resolving singularities using spanning trees.}

Let $(G, \ell)$ be a connected strict metric graph with edge set $E(G)$ identified with the set of integers $\{1, \ldots, E\}$ in such a way that $0 \leqslant \ell_{1}<\cdots<\ell_{E} \leqslant 1$. This means that the metric graph $(G, \ell)$ lies in a fixed sector $\boldsymbol{\Delta}=\left\{0<\ell_{1}<\cdots<\ell_{E}<1\right\} \subset[0,1]^{E}, \overline{\boldsymbol{\Delta}}$ denotes its closure $\left\{0 \leqslant \ell_{1} \leqslant \cdots \leqslant \ell_{E} \leqslant 1\right\}$. It is associated with a unique spanning tree $T$ by Theorem 5.1 and the vertices of both graphs $G$ and $T$ are numbered by $\{1, \ldots, n\}$. For any $(i, j) \in\{1, \ldots, n\}^{2}$, we denote by $\overrightarrow{i j}$ the unique simple path in $T$ from $i$ to $j$.

The product $\prod_{e \in E(G)} e^{-\frac{\mathbf{d}^{2}\left(x_{i(e)}, x_{j(e)}\right)}{4 \ell_{e}}} \psi\left(\mathbf{d}^{2}\left(x_{i(e)}, x_{j(e)}\right)\right)$, where $\psi$ is the cut-off function from Theorem 3.1 is not smooth near the algebraic set $X=\cup_{e \in E(G)}\left\{\ell_{e}\left(x_{i(e)}-x_{j(e)}\right)=0\right\} \subset \mathbb{R}^{d n} \times \overline{\boldsymbol{\Delta}}$. What we do next is give a recipe to resolve the singularities of such products by some explicit map $\pi$ which is defined as follows :

Definition 5.3 In the above notations, the map from $\mathbb{R}^{d} \times\left(\mathbb{R}^{d}\right)^{E(T)} \times[0,1]^{E}$ to $\mathbb{R}^{d n} \times \overline{\boldsymbol{\Delta}}$ is :

$$
\pi: \underbrace{\left(x,\left(h_{e}\right)_{e \in E(T)},\left(t_{k}\right)_{k=1}^{E}\right)}_{\in \mathbb{R}^{d} \times\left(\mathbb{R}^{d}\right)^{E(T)} \times[0,1]^{E}} \longmapsto \underbrace{\left(\left(x+\sum_{e \in \overrightarrow{1 i}}\left(\prod_{j \geqslant e} t_{j}\right) h_{e}\right)_{i=1}^{n},\left(\prod_{k \geqslant e} t_{k}^{2}\right)_{e=1}^{E}\right)}_{\in \mathbb{R}^{d n} \times \overline{\boldsymbol{\Delta}}}
$$

where the sum runs over all edges e in the path $\overrightarrow{1 i}$. The map $\pi$ depends on spanning tree $T$ hence on the strict ordering of $E(G)$ induced by the metric $\ell$.

In the sequel, we shall denote elements of the target space $\mathbb{R}^{d n} \times \overline{\boldsymbol{\Delta}}$ by $\left(x_{i}, \ell_{e}\right)_{1 \leqslant i \leqslant n, 1 \leqslant e \leqslant E}$. We check that the map $\pi: \mathbb{R}^{d} \times\left(\mathbb{R}^{d}\right)^{E(T)} \times[0,1]^{E} \mapsto \mathbb{R}^{d n} \times \overline{\boldsymbol{\Delta}}$ is a diffeomorphism outside some subset of measure zero.

Proposition 5.1 The map $\pi$ is a smooth diffeomorphism from $\mathbb{R}^{d} \times \mathbb{R}^{d(n-1)} \times(0,1)^{E}$ to $\mathbb{R}^{d n} \times \Delta$.

Proof - It is one to one since we can explicitly invert $\pi$ as $t_{E}=\ell_{E}^{\frac{1}{2}}, t_{e}=\left(\frac{\ell_{e+1}}{\ell_{e}}\right)_{n}^{\frac{1}{2}}$ for $e \leqslant E-1$ and the linear map: $\left(x,\left(h_{e}\right)_{e \in E(T)}\right) \in \mathbb{R}^{d} \times \mathbb{R}^{d(n-1)} \mapsto\left(x_{i}=x+\sum_{e \in \overrightarrow{1 i}_{i}}\left(\prod_{j \geqslant e} t_{j}\right) h_{e}\right)_{i=1}^{n} \in \mathbb{R}^{d n}$ is invertible when $\left(t_{j}\right)_{j} \in(0,1)^{E}$. Then the diffeomorphism property follows from an explicit calculation of the differential of $\pi$ whose determinant does not vanish when $t \in(0,1)^{E}$. 
Finally we may state the main Theorem from this section :

Theorem 5.2 (Resolution of singularities.) Let $g$ be a Riemannian metric on $\mathbb{R}^{d}$ and $\mathbf{d}: \mathbb{R}^{d} \times$ $\mathbb{R}^{d} \mapsto \mathbb{R}_{\geqslant 0}$ be the Riemannian distance whose injectivity radius is $\varepsilon$. Let $\pi$ be the map defined by equation 21. For any $\psi(t) \in C_{c}^{\infty}(\mathbb{R})$ such that $\psi(t)=1$ when $t \leqslant \frac{\varepsilon^{2}}{4}$ and $\psi(t)=0$ when $t>\frac{4 \varepsilon^{2}}{9}$, for every edge e $\in E(G)$ bounded by the vertices $(i(e), j(e))$, the pull-back:

$$
\pi^{*}\left(\psi\left(\mathbf{d}^{2}\left(x_{i(e)}, x_{j(e)}\right)\right) \frac{\mathbf{d}^{2}\left(x_{i(e)}, x_{j(e)}\right)}{\ell_{e}}\right)
$$

defines a smooth function in $\mathbb{R}^{d} \times \mathbb{R}^{d(n-1)} \times[0,1]^{E}$.

Proof - For $e \in E(T)$, set $x_{j(e)}-x_{i(e)}= \pm h_{e}$. Recall that $h_{e}$ has in fact $d$ components $\left(h_{e}^{\mu}\right)_{\mu=1}^{d}$. Then by Lemma 5.2 :

$$
\begin{aligned}
\pi^{*}\left(\frac{\mathbf{d}^{2}\left(x_{i(e)}, x_{j(e)}\right)}{\ell_{e}}\right) & =\frac{g_{\mu \nu}\left(x_{i(e)}\right) h_{e}^{\mu} h_{e}^{\nu}\left(\prod_{i \geqslant e} t_{i}\right)^{2}+R\left(\pi^{*} x_{i(e)}, \pi^{*} x_{i(e)} \pm\left(\prod_{i \geqslant e} t_{i}\right) h_{e}\right)}{\left(\prod_{i \geqslant e} t_{i}\right)^{2}} \\
& =g_{\mu \nu}\left(x_{i(e)}\right) h_{e}^{\mu} h_{e}^{\nu}+r_{e}(t, x, h)
\end{aligned}
$$

where

$$
r_{e}(t, x, h)=\frac{R\left(\pi^{*} x_{i(e)}, \pi^{*} x_{i(e)}+\left(\prod_{i \geqslant e} t_{i}\right) h_{e}\right)}{\left(\prod_{i \geqslant e} t_{i}\right)^{2}}=\frac{O\left(\left(\prod_{i \geqslant e} t_{i}\right)^{3}\left\|h_{e}\right\|^{3}\right)}{\left(\prod_{i \geqslant e} t_{i}\right)^{2}}=O\left(\left(\prod_{i \geqslant e} t_{i}\right)\left\|h_{e}\right\|^{3}\right)
$$

vanishes at order 3 in $\left(h_{e}\right)_{e \in T}$ and at order 1 in $\left(t_{e}\right)_{e=1}^{E}$ by Lemma 5.2 and $r_{e}$ is smooth.

If $e \notin E(T)$, then

$$
\pi^{*}\left(\frac{\mathbf{d}^{2}\left(x_{i(e)}, x_{j(e)}\right)}{\ell_{e}}\right)=\pi^{*}\left(\frac{g_{\mu \nu}\left(x_{i(e)}\right)\left(x_{i(e)}^{\mu}-x_{j(e)}^{\mu}\right)\left(x_{i(e)}^{\nu}-x_{j(e)}^{\nu}\right)}{\ell_{e}}\right)+\pi^{*}\left(\frac{R\left(x_{i(e)}, x_{j(e)}\right)}{\ell_{e}}\right) .
$$

where

$$
\begin{aligned}
& \pi^{*}\left(\frac{g_{\mu \nu}\left(x_{i(e)}\right)\left(x_{i(e)}^{\mu}-x_{j(e)}^{\mu}\right)\left(x_{i(e)}^{\nu}-x_{j(e)}^{\nu}\right)}{\ell_{e}}\right) \\
= & \frac{g_{\mu \nu}\left(x_{i(e)}\right)\left(\sum_{e^{\prime} \in \gamma_{e} \backslash e} \varepsilon\left(e^{\prime}\right)\left(\prod_{j \geqslant e^{\prime}} t_{j}\right) h_{e^{\prime}}^{\mu}\right)\left(\sum_{e^{\prime} \in \gamma_{e} \backslash e} \varepsilon\left(e^{\prime}\right)\left(\prod_{j \geqslant e^{\prime}} t_{j}\right) h_{e^{\prime}}^{\nu}\right)}{\left(\prod_{i \geqslant e} t_{i}\right)^{2}}
\end{aligned}
$$

where $\varepsilon\left(e^{\prime}\right)= \pm 1$ and $\gamma_{e}$ is the unique simple cycle in $T \cup e$ in Corollary 5.1. The important fact is that for all edge $e^{\prime}$ in the path $\gamma_{e} \backslash\{e\}, e^{\prime}<e$. It follows that:

$$
\begin{aligned}
& \pi^{*}\left(\frac{g_{\mu \nu}\left(x_{i(e)}\right)\left(x_{i(e)}^{\mu}-x_{j(e)}^{\mu}\right)\left(x_{i(e)}^{\nu}-x_{j(e)}^{\nu}\right)}{\ell_{e}}\right) \\
= & g_{\mu \nu}\left(x_{i(e)}\right)\left(\sum_{e^{\prime} \in \gamma_{e} \backslash e} \varepsilon\left(e^{\prime}\right)\left(\prod_{e^{\prime} \leqslant j<e} t_{j}\right) h_{e^{\prime}}^{\mu}\right)\left(\sum_{e^{\prime} \in \gamma_{e} \backslash e} \varepsilon\left(e^{\prime}\right)\left(\prod_{e^{\prime} \leqslant j<e} t_{j}\right) h_{e^{\prime}}^{\nu}\right)
\end{aligned}
$$

which is smooth since the product $\left(\pi_{i \geqslant e} t_{i}\right)^{2}$ on the denominator cancel out with the same powers appearing on the numerator. The same argument applies to the remainder term $\pi^{*}\left(\frac{R\left(x_{i(e)}, x_{j(e)}\right)}{\ell_{e}}\right)$. 


\subsection{Change of variables}

For a test function $\varphi$ supported in $\mathbb{R}^{d n}$, since the map $\pi$ is a smooth diffeomorphism from $\mathbb{R}^{d} \times$ $\mathbb{R}^{d(n-1)} \times(0,1)^{E}$ to $\mathbb{R}^{d n} \times \Delta$, we can take it as a change of variables for integration:

$$
\begin{aligned}
\left\langle I_{G, \vec{k}}(s), \varphi\right\rangle= & \frac{1}{(4 \pi)^{\frac{d E}{2}}}\left(\prod_{e=1}^{E} \frac{1}{\Gamma\left(s_{e}\right)}\right) \int_{[0,1]^{E}} d \ell_{1} \ldots d \ell_{E} \int_{\mathbb{R}^{d n}} \prod_{e=1}^{E} \exp \left(-\frac{\mathbf{d}^{2}\left(x_{i(e)}, x_{j(e)}\right)}{4 \ell_{e}}\right) \\
& \psi\left(\mathbf{d}^{2}\left(x_{i(e)}, x_{j(e)}\right)\right) \ell_{e}^{s_{e}+k_{e}-\frac{d}{2}-1} a_{k_{e}}\left(x_{i(e)}, x_{j(e)}\right) \tilde{\varphi} d^{d} x_{1} \ldots d^{d} x_{n} \\
= & \frac{1}{(4 \pi)^{\frac{d E}{2}}}\left(\prod_{e=1}^{E} \frac{1}{\Gamma\left(s_{e}\right)}\right) \sum_{\sigma \in S_{E}} \int_{\Delta_{\sigma}} d \ell_{1} \ldots d \ell_{E} \int_{\mathbb{R}^{d n}} \prod_{e=1}^{E} \exp \left(-\frac{\mathbf{d}^{2}\left(x_{i(e)}, x_{j(e)}\right)}{4 \ell_{e}}\right) \\
& \psi\left(\mathbf{d}^{2}\left(x_{i(e)}, x_{j(e)}\right)\right) \ell_{e}^{s_{e}+k_{e}-\frac{d}{2}-1} a_{k_{e}}\left(x_{i(e)}, x_{j(e)}\right) \tilde{\varphi} d^{d} x_{1} \ldots d^{d} x_{n}
\end{aligned}
$$

where $\sigma$ runs over the group $S_{E}$ of permutations of $\{1, \ldots, E\}$. The open simplices $\boldsymbol{\Delta}_{\sigma}$ do not cover the unit cube $[0,1]^{E}$. However the complement of $\cup_{\sigma} \boldsymbol{\Delta}_{\sigma}$ in $[0,1]^{E}$ has zero Lebesgue measure. Since for $\operatorname{Re}\left(s_{e}\right)_{e=1, \cdots, E}$ large enough, the integral $\int_{[0,1]^{E}} \prod_{e \in E(G)} \frac{e^{-\frac{\mathbf{d}^{2}}{4 \ell_{e}}}}{(4 \pi)^{\frac{d}{2}}} a_{k_{e}} \psi\left(\mathbf{d}^{2}\right) \ell_{e}^{k_{e}-\frac{d}{2}+s_{e}-1} d \ell_{e}$ is absolutely convergent and depends holomorphically in $s \in \mathbb{C}^{E}$, we have the equality of integrals

$$
\int_{[0,1]^{E}} \prod_{e \in E(G)} \frac{e^{-\frac{\mathbf{d}^{2}}{4 \ell_{e}}}}{(4 \pi)^{\frac{d}{2}}} a_{k_{e}} \psi\left(\mathbf{d}^{2}\right) \ell_{e}^{k_{e}-\frac{d}{2}+s_{e}-1} d \ell_{e}=\sum_{\sigma \in S(E)} \int_{\Delta_{\sigma}} \prod_{e \in E(G)} \frac{e^{-\frac{\mathbf{d}^{2}}{4 \ell_{e}}}}{(4 \pi)^{\frac{d}{2}}} a_{k_{e}} \psi\left(\mathbf{d}^{2}\right) \ell_{e}^{k_{e}-\frac{d}{2}+s_{e}-1} d \ell_{e},
$$

where both sides depend holomorphically on $\left(s_{e}\right)_{e}$ for $\operatorname{Re}\left(s_{e}\right)_{e=1, \cdots, E}$ large enough.

Now we can carry the change of variables in the fixed sector $\Delta=\left\{0<\ell_{1}<\cdots<\ell_{E}<1\right\}$ (the other terms will be obtained by permutation) which yields an expression of the form :

$$
\begin{aligned}
& \int_{\Delta} \prod_{e=1}^{E} \frac{d \ell_{e}}{\ell_{e}}\left(\int_{\left(\mathbb{R}^{d}\right)^{n}} \prod_{e=1}^{E} \exp \left(-\frac{\mathbf{d}^{2}\left(x_{i(e)}, x_{j(e)}\right)}{4 \ell_{e}}\right) \psi\left(\mathbf{d}^{2}\left(x_{i(e)}, x_{j(e)}\right)\right) \ell_{e}^{s_{e}+k_{e}-\frac{d}{2}} a_{k_{e}}\left(x_{i(e)}, x_{j(e)}\right) \tilde{\varphi} d^{d} x_{1} \ldots d^{d} x_{n}\right) \\
= & 2^{E} \int_{[0,1]^{E}} \prod_{e=1}^{E} \frac{d t_{e}}{t_{e}} \int_{\left(\mathbb{R}^{d}\right)^{n}} \pi^{*}\left(\prod_{e=1}^{E} \exp \left(-\frac{\mathbf{d}^{2}\left(x_{i(e)}, x_{j(e)}\right)}{4 \ell_{e}}\right) \psi\left(\mathbf{d}^{2}\left(x_{i(e)}, x_{j(e)}\right)\right)\right) \\
\times & \pi^{*}\left(\tilde{\varphi} \prod_{e \in E(G)} a_{k_{e}}\right)\left(\prod_{e \in E(G)} \ell_{e}^{\left(s_{e}+k_{e}\right)-\frac{d}{2}}\right)\left(\prod_{e \in E(T)} \ell_{e}^{\frac{d}{2}}\right) d^{d} x \prod_{e \in E(T)} d^{d} h_{e} .
\end{aligned}
$$

We can further simplify the product $\prod_{e \in E(G)} \ell_{e}(t)^{\left(s_{e}+k_{e}\right)-\frac{d}{2}} \prod_{e \in E(T)} \ell_{e}(t)^{\frac{d}{2}}$ as :

$$
\begin{aligned}
& \prod_{e \in E(G)}\left(\prod_{i \geqslant e} t_{i}\right)^{2\left(s_{e}+k_{e}\right)-d} \prod_{e \in E(T)}\left(\prod_{i \geqslant e} t_{i}\right)^{d}=\prod_{e \in E(T)}\left(\prod_{i \geqslant e} t_{i}\right)^{2 s_{e}+2 k_{e}} \prod_{e \notin E(T)}\left(\prod_{i \geqslant e} t_{i}\right)^{2 s_{e}-d+2 k_{e}} \\
= & \prod_{e \in E(G)}\left(\prod_{i \geqslant e} t_{i}\right)^{2 s_{e}+2 k_{e}} \prod_{e \notin E(T)}\left(\prod_{i \geqslant e} t_{i}\right)^{-d} \\
= & t_{E}^{2 s_{E}+2 k_{E}}\left(t_{E} t_{E-1}\right)^{2 s_{E-1}+2 k_{E-1}} \ldots\left(t_{E} \ldots t_{1}\right)^{2 s_{1}+2 k_{1}}\left(t_{E} \ldots t_{i_{k}}\right)^{-d} \ldots\left(t_{E} \ldots t_{i_{1}}\right)^{-d}
\end{aligned}
$$

where $\left(i_{1}<\cdots<i_{k}\right) \subset\{1, \ldots, E\}$ are the numbers decorating the edges in the complement of $E(T)$ and $k=b_{1}(G)$, hence :

$$
\prod_{e \in E(T)} \ell_{e}(t)^{2 s_{e}+2 k_{e}} \prod_{e \notin E(T)} \ell_{e}(t)^{2 s_{e}-d+2 k_{e}}=\prod_{e=1}^{E} t_{e}^{\sum_{i \leqslant e} 2 s_{i}+2 k_{i}-d b_{1}\left(G_{e}\right)}
$$


where $G_{e}$ denotes the graph induced by the first $e$ edges $\{1, \ldots, e\}$. This in turns implies that we obtain the simplified form :

$$
\begin{aligned}
& \int_{\Delta} \prod_{e=1}^{E} \frac{d \ell_{e}}{\ell_{e}}\left(\int_{\left(\mathbb{R}^{d}\right)^{n}} \prod_{e=1}^{E} \exp \left(-\frac{\mathbf{d}^{2}\left(x_{i(e)}, x_{j(e)}\right)}{4 \ell_{e}}\right) \psi\left(\mathbf{d}^{2}\left(x_{i(e)}, x_{j(e)}\right)\right) \ell_{e}^{s_{e}+k_{e}-\frac{d}{2}} a_{k_{e}}\left(x_{i(e)}, x_{j(e)}\right) \tilde{\varphi} d^{d} x_{1} \ldots d^{d} x_{n}\right) \\
= & \int_{[0,1]^{E}} \prod_{e=1}^{E} \frac{d t_{e}}{t_{e}} t_{e}^{\left(\sum_{i \leqslant e} 2 s_{i}+2 k_{i}\right)-d b_{1}\left(G_{e}\right)} \int_{\left(\mathbb{R}^{d}\right)^{n}} A\left(t_{e}, x, h_{e}\right) d^{d} x \prod_{e \in E(T)} d^{d} h_{e}
\end{aligned}
$$

where

$$
A\left(\left(t_{e}\right)_{e=1}^{E}, x,\left(h_{e}\right)_{e \in E(T)}\right)=2^{E} \pi^{*}\left(\left(\prod_{e=1}^{E} \exp \left(-\frac{\mathbf{d}^{2}\left(x_{i(e)}, x_{j(e)}\right)}{4 \ell_{e}}\right) \psi\left(\mathbf{d}^{2}\left(x_{i(e)}, x_{j(e)}\right)\right) a_{k_{e}}\right) \tilde{\varphi}\right) .
$$

The coordinates $\left(\left(t_{e}\right)_{e=1}^{E}, x,\left(h_{e}\right)_{e \in E(T)}\right)$ on $[0,1]^{E} \times \mathbb{R}^{d} \times\left(\mathbb{R}^{d}\right)^{E(T)}$ will be shortly denoted by $(t, x, h)$ for simplicity. We now prove the smoothness in $t \in[0,1]^{E}$ of the partial integral $\int_{\left(\mathbb{R}^{d}\right)^{n}} A(t, x, h) d^{d} x \prod_{e \in E(T)} d^{d} h_{e}$ which is needed to ensure analytic continuation.

Lemma 5.3 The map $t \mapsto \int_{\left(\mathbb{R}^{d}\right)^{n}} A(t, x, h) d^{d} x \prod_{e \in E(T)} d^{d} h_{e}$ belongs to $C^{\infty}\left([0,1]^{E}\right)$.

Proof - The smoothness of $A$ is a direct consequence of Theorem 5.2 . Now start from the definition of $A$ :

$$
\begin{aligned}
A(t, x, h) & =2^{E} \pi^{*}\left(\prod_{e \in E(T)} \exp \left(-\frac{\mathbf{d}^{2}\left(x_{i(e)}, x_{j(e)}\right)}{4 \ell_{e}}\right) \psi\left(\mathbf{d}^{2}\left(x_{i(e)}, x_{j(e)}\right)\right) a_{k_{e}}\right) \\
& \times \pi^{*}\left(\prod_{e \notin E(T)} \exp \left(-\frac{\mathbf{d}^{2}\left(x_{i(e)}, x_{j(e)}\right)}{4 \ell_{e}}\right) \psi\left(\mathbf{d}^{2}\left(x_{i(e)}, x_{j(e)}\right)\right) a_{k_{e}}\right) \\
& \times \tilde{\varphi}\left(x+\sum_{e \in(r, 1)}\left(\prod_{j \geqslant e} t_{j}\right) h_{e}, \ldots, x+\sum_{e \in(r, n)}\left(\prod_{j \geqslant e} t_{j}\right) h_{e}\right) .
\end{aligned}
$$

Then use the key fact that since the open neighborhood $\left(\cong \mathbb{R}^{d}\right)$ is chosen small enough and has compact closure, there exists a fixed constant $\delta>0$ such that for all $x, y \in \mathbb{R}^{d}$, we have the following bound on the Riemannian distance :

$$
\delta|x-y| \leqslant \mathbf{d}(x, y)=|x-y|+o(|x-y|) \leqslant \delta^{-1}|x-y|
$$

which follows from Lemma 5.2 since $\mathbf{d}^{2}(x, y)-\sum g_{\mu \nu}(x)\left(x^{\mu}-y^{\mu}\right)\left(x^{\nu}-y^{\nu}\right)$ vanishes along the diagonal at order 3 , and locally $\sum g_{\mu \nu}(x)\left(x^{\mu}-y^{\mu}\right)\left(x^{\nu}-y^{\nu}\right)$ is bounded by some multiple of $|x-y|$ by compactness of the neighborhood. It follows that

$$
\frac{\delta^{2}|x-y|^{2}}{4 \ell_{e}} \leqslant \frac{\mathbf{d}(x, y)^{2}}{4 \ell_{e}} \leqslant \frac{\delta^{-2}|x-y|^{2}}{4 \ell_{e}}
$$

which implies that for all edges $e \in E(T)$, we have the bound :

$$
\pi^{*} \exp \left(-\frac{\mathbf{d}^{2}\left(x_{i(e)}, x_{j(e)}\right)}{4 \ell_{e}}\right) \leqslant \pi^{*} \exp \left(-\frac{\delta^{2}|x-y|^{2}}{4 \ell_{e}}\right)=\exp \left(-\frac{\delta^{2}\left|h_{e}\right|^{2}}{4}\right) .
$$

This allows us to use the product $\pi^{*}\left(\prod_{e \in E(T)} \exp \left(-\frac{\mathbf{d}^{2}\left(x_{i(e)}, x_{j(e)}\right)}{4 \ell_{e}}\right)\right)$ to control the exponential decay of $A$ :

$$
\pi^{*}\left(\prod_{e \in E(T)} \exp \left(-\frac{\mathbf{d}^{2}\left(x_{i(e)}, x_{j(e)}\right)}{4 \ell_{e}}\right)\right) \leqslant \prod_{e \in E(T)} \exp \left(-\frac{\delta^{2}\left|h_{e}\right|^{2}}{4}\right) .
$$


By smoothness of the heat coefficients $a_{k}$, by compactness of the support of $\varphi \in C_{c}^{\infty}\left(U^{n}\right)$ thus of $\tilde{\varphi}$, and by the definition of $\pi$, for every multi-index $\alpha$, we find that there exists some constant $C_{\alpha}>0$ s.t.

$$
\left|\partial_{t}^{\alpha} \pi^{*}\left(\tilde{\varphi} \prod_{e \notin E(T)} \exp \left(-\frac{\mathbf{d}^{2}\left(x_{i(e)}, x_{j(e)}\right)}{4 \ell_{e}}\right) \prod_{e=1}^{E} a_{k_{e}} \psi\left(\mathbf{d}^{2}\left(x_{i(e)}, x_{j(e)}\right)\right)\right)\right| \leqslant C_{\alpha}\left(1+\sum_{e \in E(T)}\left|h_{e}\right|\right)^{|\alpha|},
$$

the partial derivatives in $t$ contributes the powers of $h$.

Therefore we have the bound for all $(t, x, h)$ :

$$
\left|\partial_{t}^{\alpha} A\left(t_{e}, x, h_{e}\right)\right| \leqslant C_{\alpha} \prod_{e \in E(T)} \exp \left(-\frac{\delta^{2}\left|h_{e}\right|^{2}}{4}\right)\left(1+\sum_{e \in E(T)}\left|h_{e}\right|\right)^{|\alpha|}
$$

with $A$ compactly supported in $x$. Then smoothness of $t \mapsto \int_{\left(\mathbb{R}^{d}\right)^{n}} A(t, x, h) d^{d} x \prod_{e \in E(T)} d^{d} h_{e}$ follows from smoothness of the integrand which is the function $A \in C^{\infty}\left([0,1]^{E} \times\left(\mathbb{R}^{d}\right)^{n}\right)$, and any derivative $\partial_{t}^{\alpha} A$ has fast decrease in $h$ when $|h| \rightarrow+\infty$ and compact support in the variable $x \in \mathbb{R}^{d}$. Therefore all derivatives in $\partial_{t}^{\alpha} A$ are integrable, uniformly in $t \in[0,1]^{E}$ and the conclusion follows from classical results on integrals depending smoothly on parameters.

Lemma 5.4 [Jet Lemma] Fix the sector $\Delta$ corresponding to the system of inequalities $\left\{0 \leqslant \ell_{1} \leqslant \cdots \leqslant\right.$ $\left.\ell_{E}\right\}$. Let

$$
A\left(\left(t_{e}\right)_{e=1}^{E}, x,\left(h_{e}\right)_{e \in T}\right)=\pi^{*}\left(\prod_{e=1}^{E}\left(\exp \left(-\frac{\mathbf{d}^{2}\left(x_{i(e)}, x_{j(e)}\right)}{4 \ell_{e}}\right) \psi\left(\mathbf{d}^{2}\left(x_{i(e)}, x_{j(e)}\right)\right) a_{k_{e}}\right) \tilde{\varphi}\right) .
$$

Then the $k$-jet of

$$
\chi\left(t_{1}, \ldots, t_{E}\right)=\int_{\left(\mathbb{R}^{d}\right)^{n}} A\left(\left(t_{e}\right)_{e=1}^{E}, x,\left(h_{e}\right)_{e \in E(T)}\right) d^{d} x \prod_{e \in E(T)} d^{d} h_{e}
$$

depends continuously on the $k$-jet of $\left(a_{k_{i}}, i=1, \cdots E, \varphi, \mathbf{d}^{2}, g\right)$.

Proof - The claim follows from the formulas defining $A$ and the change of variables $\pi$ and repeated application of the chain rule to $\pi^{*}\left(\prod_{e=1}^{E}\left(\exp \left(-\frac{\mathbf{d}^{2}\left(x_{i(e)}, x_{j(e)}\right)}{4 \ell_{e}}\right) \psi\left(\mathbf{d}^{2}\left(x_{i(e)}, x_{j(e)}\right)\right) a_{k_{e}}\right) \varphi\right)$.

Recall that from Theorem 4.1. the main Theorem 2.1 reduces to an analytic continuation result for the amplitudes $I_{G, \vec{k}}(s)$ corresponding to the labelled Feynman graphs $(G, \vec{k})$. The problem was that the integral formula for $I_{G, \vec{k}}(s)$ involved some product of heat kernels which required blow-ups which were performed in sectors. The following Proposition shows how the integral expression $I_{G, \vec{k}}(s)$ simplifies after blow-up :

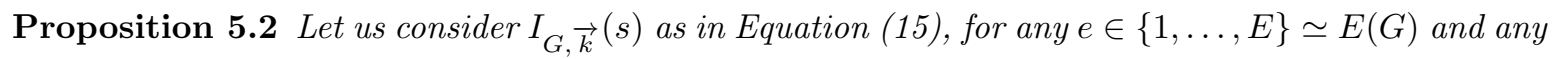
permutation $\sigma \in S_{E}, G_{\sigma(e)}$ is the subgraph of $G$ induced by the collection of edges $\{\sigma(1), \ldots, \sigma(e)\} \subset$ $E(G)$. Then for every test function $\varphi \in C_{c}^{\infty}\left(U^{n}\right)$, there exists a family $\chi_{\sigma} \in C^{\infty}\left([0,1]^{E}\right)$ indexed by $\sigma \in S_{E}$ such that the identity :

$$
\int_{\mathbb{R}^{d n}} I_{G, \vec{k}}(s) \varphi d^{n d} x=\prod_{e=1}^{E} \frac{1}{\Gamma\left(s_{e}\right)} \sum_{\sigma \in S_{E}} \int_{[0,1]} \prod_{e=1}^{E} \frac{d t_{e}}{t_{e}} t_{e}^{\sum_{i \leqslant e}\left(2 s_{\sigma(i)}+2 k_{\sigma(i)}\right)-d b_{1}\left(G_{\sigma(e)}\right)} \chi_{\sigma}(t)
$$

holds true for all $\operatorname{Re}\left(s_{e}\right), e \in\{1, \ldots, E\}$ large enough and both sides are holomorphic in $s \in \mathbb{C}^{E}$.

In the next paragraph, we will proceed to the meromorphic continuation of the r.h.s of equation (25) as meromorphic function with linear poles in $s$ and we will also bound the distributional order of $I_{G, \vec{k}}(s)$ independently of the label $\vec{k}$. 


\subsection{Integration by parts, bounding orders and pole decomposition.}

Now the proof of Theorem 2.1 on the analytic continuation of $t_{G}(s)$ is reduced to the meromorphic continuation in $s \in \mathbb{C}^{E}$ of the right hand side of Equation 25 . The meromorphic continuation comes from integration by parts as shown in the next Lemma and its corollary.

Lemma 5.5 Let $E$ be a positive integer, for any smooth function $\psi$ on $[0,1]$,

$$
I_{s}(\psi)=\int_{[0,1]^{E}} t_{1}^{s_{1}} \ldots t_{E}^{s_{E}} \psi\left(t_{1}, \ldots, t_{E}\right) d^{E} t
$$

can be analytically extended to a meromorphic germ at $\left(s_{e}=p_{e}\right)_{e=1}^{E} \in \mathbb{Z}^{E}$, more precisely, let $I=$ $\left\{i \mid p_{i}<0\right\}$

$$
\left(\prod_{i \in I}\left(s_{i}-p_{i}\right)\right) I_{s}(\psi)
$$

extends to a holomorphic germ at $\left(s_{e}=p_{e}\right)_{e}$ and $I_{s} \in \mathcal{D}^{\prime, m}\left([0,1]^{E}, \mathcal{M}_{s_{0}}\left(\mathbb{C}^{E}\right)\right), s_{0}=\left(p_{1}, \ldots, p_{E}\right)$, $m=\sum_{i \in I}\left|p_{i}\right|$.

The proof of this Lemma, given in the appendix, follows from integration by parts. One consequence of this Lemma is

Corollary 5.2 Denote by $1_{[0,1]^{E}}$ the indicator function of the unit cube $[0,1]^{E} \subset \mathbb{R}^{E}$. Let $\left(L_{1}, \cdots, L_{E}\right)$ be linear functions in $s \in \mathbb{C}^{E}$ with real coefficients $L_{i} \in\left(\mathbb{R}^{E}\right)^{*}, 1 \leqslant i \leqslant E$, and $\left(a_{1}, \ldots, a_{E}\right) \in \mathbb{Z}^{E}$. Set $I=\left\{i \mid a_{i}<0\right\}$, then

$$
\left(\prod_{i \in I} L_{i}(s)\right) \int_{[0,1]^{E}} t_{1}^{L_{1}(s)+a_{1}} \ldots t_{E}^{L_{E}(s)+a_{E}} \psi\left(t_{1}, \ldots, t_{E}\right) d^{E} t
$$

is a holomorphic germ at $s=0 \in \mathbb{C}^{E}$, and

$$
1_{[0,1]^{E}} t_{1}^{L_{1}(s)+a_{1}} \ldots t_{E}^{L_{E}(s)+a_{E}}
$$

extends to an element in $\mathcal{D}^{\prime, m}\left(\mathbb{R}^{E}, \mathcal{M}_{0}\left(\mathbb{C}^{E}\right)\right)$ where $m=\sum_{i \in I}\left|a_{i}\right|$ and the polar set is contained in $\left\{\prod_{i \in I} L_{i}=0\right\}$.

Applying Corollary 5.2 to the r.h.s. of Equation 25] shows that :

Lemma 5.6 Let $S_{\sigma}$ be the set of all subgraphs $H \in\left\{G_{\sigma(1)} \subset \cdots \subset G_{\sigma(E)}=G\right\}$ such that $b_{1}(H) \geqslant 1$, then

$$
\prod_{H \in S_{\sigma}}\left(\sum_{e \in E(H)} s_{e}-|E(H)|\right)\left(\int_{[0,1]} \prod_{e=1}^{E} \frac{d t_{e}}{t_{e}} t_{e}^{\sum_{i \leqslant e}\left(2 s_{\sigma(i)}+2 k_{\sigma(i)}\right)-d b_{1}\left(G_{\sigma(e)}\right)} \chi_{\sigma}(t)\right)
$$

is a holomorphic germ at $\left(s_{e}=1\right)_{e}$.

Proof - By Corollary 5.2 and a change of variables $s_{e}^{\prime}=s_{e}-1, e \in\{1, \cdots, E\}$, we need to consider the following set of indices :

$$
I=\left\{e \mid e+\sum_{i \leqslant e} k_{\sigma(i)}-\frac{d}{2} b_{1}\left(G_{\sigma(e)}\right) \leq 0\right\} \subset\{1, \ldots, E\}
$$

which is contained in $\left\{e \mid b_{1}\left(G_{\sigma(e)}\right) \geq 1\right\}$, which yields the conclusion. 
Let us comment on the above bound on the location of the pole. First the bound seems suboptimal since the set of indices $I=\left\{e \mid e+\sum_{i \leqslant e} k_{\sigma(i)}-\frac{d}{2} b_{1}\left(G_{\sigma(e)}\right) \leq 0\right\} \subset\{1, \ldots, E\}$ is only a subset of $\left\{e \mid b_{1}\left(G_{\sigma(e)}\right) \geq 1\right\} \subset\{1, \ldots, E\}$. However, it is important for us that we can give a bound on the location of the poles which does not depend on the multi-index $\vec{k}$ since poles from the original Feynman amplitude $t_{G}(s)$ do not depend on $\vec{k}$. The formula of Theorem 4.1 expresses $t_{G}$ as a sum of $I_{G, \vec{k}}$ for some $\vec{k}$. Hence the poles of $t_{G}(s)$ come from contributions from the poles of $I_{G, \vec{k}}$. Therefore it is convenient to have some $\vec{k}$ independent bound for poles of $I_{G, \vec{k}}$. Finally, we bound the distributional order of $I_{G, \vec{k}}$ and also give precise location on the affine planes supporting the poles of $I_{G, \vec{k}}$ in the following :

Proposition 5.3 (Poles of $I_{G, \vec{k}}$ and distributional order) Let $G$ be a graph whose set of edges is in bijection with $\{1, \ldots, E\}$. For any $e \in\{1, \ldots, E\} \simeq E(G)$ and any permutation $\sigma \in S_{E}$, $G_{\sigma(e)}$ is the subgraph of $G$ induced by the collection of edges $\{\sigma(1), \ldots, \sigma(e)\} \subset E(G)$. For every permutation $\sigma \in S_{E}$, we associate the filtration $\left\{G_{\sigma(1)} \subset \cdots \subset G_{\sigma(E)}=G\right\}$, and we consider the set $S_{\sigma}$ of all subgraphs $H \in\left\{G_{\sigma(1)} \subset \cdots \subset G_{\sigma(E)}=G\right\}$ such that $b_{1}(H) \geqslant 1$. For every $\vec{k} \in \mathbb{N}^{E(G)}$, the distribution $I_{G, \vec{k}}(s)$ defined in definition 15 can be analytically continued to $\mathcal{D}^{\prime, m}\left(U^{n}, \mathcal{M}_{s_{0}}\left(\mathbb{C}^{E}\right)\right)$, $s_{0}=\left(s_{e}=1\right)_{e=1}^{E}$ where

$$
m=\sum_{H \subset G ; 2|E(H)|-d b_{1}(H)-1<0} d b_{1}(H)-2|E(H)|+1 .
$$

For every test function $\varphi$,

$$
\int_{\mathbb{R}^{d n}} I_{G, \vec{k}}(s) \varphi d^{n d} x=\sum_{\sigma \in S_{E}} \prod_{H \in S_{\sigma}} \frac{1}{\left(\sum_{i \in H} s_{i}-E(H)\right)} f_{\sigma}(s)
$$

where $f_{\sigma}$ is holomorphic germ at $\left(s_{e}=1\right)_{e}$.

Remark 5.1 The bound on the distributional order depends only on the topology of the graph $G$ and the dimension $d$ and not on the element $\vec{k} \in \mathbb{N}^{E}$. The bound on the distributional order is also not sharp since we should only sum over subgraphs $G^{\prime} \in\left\{G_{\sigma(1)}, \ldots, G_{\sigma(E)}\right\}$ such that $2\left|E\left(G^{\prime}\right)\right|-d b_{1}\left(G^{\prime}\right)-1<0$ then take the supremum over all permutations $\sigma$.

Proof - We proved in Proposition 5.2 that for every labelled graph $(G, \vec{k})$, and every test function $\varphi \in C_{c}^{\infty}\left(U^{n}\right), n=|V(G)|$, there exists a family $\chi_{\sigma}(t)$ of smooth functions on the cube $[0,1]^{E}$ indexed by permutations $\sigma \in S_{E}$ such that:

$$
\int_{\mathbb{R}^{d n}} I_{G, \vec{k}}(s) \varphi d^{n d} x=\prod_{e=1}^{E} \frac{1}{\Gamma\left(s_{e}\right)} \sum_{\sigma \in S_{E}} \int_{[0,1]^{E}} \prod_{e=1}^{E} \frac{d t_{e}}{t_{e}} t_{e}^{\sum_{i \leqslant e}\left(2 s_{\sigma(i)}+2 k_{\sigma(i)}\right)-d b_{1}\left(G_{\sigma(e)}\right)} \chi_{\sigma}(t) .
$$

By applying Lemma 5.6 to

$$
\sum_{\sigma \in S_{E}} \int_{[0,1]^{E}} \prod_{e=1}^{E} \frac{d t_{e}}{t_{e}} t^{\sum_{i \leqslant e}\left(2 s_{\sigma(i)}+2 k_{\sigma(i)}\right)-d b_{1}\left(G_{\sigma(e)}\right)} \chi_{\sigma}(t)
$$

we obtain the meromorphic continuation of $s \mapsto \int_{\mathbb{R}^{d n}} I_{G, \vec{k}}(s) \varphi d^{n d} x$ with the bound on the location of poles. To show that $I_{G, \vec{k}}(s)$ is actually an element of $\mathcal{D}^{\prime, m}\left(U^{n}, \mathcal{M}_{s_{0}}\left(\mathbb{C}^{E}\right)\right), s_{0}=\left(s_{e}=1\right)_{e=1}^{E}$, we need to show that

$$
\varphi \in C_{c}^{\infty}\left(U^{n}\right) \mapsto \int_{\mathbb{R}^{d n}} I_{G, \vec{k}}(.) \varphi d^{n d} x \in \mathcal{M}_{s_{0}}\left(\mathbb{C}^{E}\right)
$$


depends linearly on the $m$-jet of $\varphi$ for some $m$. By Corollary 5.2 , the integral

$$
\int_{[0,1]^{E}} \prod_{e=1}^{E} \frac{d t_{e}}{t_{e}} t_{e}^{\sum_{i \leqslant e}\left(2 s_{\sigma(i)}+2 k_{\sigma(i)}\right)-d b_{1}\left(G_{\sigma(e)}\right)} \chi_{\sigma}(t)
$$

depends linearly on the $m$-jet of $\chi_{\sigma}$ for

$$
m=\sum_{G^{\prime} \subset G ; 2\left|E\left(G^{\prime}\right)\right|-d b_{1}\left(G^{\prime}\right)-1<0} d b_{1}\left(G^{\prime}\right)-2\left|E\left(G^{\prime}\right)\right|+1 .
$$

Then by Lemma 5.4 the $m$-jet of $\chi_{\sigma}$ depends continuously on the $m$-jet of $\varphi$ which yields the result.

Now let us restate the first main Theorem from our paper and conclude its proof :

Theorem 5.3 Let $(M, g)$ be a smooth, compact, connected Riemannian manifold without boundary of dimension $d, d v(x)$ the Riemannian volume and $P=-\Delta_{g}+V, V \in C_{\geqslant 0}^{\infty}(M)$ or $M=\mathbb{R}^{d}$ with a constant metric $g$ and $P=-\Delta_{g}+\lambda^{2}, \lambda \in \mathbb{R}_{\geqslant 0}$. Then for every graph $G$,

$$
t_{G}(s)=\prod_{e \in E(G)} \mathfrak{G}^{s_{e}}\left(x_{i(e)}, x_{j(e)}\right)
$$

can be analytically continued as an element of $\mathcal{D}^{\prime}\left(M^{V(G)}, \mathcal{M}_{s_{0}}\left(\mathbb{C}^{E(G)}\right)\right)$ where $s_{0}=\left(s_{e}=\right.$ $1)_{e \in E(G)}$, with linear poles supported on the union of affine hyperplanes

$$
\bigcup_{G^{\prime}}\left\{\sum_{e \in G^{\prime}} s_{e}-\left|E\left(G^{\prime}\right)\right|=0\right\}
$$

where the union runs over subgraphs $G^{\prime}$ of $G$ such that $2\left|E\left(G^{\prime}\right)\right|-b_{1}\left(G^{\prime}\right) d \leqslant 0$ and $\left|E\left(G^{\prime}\right)\right|$ is the number of edges in $G^{\prime}$.

Proof - From Theorem 4.1, one has a decomposition :

$$
\left.t_{G}(s)\right|_{U_{x}^{n}}=\sum_{G^{\prime} \subset G}(\sum_{\vec{k} \in\{0, \ldots, p\}^{E_{1}}} \underbrace{I_{G^{\prime}}, \vec{k}}(s)) \times h_{G \backslash G^{\prime}}(s)
$$

where the sum runs over subgraphs $G^{\prime} \subset G$ and $h_{G \backslash G^{\prime}}(s) \in C^{m}\left(M^{V(G)}, \mathcal{O}_{s_{0}}\left(\mathbb{C}^{E(G) \backslash E\left(G^{\prime}\right)}\right)\right), s_{0}=\left(s_{e}=\right.$ $1)_{e \in E(G) \backslash E\left(G^{\prime}\right)}$ as soon as $p>\frac{d+m}{2}-1$ for $m=\sum_{G^{\prime} \subset G ; 2\left|E\left(G^{\prime}\right)\right|-d b_{1}\left(G^{\prime}\right)-1<0} d b_{1}\left(G^{\prime}\right)-2\left|E\left(G^{\prime}\right)\right|+1$. Therefore, the analytic continuation of $t_{G}(s)$ should follow from the analytic continuation of $I_{G^{\prime}, \vec{k}}$ for all subgraphs $G^{\prime}$ of $G$ and the fact that the distributional order of $I_{G^{\prime}}, \vec{k}$ is bounded from above by some integer $m\left(G^{\prime}\right)$ independent of $\vec{k} \in \mathbb{N}^{E\left(G^{\prime}\right)}$. But Proposition 5.3 precisely gives us that the distributional order of every $I_{G^{\prime}}, \vec{k}$ is bounded from above by some integer which depends only on the topology of $G^{\prime}$. Now following the notations from Proposition 5.3 for every subgraph $G^{\prime} \subset G$, we denote by $S_{E\left(G^{\prime}\right)}$ the permutations of the edges $E\left(G^{\prime}\right)=\left\{1, \ldots, E^{\prime}\right\}$. For every permutation $\sigma \in S_{E\left(G^{\prime}\right)}$ corresponds a canonical filtration $\left\{G_{\sigma(1)}^{\prime} \subset \cdots \subset G_{\sigma\left(E^{\prime}\right)}^{\prime}\right\}$ of $G^{\prime}$ and $S_{\sigma}$ denotes the set of all subgraphs $H \in\left\{G_{\sigma(1)}^{\prime} \subset \cdots \subset G_{\sigma\left(E^{\prime}\right)}^{\prime}\right\}$ such that $b_{1}(H) \geqslant 1$. Finally doing all the bookkeeping, we find that

$$
t_{G}(s)=\sum_{G^{\prime} \subset G} \sum_{\sigma \in S_{E\left(G^{\prime}\right)}}\left(\prod_{H \in S_{\sigma}} \frac{1}{\left(\sum_{i \in H} s_{i}\right)-E(H)}\right) h_{\sigma}(s)
$$

where $h_{\sigma}(s) \in \mathcal{D}^{\prime}\left(M^{V(G)}, \mathcal{O}_{s_{0}}\left(\mathbb{C}^{E(G)}\right)\right), s_{0}=\left(s_{e}=1\right)_{e \in E(G)}$. 


\section{Renormalization of Feynman amplitudes}

In this second part of our paper, we shall apply the analytic continuation results derived in the previous part to the renormalization of Feynman amplitudes on Riemannian manifolds.

\subsection{Renormalization maps.}

For a smooth manifold $(M, g)$ and for every finite subset $I \subset \mathbb{N}$, we denote by $M^{I}$ the configuration space of points labelled by $I$. For $J \subset I$ with $|J| \geq 2, D_{J}$ is the subset $\left\{\left(x_{i}\right)_{i \in I}\right.$ s.t. $x_{j}=x_{k}$ for $j, k \in$ $J\}$ of $M^{I}$ called $J$-diagonal. Let $\Delta_{I}=\cup_{J \subset I,|J| \geq 2} D_{J}$ be the maximal diagonal.

Definition 6.1 (Labelling vertices) Let $I \subset \mathbb{N},|I|<+\infty$ be some finite subset of integers. A graph with vertices labelled by $I$ is a pair $(G, \iota)$, where $G$ is a graph, ८ is an injective map from $V(G)$ to $I$.

For a graph with vertices labelled by $I,(G, \iota)$, defines an element

$$
t_{G}=\prod_{e \in E(G)} \mathfrak{G}\left(x_{i(e)}, x_{j(e)}\right),
$$

where $(i(e), j(e)) \in I^{2}$ and $t_{G}$ is a smooth function on $M^{I} \backslash \Delta_{I}$. For a finite subset $I$ of $\mathbb{N}$, let us denote by $\mathcal{F}\left(M^{I}\right)$ the linear span of $t_{G}$ of all $(G, \iota)$ with $\iota(V(G)) \subset I$ as smooth functions on $M^{I} \backslash \Delta_{I}$.

For a linear map $\mathcal{R}: E \mapsto \mathcal{D}^{\prime}(M)$ where $E$ is a vector space and $M$ is a smooth manifold, and any open subset $U \subset M$, let $i_{U}: U \hookrightarrow M$ denote the inclusion map. Then $\left.\mathcal{R}\right|_{U}=i_{U}^{*} \mathcal{R}: E \mapsto \mathcal{D}^{\prime}(U)$ is the pull-back of $\mathcal{R}$ by $i_{U}$. Following recent work by Nikolov-Stora-Todorov [56], we can give a definition of renormalization as follows :

Definition 6.2 A renormalization is a sequence of (not necessarily continuous) linear maps $\mathcal{R}_{I}$ : $\mathcal{F}\left(M^{I}\right) \mapsto \mathcal{D}^{\prime}\left(M^{I}\right)$ indexed by finite subsets I of $\mathbb{N}$, which satisfies the following system of functional equations :

- For $I \subset J$, and $t \in \mathcal{F}\left(M^{I}\right)$,

$$
\mathcal{R}_{J}(t)=\mathcal{R}_{I}(t) .
$$

This is the compatibility condition for the family of linear maps.

- $\forall t \in \mathcal{F}\left(M^{I}\right), \varphi \in C_{c}^{\infty}\left(M^{I} \backslash \Delta_{I}\right)$,

$$
\left\langle\mathcal{R}_{I}(t), \varphi\right\rangle=\langle t, \varphi\rangle .
$$

This means that $\mathcal{R}_{I}(t)$ is a distributional extension of $t \in C^{\infty}\left(M^{I} \backslash \Delta_{I}\right)$.

- For a graph $(G, \iota)$ with vertices labelled by $J \subset \mathbb{N}$, and $I \subset J=\iota(V(G))$, set $I^{c}=J \backslash I$, let $E_{I}=\left\{e \in E(G) ; i(e), j(e) \in I^{2}\right\}, E_{I^{c}}=\left\{e \in E(G) ; i(e), j(e) \in I^{c 2}\right\}, E_{I I^{c}}=E(G) \backslash\left(E_{I} \cup E_{I^{c}}\right)$, and we denote by $G_{I}, G_{I^{c}}, G_{I^{c}}$ the corresponding induced subgraphs of $G$. For open subsets $U, V$ of $M$ with $\operatorname{dist}(U, V)>0$, denote by $U^{I} \times V^{I^{c}}$ the subset $\left\{\left(x_{j}\right)_{j \in J} \in M^{J}\right.$ s.t. $x_{i} \in U, \forall i \in I, x_{i} \in$ $\left.V, \forall i \in I^{c}\right\} \subset M^{J}$. Then :

$$
\left.\mathcal{R}_{J}\right|_{U^{I} \times V^{I^{c}}}\left(t_{G}\right)=\left(\left.\left.\mathcal{R}_{J}\right|_{U^{I}}\left(t_{G_{I}}\right) \otimes \mathcal{R}_{J}\right|_{V^{I^{c}}}\left(t_{G_{I^{c}}}\right)\right) t_{G_{I^{c}}}
$$

as distributions in $\mathcal{D}^{\prime}\left(U^{I} \times V^{I^{c}}\right)$. This means that renormalization must preserve locality.

- Let $\Phi: M \mapsto M$ be an orientation preserving diffeomorphism and we denote by $\Phi_{I}: M^{I} \mapsto M^{I}$ the induced diffeomorphism on configuration space $M^{I}$. We assume that the renormalization 
maps depend on the Riemannian metric $g$ and denote it by $\mathcal{R}[g]=\left(\mathcal{R}[g]_{I}\right)_{I}$ to stress this dependence. Then the covariance equation on renormalization maps reads for all graph $(G, \iota)$ with vertices labelled by $I$ :

$$
\mathcal{R}\left[\Phi^{*} g\right]_{I}\left(\Phi_{I}^{*} t_{G}\right)=\Phi_{I}^{*}\left(\mathcal{R}[g]_{I}\left(t_{G}\right)\right) .
$$

This axiom of functorial nature ensures the renormalization is covariant.

The following property follows from the locality condition : for a graph $(G, \iota)$ with vertices labelled by $I$, if $G$ is the disjoint union of $G_{1}$ and $G_{2}, \iota\left(V\left(G_{1}\right)\right) \subset I_{1}, \iota\left(V\left(G_{2}\right)\right) \subset I_{2}, I_{1} \cap I_{2}=\emptyset$ then :

$$
\mathcal{R}_{I_{1} \cup I_{2}}\left(t_{G}\right)=\mathcal{R}_{I_{1}}\left(t_{G_{1}}\right) \otimes \mathcal{R}_{I_{2}}\left(t_{G_{2}}\right)
$$

as distributions in $\mathcal{D}^{\prime}\left(M^{I_{1} \cup I_{2}}\right)$.

\subsection{Decompositions of meromorphic germs of distributions}

Our goal in this paragraph is to extend the decomposition of [38, (see also [13, Appendix]) of the space $\mathcal{M}_{s_{0}}$ of meromorphic germs with linear poles at $s_{0} \in \mathbb{R}^{p} \subset \mathbb{C}^{p}$ to their distributional counterpart $\mathcal{D}^{\prime}\left(., \mathcal{M}_{s_{0}}\right)$ defined in paragraph 3.2 This decomposition plays an essential role in our definition of renormalization maps by projections. Recall we denoted by $\mathcal{O}_{s_{0}}$ the space of holomorphic germs at $s_{0}$.

Let us fix a nondegenerate bilinear form

$$
Q(\cdot, \cdot): \mathbb{R}^{p} \times \mathbb{R}^{p} \rightarrow \mathbb{R}
$$

which induces a nondegenerate bilinear form

$$
Q^{*}(\cdot, \cdot):\left(\mathbb{R}^{p}\right)^{*} \times\left(\mathbb{R}^{p}\right)^{*} \rightarrow \mathbb{R} .
$$

We can now define the concept of polar germ [38, definition 2.3], a polar germ at $s_{0}$ is a meromorphic germ of the form $\frac{1}{L_{1}^{n_{1}}\left(s-s_{0}\right) \cdots L_{k}^{n_{k}}\left(s-s_{0}\right)} h\left(\ell\left(s-s_{0}\right)\right)$, where $L_{1}, \cdots, L_{k}$ are linearly independent linear functions in $\left(\mathbb{R}^{p}\right)^{*},\left(n_{1}, \cdots n_{k}\right) \in \mathbb{N}_{>0}^{k}, \ell=\left(\ell_{1}, \cdots, \ell_{n}\right): \mathbb{R}^{p} \rightarrow \mathbb{R}^{n}$ defined by linear functions $\ell_{1}, \cdots, \ell_{n}$, and $h$ is a holomorphic germ at $0 \in \mathbb{C}^{n}$, such that $Q^{*}\left(L_{i}, \ell_{j}\right)=0,1 \leqslant i \leqslant k, 1 \leqslant j \leqslant n$. Let $\mathcal{P}_{s_{0}}$ be the linear span of polar germs at $s_{0}$ in $\mathcal{M}_{s_{0}}$.

Notice the polar set is defined by real linear functions, by similar proof as in 38, using some geometry of cones, we have the following :

Proposition 6.1 There is a decomposition:

$$
\mathcal{M}_{s_{0}}=\mathcal{O}_{s_{0}} \oplus \mathcal{P}_{s_{0}}
$$

Now we extend the concept of polar germs to distributions valued in polar germs.

Definition 6.3 Let $M$ be a smooth manifold and $p \in \mathbb{N}$. A polar germ of distributions at $s_{0} \in \mathbb{R}^{p} \subset \mathbb{C}^{p}$ is some element of $\mathcal{D}^{\prime}\left(M, \mathcal{M}_{s_{0}}\left(\mathbb{C}^{p}\right)\right)$ of the form $\frac{1}{L_{1}^{n_{1}}\left(s-s_{0}\right) \cdots L_{k}^{n_{k}}\left(s-s_{0}\right)} h\left(\ell\left(s-s_{0}\right)\right)$, where $L_{1}, \cdots, L_{k}$ are linearly independent linear functions in $\left(\mathbb{R}^{p}\right)^{*},\left(n_{1}, \cdots n_{k}\right) \in \mathbb{N}_{>0}^{k}, \ell=\left(\ell_{1}, \cdots, \ell_{n}\right): \mathbb{R}^{p} \rightarrow \mathbb{R}^{n}$ defined by linear functions $\ell_{1}, \cdots, \ell_{n}$, and $h \in \mathcal{D}^{\prime}\left(M, \mathcal{O}_{0}\left(\mathbb{C}^{p}\right)\right)$ such that $Q^{*}\left(L_{i}, \ell_{j}\right)=0,1 \leqslant i \leqslant k, 1 \leqslant j \leqslant n$. We denote by $\mathcal{D}^{\prime}\left(M, \mathcal{P}_{s_{0}}\left(\mathbb{C}^{p}\right)\right)$ the linear span of polar germs of distributions at $s_{0}$ in $\mathcal{D}^{\prime}\left(M, \mathcal{M}_{s_{0}}\left(\mathbb{C}^{p}\right)\right)$.

Lemma 6.1 If $\frac{1}{L_{1}^{n_{1}}\left(s-s_{0}\right) \cdots L_{k}^{n_{k}}\left(s-s_{0}\right)} h\left(\ell\left(s-s_{0}\right)\right)$ and $\frac{1}{M_{1}^{m_{1}}\left(s-s_{0}\right) \cdots M_{p}^{m_{p}}\left(s-s_{0}\right)} g\left(\ell^{\prime}\left(s-s_{0}\right)\right)$ represent the same nonzero meromorphic germ of distributions, then $k=m p$, and $M_{1}, \cdots, M_{m}, L_{1}, \ldots, L_{k}$ can be rearranged in such a way that $L_{i}$ is a multiple of $M_{i}$ and $n_{i}=m_{i}$ for $1 \leq i \leq k$.

Proof - Since this meromorphic germ is not zero, we can take a test function $\varphi$ such that $h\left(\ell\left(s-s_{0}\right)\right)(\varphi)$ is not identically zero, then $\frac{1}{L_{1}^{n_{1}}\left(s-s_{0}\right) \cdots L_{k}^{n_{k}}\left(s-s_{0}\right)} h\left(\ell\left(s-s_{0}\right)\right)(\varphi)$ and $\frac{1}{M_{1}^{m_{1}}\left(s-s_{0}\right) \cdots M_{p}^{m_{p}}\left(s-s_{0}\right)} g\left(\ell^{\prime}(s-\right.$ $\left.s_{0}\right)(\varphi)$ represent the same polar germ, by the same proof as in [38, Lemma 2.8], we have the conclusion. 
We then prove the promised decomposition Theorem for $\mathcal{D}^{\prime}\left(M, \mathcal{M}_{s_{0}}\right)$ which generalizes the result in 38 .

Theorem 6.1 Let $M$ be some smooth manifold and $s_{0} \in \mathbb{R}^{p} \subset \mathbb{C}^{p}$. We have the direct sum decomposition :

$$
\mathcal{D}^{\prime}\left(M, \mathcal{M}_{s_{0}}\left(\mathbb{C}^{p}\right)\right)=\mathcal{D}^{\prime}\left(M, \mathcal{O}_{s_{0}}\left(\mathbb{C}^{p}\right)\right) \oplus \mathcal{D}^{\prime}\left(M, \mathcal{P}_{s_{0}}\left(\mathbb{C}^{p}\right)\right) .
$$

Proof - Without loss of generality, we can assume that $s_{0}=0$. For $t \in \mathcal{D}^{\prime}\left(M, \mathcal{M}_{0}\left(\mathbb{C}^{p}\right)\right)$, by definition, there exist $L_{1}, \cdots, L_{k} \in\left(\mathbb{R}^{p}\right)^{*}$ such that $L_{1} \cdots L_{k} t \in \mathcal{D}^{\prime}\left(M, \mathcal{O}_{0}\left(\mathbb{C}^{p}\right)\right)$. By partial fractions decompositions as in the proof of [38, Lemma 2.9 property a)], we may assume there is $\left(n_{1}, \ldots, n_{k}\right) \in \mathbb{N}_{>0}^{k}$ such that $L_{1}^{n_{1}} \cdots L_{k}^{n_{k}} t \in \mathcal{D}^{\prime}\left(M, \mathcal{O}_{0}\left(\mathbb{C}^{p}\right)\right)$ with $L_{1}, \cdots, L_{k}$ linearly independent and $\left(n_{1}, \cdots, n_{k}\right) \in \mathbb{N}_{>0}^{k}$.

Now let us expand $L_{1}, \cdots, L_{k}$ to a basis $\left(e_{1}, \ldots, e_{p}\right)$ of $\left(\mathbb{R}^{p}\right)^{*}$ with $e_{i}=L_{i}, 1 \leqslant i \leqslant k$ and $Q\left(e_{i}, e_{j}\right)=$ 0 for $1 \leqslant i \leqslant k, k+1 \leqslant j \leqslant p$. Then by Proposition 3.2 , we have the power series expansion for :

$$
z_{1}^{n_{1}} \cdots z_{k}^{n_{k}} t=\sum_{\alpha \in \mathbb{N}^{p}} \frac{z^{\alpha}}{\alpha !} t_{\alpha}
$$

where $z=\sum z_{i} e_{i}^{*} \in\left(\mathbb{C}^{p}\right)^{*}$. So when we apply $z_{1}^{n_{1}} \cdots z_{k}^{n_{k}} t$ against the test function $\varphi$, we obtain $z_{1}^{n_{1}} \cdots z_{k}^{n_{k}} t(\varphi)=\sum_{\alpha \in \mathbb{N}^{p}} \frac{z^{\alpha}}{\alpha !} t_{\alpha}(\varphi)$, which is absolutely convergent in a small neighborhood of $0 \in \mathbb{C}^{p}$.

Let $S=\left\{d=\left(d_{1}, \cdots, d_{p}\right) \in \mathbb{N}^{p} \mid d \neq(0, \ldots, 0), 0 \leqslant d_{i} \leqslant n_{i}\right\}$. For $d \in S$, let $I(d)=\left\{i \mid d_{i} \neq 0\right\} \subset$ $\{1, \ldots, p\}$, and set

$$
N_{d}=\left\{\alpha \in \mathbb{N}^{p} \mid \alpha_{i}=n_{i}-d_{i} \text { if } i \in I(d), \alpha_{i} \geq n_{i} \text { if } i \in\{1, \ldots, k\} \backslash I(d), \alpha_{i} \in \mathbb{N} \text { if } i \in\{k+1, \ldots, p\}\right\} .
$$

Then we note that $N_{d} \cap N_{e}=\emptyset$ if $d \neq e \in S$ and most importantly, we have the partition $\mathbb{N}^{p}=\bigcup_{d \in S} N_{d}$.

Now for $z_{i} \neq 0,1 \leqslant i \leqslant k$,

$$
t(\varphi)=\sum_{\alpha \in \mathbb{N}^{p}} \frac{z^{\alpha-\alpha_{0}}}{\alpha !} t_{\alpha}(\varphi)=\sum_{d \in S} \sum_{\alpha \in N_{d}} \frac{z^{\alpha-\alpha_{0}}}{\alpha !} t_{\alpha}(\varphi),
$$

where $\alpha_{0}=\left(n_{1}, \cdots, n_{k}, 0, \cdots, 0\right)$. And we have

$$
\sum_{\alpha \in N_{d}} \frac{z^{\alpha-\alpha_{0}}}{\alpha !} t_{\alpha}(\varphi)=\frac{1}{z_{I(d)}^{d}} \sum_{\alpha \in N_{d}} \frac{z_{[p] \backslash I(d)}^{\alpha-\alpha_{0}}}{\alpha !} t_{\alpha}(\varphi)
$$

where $z_{I(d)}^{d}=\prod_{i \in I(d)} z_{i}^{d_{i}}, z_{[p] \backslash I(d)}^{\alpha-\alpha_{0}}=\prod_{i \in\{1, \ldots, k\} \backslash I(d)} z_{i}^{\alpha_{i}-s_{i}} \prod_{i \in\{k+1, \ldots, p\}} z_{i}^{\alpha_{i}}$.

Let

$$
h_{d}=\sum_{\alpha \in N_{d}} \frac{z_{[p] \backslash I}^{\alpha-\alpha_{0}}}{\alpha !} t_{\alpha}
$$

By Proposition 3.2, for every compact $K \subset U$ there exists $C>0$ and some continuous seminorm $P$ for the Fréchet topology of $C_{K}^{\infty}(U)$ such that : $\forall \varphi \in C_{K}^{\infty}(U),\left|t_{\alpha}(\varphi)\right| \leqslant \frac{\alpha !}{r^{|\alpha|}} C P(\varphi)$ for all multi-indices $\alpha \in \mathbb{N}^{p}$. This implies that for every $0<R<r$, for all $|z|<R$,

$$
\left|\sum_{\alpha \in \mathbb{N}^{p}} \frac{z^{\alpha}}{\alpha !} t_{\alpha}(\varphi)\right| \leqslant \sum_{\alpha \in \mathbb{N}^{p}}\left|\frac{z^{\alpha}}{\alpha !} t_{\alpha}(\varphi)\right| \leqslant \sum_{\alpha \in \mathbb{N}^{p}} \frac{R^{|\alpha|}}{\alpha !} \frac{\alpha !}{r|\alpha|} C P(\varphi)=\left(1-\frac{R}{r}\right)^{-p} C P(\varphi) .
$$

Therefore, $\left|\left(\prod_{i \in[k] \backslash I(d)} z_{i}^{s_{i}}\right) h_{d}(\varphi)\right| \leqslant \sum_{\alpha \in \mathbb{N}^{p}}\left|\frac{z^{\alpha}}{\alpha !} t_{\alpha}(\varphi)\right| \leqslant\left(1-\frac{R}{r}\right)^{-p} C P(\varphi)$, so $\left(\prod_{i \in[k] \backslash I(d)} z_{i}^{s_{i}}\right) h_{d} \in$ $\mathcal{D}^{\prime}\left(M, \mathcal{O}_{0}\left(\mathbb{C}^{p}\right)\right)$, so $h_{d} \in \mathcal{D}^{\prime}\left(M, \mathcal{O}_{0}\left(\mathbb{C}^{p}\right)\right)$.

Now by definition, $\frac{1}{z_{I(d)}^{d}} h_{d}$ is a polar germ of distributions if $d \neq(0, \cdots, 0), h_{(0, \cdots, 0)} \in \mathcal{D}^{\prime}\left(M, \mathcal{O}_{0}\left(\mathbb{C}^{p}\right)\right)$, and

$$
t=\sum_{d \in S} \frac{1}{z_{I(d)}^{d}} h_{d} \in \mathcal{D}^{\prime}\left(M, \mathcal{P}_{0}\left(\mathbb{C}^{p}\right)\right)+\mathcal{D}^{\prime}\left(M, \mathcal{O}_{0}\left(\mathbb{C}^{p}\right)\right)
$$


where the singular part reads as a finite sum of polar germs as a corollary of the above argument. So we have $\mathcal{D}^{\prime}\left(M, \mathcal{M}_{s_{0}}\left(\mathbb{C}^{p}\right)\right)=\mathcal{D}^{\prime}\left(M, \mathcal{O}_{s_{0}}\left(\mathbb{C}^{p}\right)\right)+\mathcal{D}^{\prime}\left(M, \mathcal{P}_{s_{0}}\left(\mathbb{C}^{p}\right)\right)$. To show it is a direct sum, if $t \in \mathcal{D}^{\prime}\left(M, \mathcal{O}_{s_{0}}\left(\mathbb{C}^{p}\right)\right) \cap \mathcal{D}^{\prime}\left(M, \mathcal{P}_{s_{0}}\left(\mathbb{C}^{p}\right)\right)$, then for any test function $\phi, t(\phi) \in \mathcal{P}_{s_{0}} \cap \mathcal{O}_{s_{0}}$, so $t(\phi)=0$ by Proposition 6.1, which implies $t=0$.

A consequence of the decomposition Theorem is the following

Proposition 6.2 Let $M$ be a smooth manifold, $p \in \mathbb{N}$ and $s_{0} \in \mathbb{R}^{p} \subset \mathbb{C}^{p}$. There exists a projection

$$
\pi_{p}: \mathcal{D}^{\prime}\left(M, \mathcal{M}_{s_{0}}\left(\mathbb{C}^{p}\right)\right) \mapsto \mathcal{D}^{\prime}\left(M, \mathcal{O}_{s_{0}}\left(\mathbb{C}^{p}\right)\right)
$$

which sends distribution valued in meromorphic germs at $s_{0}$ to distribution valued in holomorphic germs at $s_{0}$ such that $\operatorname{ker}\left(\pi_{p}\right)=\mathcal{D}^{\prime}\left(M, \mathcal{P}_{s_{0}}\left(\mathbb{C}^{p}\right)\right)$.

Remark 6.1 Note that $\pi_{p}$ is uniquely determined by the vector subspace of polar germs which are in turn uniquely determined by the choice of the canonical quadratic form $Q: \mathbb{R}^{p} \times \mathbb{R}^{p} \rightarrow \mathbb{R}$ that we fixed at the beginning of the present section.

In appendix 7.2. we show some useful Lemmas on the functorial properties of the projection $\pi_{p}$ for $p \in \mathbb{N}$. As a consequence of $\left[38\right.$, we have a similar projection, still denoted a bit abusively by $\pi_{p}$, at the germ level : $\pi_{p}: \mathcal{M}_{s_{0}} \mapsto \mathcal{O}_{s_{0}}$. It follows that the two projectors are related by the following equation :

Corollary 6.1 Let $X$ be a smooth manifold and $s_{0} \in \mathbb{R}^{p} \subset \mathbb{C}^{p}$. For all $t(s) \in \mathcal{D}^{\prime}\left(X, \mathcal{M}_{s_{0}}\left(\mathbb{C}^{p}\right)\right)$ and for all test function $\varphi \in C_{c}^{\infty}(X)$,

$$
\left(\pi_{p} t(s)\right)(\varphi)=\pi_{p}(t(s)(\varphi))
$$

\subsection{A renormalization map by projections}

From now on, for any integer $p$, we fix the canonical quadratic form $Q$ on $\mathbb{R}^{p}: Q(x)=\sum_{i=1}^{p}\left|x_{i}\right|^{2}$ and we study germs at $s_{0}=(1, \ldots, 1) \in \mathbb{R}^{p}$. We denote by $\left.\mathbf{e v}\right|_{s_{0}}$ the evaluation of some holomorphic germ at $s_{0}$. The properties of the family of projections $\left(\pi_{p}\right)_{p \in \mathbb{N}}$ allow to us to give a definition of renormalization maps as follows :

Definition 6.4 (Renormalization maps by projections) For $I \subset \mathbb{N}$, we define the renormalization map $\mathcal{R}_{I}$ as follows: for a graph $(G, \iota)$ with vertices labelled by $I$,

$$
\mathcal{R}_{I}\left(t_{G}\right)=\left.\mathbf{e v}\right|_{s_{0}}\left(\pi_{|E(G)|}\left(t_{G}(s)\right)\right)
$$

where $\mathfrak{G}^{s}$ is the Schwartz kernel of $(-\Delta)^{-s}$.

Theorem 6.2 (Renormalization Theorem) Let $(M, g)$ be a smooth, compact, connected Riemannian manifold without boundary of dimension $d, d v(x)$ the Riemannian volume and $P=-\Delta_{g}+V$, $V \in C_{\geqslant 0}^{\infty}(M)$ or $M=\mathbb{R}^{d}$ with a constant metric $g$ and $P=-\Delta_{g}+m^{2}, m \in \mathbb{R}_{\geqslant 0}$. For every finite subset $I \subset \mathbb{N}$, for every graph $(G, \iota)$ with vertices labelled by $I \subset \mathbb{N}$, we define $\mathcal{R}_{I}\left(t_{G}\right) \in \mathcal{D}^{\prime}\left(M^{I}\right)$ as in definition 6.4 and extend it by linearity to the vector space $\mathcal{F}\left(M^{I}\right)$.

Then the collection of renormalization maps $\left(\mathcal{R}_{I}\right)_{I \subset \mathbb{N},|I|<+\infty}$ satisfies the functional equations of definition 6.2.

Proof - The compatibility condition is encoded in the family of projections. For simplicity of notations, we choose to drop the subindex $s_{0}$ for the space of germs so we will write $\mathcal{M}, \mathcal{O}$ instead of $\mathcal{M}_{s_{0}}, \mathcal{O}_{s_{0}}$ and it will always be understood from the context that we consider holomorphic and meromorphic germs localized at $s_{0}=(1, \ldots, 1) \in \mathbb{C}^{p}$ for some $p \in \mathbb{N}$. Furthermore, we also write $\pi$ for 
the projections instead of $\pi_{|E(G)|}$ where it will be understood that for every graph $G, \pi\left(t_{G}(s)\right)$ means $\pi_{|E(G)|}\left(t_{G}(s)\right)$.

We now prove that $\mathcal{R}_{I}\left(t_{G}\right)$ is a distributional extension of $t_{G}$. By Lemma 4.1, on $M^{I} \backslash \Delta_{I}$, for every $e \in E(G)$, every Green function $\mathfrak{G}^{s_{e}} \in C^{\infty}\left(M^{I} \backslash \Delta_{I}, \mathcal{O}\right)$ are in fact smooth and depend holomorphically on $s_{e}$. We also have the convergence $\mathfrak{G}^{s_{e}} \rightarrow \mathfrak{G}$ in $C^{\infty}\left(M^{2} \backslash \Delta_{2}\right)$ when $s_{e} \rightarrow 1$. Therefore, for any test function $\varphi \in C_{c}^{\infty}\left(M^{I} \backslash \Delta_{I}\right)$, by Corollary 6.1. $\pi\left(t_{G}(s)\right)(\varphi)=\pi\left(t_{G}(s)(\varphi)\right)=t_{G}(s)(\varphi)$ since $t_{G}(s)(\varphi)$ is holomorphic at $s_{0}=\left(s_{e}=1\right)_{e \in E(G)} \in \mathbb{C}^{E(G)}$, and

$$
\mathcal{R}_{I}\left(t_{G}\right)(\varphi)=\left.\mathbf{e v}\right|_{s_{0}} \pi\left(t_{G}(s)\right)(\varphi)=\left.\mathbf{e v}\right|_{s_{0}} t_{G}(s)(\varphi)=t_{G}(\varphi) .
$$

Now let us prove the locality. For a graph $(G, \iota)$ with vertices labelled by $J \subset \mathbb{N}$, and $I \subset$ $J=\iota(V(G))$, set $I^{c}=J \backslash I$, let $E_{I}=\left\{e \in E(G) ; i(e), j(e) \in I^{2}\right\}, E_{I^{c}}=\{e \in E(G) ; i(e), j(e) \in$ $\left.I^{c 2}\right\}, E_{I I^{c}}=E(G) \backslash\left(E_{I} \cup E_{I^{c}}\right)$, and we denote by $\left(G_{I}, G_{I^{c}}, G_{I I^{c}}\right)$ the corresponding induced subgraphs of $G$. Start from $t_{G}(s)=t_{G_{I}}\left(s_{I}\right) t_{G_{I^{c}}}\left(s_{I^{c}}\right) t_{G_{I I^{c}}}\left(s_{I^{c}}\right)$ where $s=\left(s_{e}\right)_{e \in E(G)}, s_{I}=\left(s_{e}\right)_{e \in E_{I}}, s_{I^{c}}=$ $\left(s_{e}\right)_{e \in E_{I^{c}}}, s_{I I^{c}}=\left(s_{e}\right)_{e \in E_{I I^{c}}}$. For a pair of disjoint open subsets $(U, V)$ such that $\operatorname{dist}(U, V)>0$, denote by $\left\{\left(x_{j}\right)_{j \in J} \in M^{J}\right.$ s.t. $\left.x_{i} \in U, \forall i \in I, x_{i} \in V, \forall i \in I^{c}\right\}$ the open subset of configuration space $M^{J}$. Then note that the product $t_{G_{I I^{c}}}\left(s_{I I^{c}}\right)=\prod_{e \in E_{I I^{c}}} \mathfrak{G}^{s_{e}}\left(x_{i(e)}, x_{j(e)}\right) \in C^{\infty}\left(U^{I} \times V^{I^{c}}, \mathcal{O}\left(\mathbb{C}^{E_{I I^{c}}}\right)\right)$. It follows by Lemma 7.3 proved in the appendix that

$$
\pi\left(t_{G}(s)\right)=\pi\left(t_{G_{I}}\left(s_{I}\right) t_{G_{I^{c}}}\left(s_{I^{c}}\right) t_{G_{I I^{c}}}\left(s_{I I^{c}}\right)\right)=\pi\left(t_{G_{I}}\left(s_{I}\right) t_{G_{I^{c}}}\left(s_{I^{c}}\right)\right) t_{G_{I^{c}}}\left(s_{I I^{c}}\right) .
$$

Now the distributions $t_{G_{I}}\left(s_{I}\right) \in \mathcal{D}^{\prime}\left(U^{I}, \mathcal{M}\left(\mathbb{C}^{E_{I}}\right)\right)$ and $t_{G_{I^{c}}}\left(s_{I^{c}}\right) \in \mathcal{D}^{\prime}\left(V^{I^{c}}, \mathcal{M}\left(\mathbb{C}^{E_{I^{c}}}\right)\right)$ depend on different variables, therefore by Lemma $7.2 \pi\left(t_{G_{I}}\left(s_{I}\right) \otimes t_{G_{I^{c}}}\left(s_{I^{c}}\right)\right)=\pi\left(t_{G_{I}}\left(s_{I}\right)\right) \otimes \pi\left(t_{G_{I^{c}}}\left(s_{I^{c}}\right)\right)$. Then as distributions on $U^{I} \times V^{I^{c}}$ :

$$
\begin{aligned}
\mathcal{R}_{J}\left(t_{G}\right) & =\left.\mathbf{e v}\right|_{\left(s_{e}=1\right)_{e \in E(G)}}\left(\pi\left(t_{G_{I}}\left(s_{I}\right)\right) \otimes \pi\left(t_{G_{I^{c}}}\left(s_{I^{c}}\right)\right) \times t_{G_{I I^{c}}}\left(s_{I I^{c}}\right)\right) \\
& =\left.\left.\left.\mathbf{e v}\right|_{\left(s_{e}=1\right)_{e \in E_{I}}} \pi\left(t_{G_{I}}\left(s_{I}\right)\right) \mathbf{e v}\right|_{\left(s_{e}=1\right)_{e \in E_{I}}} \pi\left(t_{G_{I^{c}}}\left(s_{I^{c}}\right)\right) \mathbf{e v}\right|_{\left(s_{e}=1\right)_{e \in E_{I I}}} t_{G_{I I^{c}}}\left(s_{I I^{c}}\right) \\
& =\left.\mathcal{R}_{I}\left(t_{G_{I}}\right) \mathcal{R}_{I^{c}}\left(t_{G_{I^{c}}}\right) t_{G_{I^{c}}}\right|_{U^{I} \times V^{I^{c}}}
\end{aligned}
$$

where $t_{G_{I^{c}}}$ is smooth on $U^{I} \times V^{I^{c}}$ which yields the desired equation.

\section{Appendix: technical details.}

\subsection{Proof of Proposition 3.2 .}

Proof - Without loss of generality assume that $z=0$. By definition and the multidimensional Cauchy's formula [37, p. 3], for any polydisc $D_{1} \times \cdots \times D_{p}$ around $z=0$, for any test function $\varphi \in C_{c}^{\infty}(M)$ and any $\lambda$ in the polydisc :

$t(\lambda)(\varphi)=\frac{1}{(2 \pi i)^{p}} \int_{\partial D_{1}} \ldots \int_{\partial D_{p}} \frac{t(z)(\varphi) d z_{1} \ldots d z_{p}}{\left(z_{1}-\lambda_{1}\right) \ldots\left(z_{p}-\lambda_{p}\right)}=\frac{1}{(2 \pi i)^{p}} \int_{\partial D_{1}} \ldots \int_{\partial D_{p}} \sum_{\alpha} \lambda^{\alpha} \frac{t(z)(\varphi) d z_{1} \ldots d z_{p}}{z_{1}^{\alpha_{1}+1} \ldots z_{p}^{\alpha_{p}+1}}$.

So for any multi-index $\alpha$ and any test function $\varphi$, we define the functional $t_{\alpha}$ by

$$
t_{\alpha}(\varphi)=\frac{\alpha !}{(2 \pi i)^{p}} \int_{\partial D_{1}} \ldots \int_{\partial D_{p}} \frac{t(z)(\varphi) d z_{1} \ldots d z_{p}}{z_{1}^{\alpha_{1}+1} \ldots z_{p}^{\alpha_{p}+1}}
$$

then the series $\sum_{|\alpha| \geqslant 0} \frac{s^{\alpha}}{\alpha !} t_{\alpha}(\varphi)$ converges absolutely to $t(s)(\varphi)$.

First note that the functional $t_{\alpha}$ is linear, it remains to prove that $t_{\alpha}$ is continuous. For that, it suffices to show that for every compact $K \subset M$, the restriction of $t_{\alpha}$ to the Fréchet space $C_{K}^{\infty}(M)$ of test functions supported in $K$ is continuous. For fixed $s, t(s)$ is linear continuous on $C_{K}^{\infty}(M)$ therefore there exists some constant $C(s)$ and continuous seminorm $P$ of $C_{K}^{\infty}(M)$ such that $|t(s)(\varphi)| \leqslant C(s) P(\varphi), \forall \varphi \in$ 
$C_{K}^{\infty}(M)$. Conversely for fixed $\varphi, s \in D_{1} \times \cdots \times D_{p} \mapsto t(s)(\varphi)$ is bounded by holomorphicity. By an application of the uniform boundedness principle since $C_{K}^{\infty}(M)$ is Fréchet, for every compact $K \subset M$, there exists $C>0$ and some continuous seminorm $P$ for the Fréchet topology of $C_{K}^{\infty}(M)$ such that:

$$
\forall \varphi \in C_{K}^{\infty}(M), \sup _{s \in \partial D_{1} \times \cdots \times \partial D_{p}}|t(s)(\varphi)| \leqslant C P(\varphi) .
$$

Assuming that all discs $\partial D_{i}$ have radius $r$, it immediately follows that $t_{\alpha}$ satisfies a distributional version of Cauchy's bound:

$$
\forall \varphi \in C_{K}^{\infty}(M),\left|t_{\alpha}(\varphi)\right| \leqslant \frac{\alpha !}{r^{|\alpha|}} C P(\varphi) .
$$

This also implies that for all $\varphi \in C_{K}^{\infty}(M)$, the power series $\sum_{\alpha} \frac{s^{\alpha}}{\alpha !} t_{\alpha}(\varphi)$ converges for $|\lambda|<r$ i.e. the convergence radius equals $r$.

\subsection{Products of meromorphic germ of distributions in different variables.}

In this part, we prove some useful Lemmas on products of meromorphic germ of distributions in different variables.

Lemma 7.1 Let $\left(X_{1}, X_{2}\right)$ be smooth manifolds, $\mu_{1} \in \mathbb{R}^{p_{1}} \subset \mathbb{C}^{p_{1}}$ and $\mu_{2} \in \mathbb{R}^{p_{2}} \subset \mathbb{C}^{p_{2}}$. If $t_{1}\left(s_{1}\right) \in$ $\mathcal{D}^{\prime}\left(X_{1}, \mathcal{M}_{\mu_{1}}\left(\mathbb{C}^{p_{1}}\right)\right)$ and $t\left(s_{2}\right) \in \mathcal{D}^{\prime}\left(X_{2}, \mathcal{M}_{\mu_{2}}\left(\mathbb{C}^{p_{2}}\right)\right)$ then the external tensor product $t_{1}\left(s_{1}\right) \otimes t_{2}\left(s_{2}\right)$ is a well-defined element in $\mathcal{D}^{\prime}\left(X_{1} \times X_{2}, \mathcal{M}_{\left(\mu_{1}, \mu_{2}\right)}\left(\mathbb{C}^{p_{1}+p_{2}}\right)\right)$.

Proof - Denote by $d v_{1}, d v_{2}$ some smooth densities on $X_{1}, X_{2}$ respectively. Since every compact subset $K \subset X_{1} \times X_{2}$, can be covered by some finite number of products of compacts of the form $K_{1} \times K_{2}$, by Lemma 4.2 it suffices to show that for all compacts $K_{1} \subset X_{1}, K_{2} \subset X_{2}$, the element $\left.t\left(s_{1} ; x\right) t\left(s_{2} ; y\right)\right|_{K_{1} \times K_{2}}$ is a well-defined meromorphic family of distribution in $\mathcal{D}^{\prime}\left(K_{1} \times K_{2}\right)$ at $\left(\mu_{1}, \mu_{2}\right) \in \mathbb{C}^{p_{1}} \times \mathbb{C}^{p_{2}}$ with linear poles. Hence we can assume, without loss of generality, that we work over some product $K_{1} \times K_{2} \subset X_{1} \times X_{2}$ of compact subsets and we assume without loss of generality that we work around $\left(\mu_{1}, \mu_{2}\right)=(0,0)$. There exists mononomials $P\left(s_{1}\right)=L_{1}\left(s_{1}\right) \ldots L_{k}\left(s_{1}\right)$ and $Q\left(s_{2}\right)=M_{1}\left(s_{2}\right) \ldots M_{l}\left(s_{2}\right)$, where $\left(L_{i}\right)_{i=1}^{k},\left(M_{i}\right)_{i=1}^{l}$ are linear functions, such that $P\left(s_{1}\right) t_{1}\left(s_{1}\right)$ and $Q\left(s_{2}\right) t_{2}\left(s_{2}\right)$ are holomorphic germs of distributions at $s_{1}=\mu_{1}, s_{2}=\mu_{2}$ respectively. Therefore by Proposition 3.2, we know that $P\left(s_{1}\right) t_{1}\left(s_{1}\right)$ and $Q\left(s_{2}\right) t_{2}\left(s_{2}\right)$ both admit Laurent series expansions $P\left(s_{1}\right) t_{1}\left(s_{1}\right)=\sum_{\alpha_{1}} s_{1}^{\alpha_{1}} u_{\alpha_{1}}, Q\left(s_{2}\right) t_{2}\left(s_{2}\right)=\sum_{\alpha_{2}} s_{2}^{\alpha_{2}} v_{\alpha_{2}}$ where there exists two integers $\left(m_{1}, m_{2}\right)$ corresponding to the distributional orders of $\left(\left.t_{1}\right|_{K_{1}},\left.t_{2}\right|_{K_{2}}\right)$ and two positive real numbers $\left(r_{1}, r_{2}\right)$ such that for all multi-index $\left(\alpha_{1}, \alpha_{2}\right) \in \mathbb{N}^{p_{1}+p_{2}}$, we have the bounds :

$$
\left\|u_{\alpha_{1}}\right\|_{\left(C^{m_{1}}\right)^{\prime}} \leqslant C_{1} r_{1}^{\left|\alpha_{1}\right|}, \quad\left\|v_{\alpha_{2}}\right\|_{\left(C^{\left.m_{2}\right)^{\prime}}\right.} \leqslant C_{2} r_{2}^{\left|\alpha_{2}\right|} .
$$

We define the series $P\left(s_{1}\right) t_{1}\left(s_{1}\right) \otimes Q\left(s_{2}\right) t_{2}\left(s_{2}\right)=\sum_{\alpha_{1}, \alpha_{2}} s_{1}^{\alpha_{1}} s_{2}^{\alpha_{2}} u_{\alpha_{1}} \otimes v_{\alpha_{2}}$ and we shall prove that the above series converges for $\left|s_{1}\right|+\left|s_{2}\right|$ small enough in the sense that for every test function $\varphi\left(x_{1}, x_{2}\right)$ supported in $K_{1} \times K_{2}$, the series

$$
\sum_{\alpha_{1}, \alpha_{2}} s_{1}^{\alpha_{1}} s_{2}^{\alpha_{2}} u_{\alpha_{1}} \otimes v_{\alpha_{2}}(\varphi)=\sum_{\alpha_{1}, \alpha_{2}} s_{1}^{\alpha_{1}} s_{2}^{\alpha_{2}} \int_{X_{1} \times X_{2}} u_{\alpha_{1}}\left(x_{1}\right) v_{\alpha_{2}}\left(x_{2}\right) \varphi\left(x_{1}, x_{2}\right) d v_{1}\left(x_{1}\right) d v_{2}\left(x_{2}\right)
$$

converges absolutely. We first prove it for some element $\varphi=\varphi_{1} \otimes \varphi_{2} \in C_{K_{1}}^{\infty}\left(X_{1}\right) \otimes C_{K_{2}}^{\infty}\left(X_{2}\right) \subset$ $C_{K_{1} \times K_{2}}^{\infty}\left(X_{1} \times X_{2}\right)$ which is a tensor product of two elements. For $s \in \mathbb{C}^{p}$, we shall use the notation $\|s\|=\sup _{j \in\{1, \ldots, p\}}\left|s_{j}\right|$ and for some multi-index $\alpha \in \mathbb{N}^{p},|\alpha|=\sum_{j=1}^{p} \alpha_{j}$. Then the series converges 
thanks to the bound :

$$
\begin{aligned}
& \left|\sum_{\alpha_{1}, \alpha_{2}} s_{1}^{\alpha_{1}} s_{2}^{\alpha_{2}} \int_{X_{1} \times X_{2}} u_{\alpha_{1}}\left(x_{1}\right) v_{\alpha_{2}}\left(x_{2}\right) \varphi\left(x_{1}, x_{2}\right) d v_{1}\left(x_{1}\right) d v_{2}\left(x_{2}\right)\right| \\
\leqslant & \sum_{\alpha_{1}, \alpha_{2}}\left\|s_{1}^{\alpha_{1}} s_{2}^{\alpha_{2}}\right\|\left|\int_{X_{1}} u_{\alpha_{1}}\left(x_{1}\right) \varphi_{1}\left(x_{1}\right) d v_{1}\left(x_{1}\right) \int_{X_{2}} v_{\alpha_{2}}\left(x_{2}\right) \varphi_{2}\left(x_{2}\right) d v_{2}\left(x_{2}\right)\right| \\
\leqslant & \sum_{\alpha_{1}, \alpha_{2}}\left\|s_{1}\right\|^{\left|\alpha_{1}\right|} C_{1} r_{1}^{|\alpha|}\left\|\varphi_{1}\right\|_{C^{m_{1}}\left(X_{1}\right)}\left\|s_{2}\right\|^{\left|\alpha_{2}\right|} C_{2} r_{2}^{|\alpha|}\left\|\varphi_{2}\right\|_{C^{m_{2}}\left(X_{2}\right)} \\
\leqslant & \sum_{\alpha_{1}, \alpha_{2}} C_{1}\left(\left\|s_{1}\right\| r_{1}\right)^{\left|\alpha_{1}\right|} C_{2}\left(\left\|s_{2}\right\| r_{2}\right)^{\left|\alpha_{2}\right|}\|\varphi\|_{C^{m}\left(X_{1} \times X_{2}\right)}
\end{aligned}
$$

for any $m \geqslant \sup \left(m_{1}, m_{2}\right)$ where the r.h.s is absolutely convergent for $s_{1}, s_{2}$ small enough. Then we conclude by using the fact that the completed tensor product $C_{K_{1}}^{\infty}\left(X_{1}\right) \widehat{\otimes} C_{K_{2}}^{\infty}\left(X_{2}\right)$ coincides with $C_{K_{1} \times K_{2}}^{\infty}\left(X_{1} \times X_{2}\right)$ where the topology for which we do the completion does not matter since $C_{K_{i}}^{\infty}\left(X_{i}\right)$ are Fréchet nuclear spaces. Therefore the algebraic tensor product $C_{K_{1}}^{\infty}\left(X_{1}\right) \otimes C_{K_{2}}^{\infty}\left(X_{2}\right)$ is dense in $C_{K_{1} \times K_{2}}^{\infty}\left(X_{1} \times X_{2}\right)$ and the inequality

$$
\left|\sum_{\alpha_{1}, \alpha_{2}} s_{1}^{\alpha_{1}} s_{2}^{\alpha_{2}} \int_{X_{1} \times X_{2}} u_{\alpha_{1}}\left(x_{1}\right) v_{\alpha_{2}}\left(x_{2}\right) \varphi\left(x_{1}, x_{2}\right) d v_{1}\left(x_{1}\right) d v_{2}\left(x_{2}\right)\right| \leqslant \sum_{\alpha_{1}, \alpha_{2}} C_{1}\left(\left\|s_{1}\right\| r_{1}\right)^{\left|\alpha_{1}\right|} C_{2}\left(\left\|s_{2}\right\| r_{2}\right)^{\left|\alpha_{2}\right|}\|\varphi\|_{C^{m}\left(X_{1} \times X_{2}\right)}
$$

holds true for all $\varphi \in C_{K_{1} \times K_{2}}^{\infty}\left(X_{1} \times X_{2}\right)$.

For every $p \in \mathbb{C}^{p}, s_{0} \in \mathbb{R}^{p} \subset \mathbb{C}^{p}$, let $\pi_{p}: \mathcal{D}^{\prime}\left(M, \mathcal{M}_{s_{0}}\left(\mathbb{C}^{p}\right)\right) \mapsto \mathcal{D}^{\prime}\left(M, \mathcal{O}_{s_{0}}\left(\mathbb{C}^{p}\right)\right)$ be the projection from Proposition 6.2 . Then :

Lemma 7.2 Under the assumptions of the previous Lemma, the following equation holds true:

$$
\pi_{p_{1}+p_{2}}\left(t_{1} \otimes t_{2}\right)=\pi_{p_{1}}\left(t_{1}\right) \otimes \pi_{p_{2}}\left(t_{2}\right) .
$$

Proof - The proof of equation 35 goes as follows, we decompose $t_{1}$ and $t_{2}$ as $t_{1}=\pi_{p_{1}}\left(t_{1}\right)+\left(1-\pi_{1}\right)\left(t_{1}\right)$ and $t_{2}=\pi_{p_{2}}\left(t_{2}\right)+\left(1-\pi_{2}\right)\left(t_{2}\right)$ where $\left(\pi_{p_{1}}\left(t_{1}\right), \pi_{p_{2}}\left(t_{2}\right)\right) \in \mathcal{D}^{\prime}\left(X_{1}, \mathcal{O}_{\mu_{1}}\left(\mathbb{C}^{p_{1}}\right)\right) \times \mathcal{D}^{\prime}\left(X_{2}, \mathcal{O}_{\mu_{2}}\left(\mathbb{C}^{p_{2}}\right)\right)$ and $\left(\left(1-\pi_{1}\right)\left(t_{1}\right),\left(1-\pi_{2}\right)\left(t_{2}\right)\right) \in \mathcal{D}^{\prime}\left(X_{1}, \mathcal{P}_{\mu_{1}}\left(\mathbb{C}^{p_{1}}\right)\right) \times \mathcal{D}^{\prime}\left(X_{2}, \mathcal{P}_{\mu_{2}}\left(\mathbb{C}^{p_{2}}\right)\right)$. Then note that

$$
\begin{aligned}
t_{1} \otimes t_{2} & =\underbrace{\pi_{p_{1}}\left(t_{1}\right) \otimes \pi_{p_{2}}\left(t_{2}\right)}_{\in \mathcal{D}^{\prime}\left(X_{1} \times X_{2}, \mathcal{O}_{\left(\mu_{1}, \mu_{2}\right)}\left(\mathbb{C}^{p_{1}+p_{2}}\right)\right)} \\
& +\underbrace{\left(1-\pi_{1}\right)\left(t_{1}\right) \otimes \pi_{2}\left(t_{2}\right)+\pi_{1}\left(t_{1}\right) \otimes\left(1-\pi_{2}\right)\left(t_{2}\right)+\left(1-\pi_{1}\right)\left(t_{1}\right) \otimes\left(1-\pi_{2}\right)\left(t_{2}\right)}_{\in \mathcal{D}^{\prime}\left(X_{1} \times X_{2}, \mathcal{P}_{\left(\mu_{1}, \mu_{2}\right)}\left(\mathbb{C}^{p_{1}+p_{2}}\right)\right)}
\end{aligned}
$$

where the term $\left(1-\pi_{1}\right)\left(t_{1}\right) \otimes \pi_{2}\left(t_{2}\right)+\pi_{1}\left(t_{1}\right) \otimes\left(1-\pi_{2}\right)\left(t_{2}\right)+\left(1-\pi_{1}\right)\left(t_{1}\right) \otimes\left(1-\pi_{2}\right)\left(t_{2}\right)$ is a finite sum of polar germs by equation (32). It follows that $\pi_{p_{1}+p_{2}}\left(t_{1} \otimes t_{2}\right)=\pi_{p_{1}}\left(t_{1}\right) \otimes \pi_{p_{2}}\left(t_{2}\right)$ by the uniqueness of the decomposition which follows from Theorem 6.1

By a similar proof as in the above Lemma, we also have :

Lemma 7.3 Let $X$ be a smooth manifold, $U \subset X$ an open subset and $m \in \mathbb{N}$. Set $\left(p_{1}, p_{2}\right) \in \mathbb{N}^{2}$ to be an arbitrary pair of integers and $\left(\mu_{1}, \mu_{2}\right) \in \mathbb{R}^{p_{1}} \times \mathbb{R}^{p_{2}} \subset \mathbb{C}^{p_{1}+p_{2}}$. Let $t\left(s_{1}\right) \in \mathcal{D}^{\prime, m}\left(U, \mathcal{M}_{\mu_{1}}\left(\mathbb{C}^{p_{1}}\right)\right)$ and $h\left(s_{2}\right) \in C^{m}\left(U, \mathcal{O}_{\mu_{2}}\left(\mathbb{C}^{p_{2}}\right)\right)$. Then the product $t\left(s_{1}\right) h\left(s_{2}\right)$ is an element of $\mathcal{D}^{\prime, m}\left(U, \mathcal{M}_{\left(\mu_{1}, \mu_{2}\right)}\left(\mathbb{C}^{p_{1}+p_{2}}\right)\right)$ which satisfies the equation:

$$
\pi_{p_{1}+p_{2}}\left(t\left(s_{1}\right) h\left(s_{2}\right)\right)=\pi_{p_{1}}\left(t\left(s_{1}\right)\right) h\left(s_{2}\right) \text {. }
$$


Proof - Without loss of generality, we can work locally since all local results can be glued together by partition of unity thanks to Lemma 4.2. For every test function $\varphi \in C_{c}^{\infty}(U),\left\langle t\left(s_{1}\right) h\left(s_{2}\right), \varphi\right\rangle=$ $\langle t\left(s_{1}\right), \underbrace{h\left(s_{2}\right) \varphi}_{\in C^{m}(U)}\rangle$ hence the product $t\left(s_{1}\right) h\left(s_{2}\right)$ is well defined in $\mathcal{D}^{\prime, m}(U)$ as soon as both $t\left(s_{1}\right), h\left(s_{2}\right)$

exist. We now explain the meromorphicity of $\left(s_{1}, s_{2}\right) \mapsto\left\langle t\left(s_{1}\right) h\left(s_{2}\right), \varphi\right\rangle$ at $\left(\mu_{1}, \mu_{2}\right) \in \mathbb{C}^{p_{1}+p_{2}}$. Since $t(s) \in \mathcal{D}^{\prime, m}\left(U, \mathcal{M}_{\mu_{1}}\left(\mathbb{C}^{p_{1}}\right)\right)$, there exists $u(s) \in \mathcal{D}^{\prime, m}\left(U, \mathcal{O}_{\mu_{1}}\left(\mathbb{C}^{p_{1}}\right)\right)$ and linear functions $\left(L_{1}, \ldots, L_{k}\right)$ such that $\left(L_{1}(s) \cdots L_{k}(s)\right) t(s)=u(s)$. Therefore the product $t\left(s_{1}\right) h\left(s_{2}\right)$ also reads $\frac{1}{L_{1}\left(s_{1}\right) \cdots L_{k}\left(s_{1}\right)} u\left(s_{1}\right) h\left(s_{2}\right)$. Then using power expansions in $s_{1}-\mu_{1}$ for $u\left(s_{1}\right)$ as in Theorem 3.2 and expanding $h\left(s_{2}\right)$ in powers of $s_{2}-\mu_{2}$ where coefficients are in $C^{m}(U)$, we easily show that $u\left(s_{1}\right) h\left(s_{2}\right) \in \mathcal{D}^{\prime, m}\left(U, \mathcal{O}_{\left(\mu_{1}, \mu_{2}\right)}\left(\mathbb{C}^{p_{1}+p_{2}}\right)\right)$ for $\left(s_{1}, s_{2}\right) \in \mathbb{C}^{p_{1}+p_{2}}$ close enough to $\left(\mu_{1}, \mu_{2}\right) \in \mathbb{C}^{p_{1}+p_{2}}$ which proves $t\left(s_{1}\right) h\left(s_{2}\right) \in \mathcal{D}^{\prime, m}\left(U, \mathcal{M}_{\left(\mu_{1}, \mu_{2}\right)}\left(\mathbb{C}^{p_{1}+p_{2}}\right)\right)$.

The equation $\pi_{p_{1}+p_{2}}(t h)=h \pi_{p_{1}}(t)$ immediately follows from the fact that $\pi_{p_{2}}\left(h\left(s_{2}\right)\right)=h\left(s_{2}\right)$ since $h$ is holomorphic and $h\left(s_{2}\right)\left(1-\pi_{p_{1}}\right)\left(t\left(s_{1}\right)\right)$ is valued in polar germs.

\subsection{Proof of Lemma 4.1 .}

Proof - Since our Riemannian manifold $(M, g)$ is connected, $\operatorname{ker}(P)$ contains only constant functions. Indeed $P u=0$ implies that $u \in C^{\infty}$ by elliptic regularity and $0=\left\langle u,-\Delta_{g} u\right\rangle+\langle u, V u\rangle \Longrightarrow\langle\nabla u, \nabla u\rangle=$ $0 \Longrightarrow \nabla u=0$ thus $u$ is constant on connected components. Let us determine explicitly the spectral projector $\Pi$, it should satisfy for all $u$ :

$$
0=\langle 1, u-\Pi(u)\rangle=\int_{M}(u-\Pi(u))=\int_{M} u d x-\Pi(u) \operatorname{Vol}(M) \Longrightarrow \Pi(u)=\frac{\int_{M} u d x}{\operatorname{Vol}(M)} .
$$

The Schwartz kernel of the spectral projector $\Pi$ is therefore the constant function $\Pi(x, y)=\operatorname{Vol}(M)^{-1}$.

The first two claims about the Schwartz kernel $\mathfrak{G}^{s}(x, y)$ follow from [69, Theorem 4 p. 302] in the celebrated work of Seeley, by applying his Theorem to $A=P-\Pi$ which is a well defined elliptic pseudodifferential operator of order 2 .

For the third claim, we start from the formula $\mathfrak{G}^{s}=\int_{0}^{\infty}\left(e^{-t P}-\Pi\right)(x, y) t^{s-1} d t$ and our proof exactly follows the proof of [7, Proposition 1] where we replace the heat semigroup $e^{t \Delta_{g}}$ in their proof by the semigroup $\left(e^{-t P}-\Pi\right)$ whose Schwartz kernel is $K_{t}-\Pi$ and is denoted by $p_{t}$. Start from the formula $p_{t}(x, y)=\left\langle\delta_{x},\left(e^{-t P}-\Pi\right) \delta_{y}\right\rangle_{L^{2}(M)}=\left\langle\left(e^{-\frac{t}{2} P}-\Pi\right) \delta_{x},\left(e^{-\frac{t}{2} P}-\Pi\right) \delta_{y}\right\rangle_{L^{2}(M)}$. For any integers $(k, l, m),\left|\partial_{t}^{m} P_{x}^{k} P_{y}^{l} p_{t}(x, y)\right|=\left|P_{x}^{k+m} P_{y}^{l} p_{t}(x, y)\right|$ since $\partial_{t}^{m}\left(e^{-t P}-\Pi\right)=P^{m}\left(e^{-t P}-\Pi\right)$. Hence,

$$
\left|\partial_{t}^{m} P_{x}^{k} P_{y}^{l} p_{t}(x, y)\right| \leqslant\left\|\left(e^{-(t-\varepsilon) P}-\Pi\right)\right\|_{B\left(L^{2}(M)\right)}\left\|P_{x}^{k+m}\left(e^{-\frac{\varepsilon}{2} P}-\Pi\right) \delta_{x}\right\|_{L^{2}(M)}\left\|P_{y}^{l}\left(e^{-\frac{\varepsilon}{2} P}-\Pi\right) \delta_{y}\right\|_{L^{2}(M)} .
$$

Therefore taking the supremum over $(x, y) \in M \times M$ yields :

$$
\left\|\partial_{t}^{m} P_{x}^{k} P_{y}^{l} p_{t}\right\|_{C^{0}(M \times M)} \leqslant\left\|\left(e^{-(t-\varepsilon) P}-\Pi\right)\right\|_{B\left(L^{2}(M)\right)}\left\|P_{x}^{k+m}\left(e^{-\frac{\varepsilon}{2} P}-\Pi\right) \delta_{x}\right\|_{L^{2}(M)}\left\|P_{y}^{l}\left(e^{-\frac{\varepsilon}{2} P}-\Pi\right) \delta_{y}\right\|_{L^{2}(M)}
$$

where both $\left\|P_{x}^{k+m}\left(e^{-\frac{\varepsilon}{2} P}-\Pi\right) \delta_{x}\right\|_{L^{2}(M)}$ and $\left\|P_{y}^{l}\left(e^{-\frac{\varepsilon}{2} P}-\Pi\right) \delta_{y}\right\|_{L^{2}(M)}$ are finite since both $\left(e^{-\frac{\varepsilon}{2} P}-\right.$ $\Pi) \delta_{x}$ and $\left(e^{-\frac{\varepsilon}{2} P}-\Pi\right) \delta_{y}$ are smooth functions because the semigroup $\left(e^{t P}-\Pi\right)_{t \in \mathbb{R}_{\geqslant 0}}$ is smoothing. Furthermore, the term $\left\|\left(e^{-(t-\varepsilon) P}-\Pi\right)\right\|_{B\left(L^{2}(M)\right)}$ has exponential decay when $t \rightarrow+\infty$ since $\left(e^{-(t-\varepsilon) P}-\Pi\right)$ is a smoothing operator which has a gap in the spectrum, indeed by spectral theory $e^{-t P} u=\sum_{\lambda \in \sigma(P)} e^{-t \lambda} \Pi_{\lambda}(u)$ where $\Pi_{\lambda}$ is the spectral projector on the eigenspace of eigenvalue $\lambda$ and the r.h.s. converges absolutely in all Sobolev spaces $H^{s}(M), s \geq 0$ when $t>0$. More generally, we obtain decay estimates of the form

$$
\begin{aligned}
\left\|\partial_{t}^{m} p_{t}\right\|_{C^{k}(M \times M)} & \leqslant \sum_{l_{1}, l_{2} \leqslant \frac{k}{2}+1}\left\|\partial_{t}^{m} P_{x}^{k_{1}} P_{y}^{k_{2}} p_{t}\right\|_{C^{0}(M \times M)} \\
& \leqslant C_{k, m}\left\|\left(e^{-(t-\varepsilon) P}-\Pi\right)\right\|_{B\left(L^{2}(M)\right)} \leqslant C_{k, m} e^{-(t-\varepsilon) \lambda_{1}}
\end{aligned}
$$


where $\lambda_{1}>0$ is the smallest non zero eigenvalue of $P$ which exists since $\sigma(P)$ is a discrete subset of $[0,+\infty)$. It follows that the integral $\int_{1}^{\infty} t^{s-1} p_{t} d t$ converges absolutely for all $s \in \mathbb{C}$ and is valued in all Banach spaces $C^{k}(M \times M), k \in \mathbb{N}$ since :

$$
\left\|\int_{1}^{\infty} t^{s-1} p_{t} d t\right\|_{C^{k}(M \times M)} \leqslant \int_{1}^{\infty} t^{R e(s)-1}\left\|p_{t}\right\|_{C^{k}(M \times M)} d t \leqslant C_{k} \int_{1}^{\infty} t^{R e(s)-1} e^{-(t-\varepsilon) \lambda_{1}} d t .
$$

The integral $\int_{1}^{\infty} t^{s-1} p_{t} d t$ depends holomorphically in $s$ since

$$
\left\|\int_{1}^{\infty}\left(\frac{d}{d s}\right)^{l} t^{s-1} p_{t} d t\right\|_{C^{k}(M \times M)} \leqslant C_{k} \int_{1}^{\infty} t^{R e(s)-1} \log (t)^{l} e^{-(t-\varepsilon) \lambda_{1}} d t
$$

where the r.h.s. is absolutely convergent and we can conclude by dominated convergence arguments.

\subsection{Proof of Lemma 5.5 .}

Proof - First notice that when $\operatorname{Re}\left(s_{i}\right)>-1, i=1, \cdots, E$, this integral is absolutely convergent and holomorphic in $s$.

Now If $E=1$, then by integration by parts, for $\operatorname{Re}(s)>-1$,

$$
\int_{[0,1]} t^{s} \psi(t) d t=\sum_{i=0}^{k-1}(-1)^{i} \frac{1}{l_{i}(s)} \psi^{(i)}(1)+(-1)^{k} \frac{1}{l_{k-1}(s)} \int_{[0,1]} t^{s+k} \psi^{(k)}(t) d t,
$$

where $l_{i}(s)=(s+1) \cdots(s+i+1)$, the l.h.s is a meromorphic function when $\operatorname{Re}(s)>-k-1$ with possible poles at $s=-1, \cdots,-k$, so it extends to a meromorphic function on $\operatorname{Re}(s)>-k-1$.

In general, for $\operatorname{Re}\left(s_{i}\right)>-1, i=1, \cdots, E$, and $k_{1}, \cdots, k_{E} \in \mathbb{Z}_{>0}$,

$$
\begin{aligned}
I_{s}(\psi)= & \int_{[0,1]^{E}} t_{1}^{s_{1}} \ldots t_{E}^{s_{E}} \psi\left(t_{1}, \ldots, t_{E}\right) d^{E} t \\
= & \sum_{\left\{j_{1}, \cdots, j_{m}\right\} \subset\{1, \cdots, E\}} \sum_{\substack{j \neq j_{1}, \cdots, j_{m} \\
i_{j}=0, \cdots, k_{j}-1}} \frac{(-1)^{i_{j}}}{l_{i_{j}}\left(s_{j}\right)} \prod^{m} \frac{(-1)^{k_{j_{i}}}}{l_{k_{j_{i}}-1}\left(s_{j_{i}}\right)} \\
& \left.\int_{[0,1]^{m}} \prod_{j=j_{1}, \cdots j_{m}} t^{s_{j}+k_{j}}\left(\prod_{j \neq j_{1}, \cdots j_{m}} \partial_{t_{j}}^{i_{j}}\right) \partial_{t_{j_{1}}}^{k_{j_{1}}} \cdots \partial_{t_{j_{m}}}^{k_{j_{m}}} \psi\right|_{t_{j}=1, j \neq j_{1}, \cdots j_{m}} d t_{j_{1}} \cdots d t_{j_{s}}
\end{aligned}
$$

the r.h.s is a meromorphic function when $\operatorname{Re}\left(s_{i}\right)>-k_{i}-1$. So $I_{s}(\psi)$ extends to a meromorphic germ at any point in $\mathbb{Z}^{E}$.

Now at a given point $\left(p_{e}\right)_{e} \in \mathbb{Z}^{E}, \frac{1}{s_{e}-a_{e}}$ is holomorphic except $a_{e}=p_{e}$, therefore

$$
\left(\prod_{i \in I}\left(s_{i}-p_{i}\right)\right) I_{s}(\psi)
$$

is a holomorphic germ at $\left(p_{e}\right)_{e}$. The distribution order of $I_{s}(\psi)$ at the point $\left(p_{e}\right)$ can be read from Equation 38 easily.

\subsection{Proof of Lemma 5.2 .}

Proof - In the chart $\left(U \times U,\left(x^{\mu}, y^{\nu}\right)\right)$, let us consider the Taylor expansion of $\phi(x, y), \phi(x, y)=$ $\sum_{k \geqslant 0} \phi_{[k]}(x, y)$, where $\phi_{[k]}(x, y)=\sum_{|\alpha|+|\beta|=k} \frac{x^{\alpha} y^{\beta}}{\alpha ! \beta !} \partial_{x}^{\alpha} \partial_{y}^{\beta} \phi(0,0)$.

Obviously $\phi_{[0]}(x, y)=0$. By symmetry and $\phi(x, x)=0$, we know that

$$
\phi_{[1]}(x, y)=0 .
$$


By symmetry and $\phi(x, x) \equiv 0$, we know that $\phi_{[2]}(x, y)=\sum_{\mu} a_{\mu}\left(x^{\mu}-y^{\mu}\right)^{2}$, now by the fact $\phi(0, y)=$ $\|y\|^{2}$, we find that :

$$
\phi_{[2]}(x, y)=\sum\left(x^{\mu}-y^{\mu}\right)^{2} .
$$

In fact, let us take $x=y$ in Equation 19 ,

$$
g^{-1}(x)\left(\frac{\partial \phi}{\partial x^{\mu}}(x, x) d x^{\mu}, \frac{\partial \phi}{\partial x^{\nu}}(x, x) d x^{\nu}\right)=0
$$

which means

$$
\frac{\partial \phi}{\partial x^{\mu}}(x, x) d x^{\mu} \equiv 0 \Rightarrow \frac{\partial \phi}{\partial x^{\mu}}(x, x) \equiv 0
$$

By symmetry

$$
\frac{\partial \phi}{\partial y^{\mu}}(x, x) \equiv 0
$$

Now let us make a change of variables on $V \times W \rightarrow U \times U$ given by

$$
(v, h) \mapsto(v, v+h),
$$

we can take $V, W$ small enough such that $V \times W$ is a coordinate chart around $\left(x_{0}, x_{0}\right)$.

Let $\tilde{\phi}(v, h)=\phi(v, v+h)$. Take a partial Taylor expansion in $h$ for $\tilde{\phi}$,

$$
\tilde{\phi}(v, h)=\tilde{\phi}(v, 0)+\frac{\partial \tilde{\phi}}{\partial h^{\mu}}(v, 0) h^{\mu}+\frac{1}{2} \frac{\partial^{2} \tilde{\phi}}{\partial h^{\mu} \partial h^{\nu}}(v, 0) h^{\mu} h^{\nu}+\varepsilon_{3}
$$

where $\varepsilon_{3}$ vanishes at order 3 in $h$.

We know

$$
\tilde{\phi}(v, 0)=\phi(v, v)=0
$$

by Equation 40,

$$
\frac{\partial \tilde{\phi}}{\partial h^{\mu}}(v, 0)=\frac{\partial \phi}{\partial y^{\mu}}(v, v)=0
$$

By chain rule,

$$
\frac{\partial^{2} \tilde{\phi}}{\partial h^{\mu} \partial h^{\nu}}(v, 0)=\frac{\partial^{2} \phi}{\partial y^{\mu} \partial y^{\nu}}(v, v)
$$

Equation 19$]$ shows :

$$
\frac{\partial \phi}{\partial x^{\mu}}(x, y) g^{\mu \nu}(x) \frac{\partial \phi}{\partial x^{\nu}}(x, y)=4 \phi(x, y) .
$$

Taking $\frac{\partial^{2}}{\partial x^{\mu_{1}} \partial x^{\nu_{1}}}$ on both sides and let $x=y=v$, we have

$$
\frac{\partial^{2} \phi}{\partial x^{\mu} \partial x^{\mu_{1}}}(v, v) g^{\mu \nu}(v) \frac{\partial^{2} \phi}{\partial x^{\nu} \partial x^{\nu_{1}}}(v, v)+\frac{\partial^{2} \phi}{\partial x^{\mu} \partial x^{\nu_{1}}}(v, v) g^{\mu \nu}(v) \frac{\partial^{2} \phi}{\partial x^{\nu} \partial x^{\mu_{1}}}(v, v)=4 \frac{\partial^{2} \phi}{\partial x^{\mu_{1}} \partial x^{\nu_{1}}}(v, v) .
$$

that is

$$
\frac{\partial^{2} \phi}{\partial x^{\mu} \partial x^{\mu_{1}}}(v, v) g^{\mu \nu}(v) \frac{\partial^{2} \phi}{\partial x^{\nu} \partial x^{\nu_{1}}}(v, v)=2 \frac{\partial^{2} \phi}{\partial x^{\mu_{1}} \partial x^{\nu_{1}}}(v, v) .
$$

Notice that $\frac{\partial^{2} \phi}{\partial x^{\mu} \partial x^{\nu}}(v, v)$ is invertible since $\frac{\partial^{2} \phi}{\partial x^{\mu} \partial x^{\nu}}(0,0)=\delta_{\mu \nu}$ by 39 and if $U$ is chosen small enough. Then we get that

$$
\frac{\partial^{2} \phi}{\partial x^{\mu} \partial x^{\nu}}(v, v)=2 g_{\mu \nu}(v)
$$


Since $\phi$ is symmetry, we know

$$
\frac{\partial^{2} \phi}{\partial y^{\mu} \partial y^{\nu}}(v, v)=\frac{\partial^{2} \phi}{\partial x^{\mu} \partial x^{\nu}}(v, v)=2 g_{\mu \nu}(v) .
$$

So

$$
\tilde{\phi}(v, h)=g_{\mu \nu}(v) h^{\mu} h^{\nu}+\varepsilon_{3}
$$

which concludes the proof.

\section{References}

[1] Thomas Ackermann. A note on the Wodzicki residue. Journal of Geometry and Physics, 20(4):404406, 1996.

[2] Benjamin I Albert. Heat kernel renormalization on manifolds with boundary. arXiv preprint arXiv:1609.02220, 2016.

[3] Benjamin I. Albert. Effective field theory on manifolds with boundary. Ph.D. thesis, University of Pennsylvania, 2017.

[4] Bernd Ammann and Christian Bär. The Einstein-Hilbert Action as a Spectral Action. Noncommutative Geometry and the Standard Model of Elementary Particle Physics, pages 75-108, 2002.

[5] M. F. Atiyah. Resolution of singularities and division of distributions. Communications on pure and applied mathematics, 1970, vol. 23, no 2, p. 145-150.

[6] M. F. Atiyah, R. Bott, and V. K. Patodi. On the Heat Equation and the Index Theorem. Inventiones Math., 19:279-330, 1973.

[7] Christian Bär and Sergiu Moroianu. Heat kernel asymptotics for roots of generalized Laplacians. International Journal of Mathematics, 14(04):397-412, 2003.

[8] C Bär and A Strohmaier. An index theorem for Lorentzian manifolds with compact spacelike Cauchy boundary (2015). arXiv preprint arXiv:1506.00959.

[9] C. Bergbauer and D. Kreimer. The Hopf Algebra of Rooted Trees in Epstein-Glaser Renormalization. Annales Henri Poincaré Vol. 6. No. 2. Birkhäuser Basel, 2005, p. 343-367.

[10] Christoph Bergbauer, Romeo Brunetti, and Dirk Kreimer. Renormalization and resolution of singularities. arXiv preprint arXiv:0908.0633, 2009.

[11] Marko Berghoff. Wonderful Renormalization. PhD thesis, Humboldt-Universität zu Berlin, Mathematisch-Naturwissenschaftliche Fakultät, 2015.

[12] Marko Berghoff. Wonderful Compactifications in Quantum Field Theory. Communications in Number Theory and Physics, 9(3):477-547, 2015.

[13] Nicole Berline and Michèle Vergne. Local asymptotic Euler-Maclaurin expansion for Riemann sums over a semi-rational polyhedron. In Configuration Spaces, pages 67-105. Springer, 2016.

[14] N. Berline, E. Getzler, and M. Vergne. Heat kernels and Dirac operators. Springer Verlag, Berlin, 2004.

[15] R. Brunetti and K. Fredenhagen. Microlocal Analysis and Interacting Quantum Field Theories: Renormalization on Physical Backgrounds. Commun. Math. Phys., 208:623-61, 2000. 
[16] Andrei A Bytsenko, G Cognola, V Moretti, S Zerbini, and E Elizalde. Analytic Aspects of Quantum Fields. World Scientific, 2003.

[17] A. Cardona, C. Ducourtioux, and S. Paycha. From tracial anomalies to anomalies in Quantum Field Theory. Communications in mathematical physics 242.1-2 (2003): 31-65.

[18] Ozgür Ceyhan and Matilde Marcolli. Feynman integrals and motives of configuration spaces. Communications in Mathematical Physics, pages 1-36, 2012.

[19] Pierre Clavier, Li Guo, Sylvie Paycha, and Bin Zhang. An algebraic formulation of the locality principle in renormalisation. arXiv preprint arXiv:1711.00884, 2017.

[20] A. Connes and D. Kreimer. Hopf Algebras, Renormalization and Noncommutative Geometry. Commun. Math. Phys., 199:203-42, 1998.

[21] A. Connes and D. Kreimer. Renormalization in Quantum Field Theory and the Riemann-Hilbert Problem I: The Hopf Algebra Structure of Graphs and the Main Theorem. Commun. Math. Phys., 210:249-73, 2000.

[22] A. Connes and D. Kreimer. Renormalization in Quantum Field Theory and the Riemann-Hilbert Problem II: the $\beta$-Function, Diffeomorphisms and the Renormalization Group. Commun. Math. Phys., 216:215-41, 2001.

[23] K. Costello. Renormalization and Effective Field Theory, volume 170 of Math. Surveys Mono. Amer. Math. Soc., Providence, 2011.

[24] Nguyen Viet Dang. Complex powers of analytic functions and meromorphic renormalization in QFT. arXiv preprint arXiv:1503.00995, 2015.

[25] Jan Dereziński and Daniel Siemssen. Feynman propagators on static spacetimes. arXiv preprint arXiv:1608.06441, 2016.

[26] Jan Dereziński and Daniel Siemssen. An evolution equation approach to the Klein-Gordon operator on curved spacetime. arXiv preprint arXiv:1709.03911, 2017.

[27] J. J. Duistermaat. Fourier Integral Operators. Birkhäuser, Boston, 1996.

[28] H. Epstein and V. Glaser. The role of locality in perturbation theory. Ann. Inst. Henri Poincaré, 19:211-95, 1973.

[29] Stephen A Fulling. Vacuum energy as spectral geometry. Symmetry, Integrability and Geometry. Methods and Applications, 3, 2007.

[30] Christian Gérard and Michał Wrochna. The massive Feynman propagator on asymptotically Minkowski spacetimes. arXiv preprint arXiv:1609.00192, 2016.

[31] Christian Gérard and Michal Wrochna. Feynman propagators and Hadamard states from scattering data for the Klein-Gordon equation on asymptotically Minkowski spacetimes. arXiv preprint arXiv:1603.07465, 2016.

[32] Christian Gérard and Michał Wrochna. Analytic Hadamard states, Calderón projectors and Wick rotation near analytic Cauchy surfaces. arXiv preprint arXiv:1706.08942, 2017.

[33] Christian Gérard, Omar Oulghazi, and Michał Wrochna. Hadamard states for the KleinGordon equation on Lorentzian manifolds of bounded geometry. Communications in Mathematical Physics, 352(2):519-583, 2017.

[34] P Gilkey. Invariance Theory, the Heat Equation and the Atiyah-Singer Index Theorem, (1995). Studies in Advanced Mathematics, CRC Press, Inc. 
[35] Alexandre Grothendieck. Sur certains espaces de fonctions holomorphes. I. J. reine angew. Math, 192(1):35-64, 1953.

[36] Razvan Gurau, Vincent Rivasseau, and Alessandro Sfondrini. Renormalization: an advanced overview. arXiv preprint arXiv:1401.5003, 2014.

[37] R Gunning and H Rossi. Analytic functions of several complex variables. Prentice-Hall, Englewood Cliffs, NJ, 1965.

[38] Li Guo, Sylvie Paycha, and Bin Zhang. A conical approach to Laurent expansions for multivariate meromorphic germs with linear poles. arXiv preprint arXiv:1501.00426, 2015.

[39] Li Guo, Sylvie Paycha, and Bin Zhang. Counting an infinite number of points: a testing ground for renormalization methods. In Geometric, Algebraic, and Topological Methods for Quantum Field Theory: Proceedings of the 2013 Villa de Leyva Summer School, Villa de Leyva, Colombia, 15-27 July 2013, pages 309-352. World Scientific, 2017.

[40] Martin Hairer. An analyst's take on the BPHZ theorem. arXiv preprint arXiv:1704.08634, 2017.

[41] Stephen W Hawking. Zeta Function Regularization of Path Integrals in Curved Spacetime. Communications in Mathematical Physics, 55(2):133-148, 1977.

[42] E. Herscovich. Renormalization in Quantum Field Theory (after R. Borcherds).

[43] S. Hollands and R. M. Wald. Local Wick Polynomials and Time Ordered Products of Quantum Fields in Curved Spacetime. Commun. Math. Phys., 223:289-326, 2001.

[44] S. Hollands and R. M. Wald. Existence of Local Covariant Time Ordered Products of Quantum Fields in Curved Spacetime. Commun. Math. Phys., 231:309-45, 2002.

[45] W Kalau and M Walze. Gravity, non-commutative geometry and the Wodzicki residue. Journal of Geometry and Physics, 16(4):327-344, 1995.

[46] Daniel Kastler. The Dirac Operator and Gravitation. Communications in Mathematical Physics, 166(3):633-643, 1995.

[47] Maxim Kontsevich and Simeon Vishik. Determinants of elliptic pseudo-differential operators. arXiv preprint hep-th/9404046, 1994.

[48] Thomas Krajewski, Vincent Rivasseau, Adrian Tanasă, and Zhituo Wang. Topological Graph Polynomials and Quantum Field Theory part I: Heat Kernel Theories. Journal of Noncommutative Geometry, 4(1):29-82, 2010.

[49] Joseph B Kruskal. On the shortest spanning subtree of a graph and the traveling salesman problem. Proceedings of the American Mathematical society, 7(1):48-50, 1956.

[50] Solomon Lefschetz. Applications of algebraic topology. Springer, 1975.

[51] Matthias Lesch. On the Noncommutative Residue for Pseudodifferential Operators with logPolyhomogeneous Symbols. Annals of global analysis and geometry, 17(2):151-187, 1999.

[52] R. B. Melrose, The Atiyah-Patodi-Singer index theorem, AK Peters, Wellesley, 1993.

[53] R. B. Melrose and V. Nistor. Homology of pseudodifferential operators I. Manifolds with boundary. arXiv preprint funct-an/9606005 (1996).

[54] Pavel Mnev. Lecture notes on torsions. arXiv preprint arXiv:1406.3705, 2014. 
[55] Liviu I Nicolaescu. Random Morse functions and spectral geometry. arXiv preprint arXiv:1209.0639, 2012.

[56] Nikolay M Nikolov, Raymond Stora, and Ivan Todorov. Renormalization of massless Feynman amplitudes in configuration space. Reviews in Mathematical Physics, 26(04):1430002, 2014.

[57] Sylvie Paycha. Regularised Integrals, Sums and Traces. An analytic point of view, AMS University Lecture Notes, 59, 2012.

[58] Sylvie Paycha and Simon Scott. Chern-Weil forms associated with superconnections. Analysis, Geometry and Topology of elliptic operators, B. Booss-Bavnbeck, S. Klimek, M. Lesch, W. Zhang World Scientific, 2006.

[59] Sylvie Paycha and Simon Scott. A Laurent Expansion for Regularized Integrals of Holomorphic Symbols. GAFA Geometric And Functional Analysis, 17(2):491-536, 2007.

[60] G. Popineau and R. Stora. (2016). A pedagogical remark on the main theorem of perturbative renormalization theory. Nuclear Physics B, 912, 70-78.

[61] Steffen Pottel. A BPHZ theorem in configuration space. arXiv preprint arXiv:1706.06762, 2017.

[62] Steffen Pottel. Configuration space BPHZ renormalization on analytic spacetimes. arXiv preprint arXiv:1708.04112, 2017.

[63] Daniel B Ray and Isadore M Singer. R-torsion and the Laplacian on Riemannian manifolds. Advances in Mathematics, 7(2):145-210, 1971.

[64] Marcel Riesz. L'intégrale de Riemann-Liouville et le problème de Cauchy. Acta mathematica, 81(1):1-222, 1949.

[65] V. Rivasseau. From Perturbative to Constructive Renormalization. Princeton University Press, Princeton, 1991.

[66] John Roe. Elliptic operators, topology, and asymptotic methods. CRC Press, 1999.

[67] Albert S Schwarz. The partition function of degenerate quadratic functional and Ray-Singer invariants. Letters in Mathematical Physics, 2(3):247-252, 1978.

[68] Simon Scott. Traces and Determinants of Pseudodifferential Operators. OUP Oxford, 2010.

[69] Robert T Seeley. Complex powers of an elliptic operator. Electron. Res. Announc. Am. Math. Soc., pages 288-307, 1967.

[70] Eugene R Speer. Analytic Renormalization. Journal of Mathematical Physics, 9(9):1404-1410, 1968.

[71] Eugene R Speer. Dimensional and Analytic Renormalization. In Renormalization theory, pages 25-93. Springer, 1976.

[72] Eugene R Speer. Seminars on Renormalisation Theory, volume 1: Lectures on Analytic Renormalisation-University of Maryland-technical report 73-067 (1972). Dimensional and Analytic Renormalization.

[73] Michael Taylor. Partial Differential Equations II: Qualitative Studies of Linear Equations, volume 116. Springer Science \& Business Media, 2013.

[74] András Vasy and Michał Wrochna. Quantum fields from global propagators on asymptotically Minkowski and extended de Sitter spacetimes. arXiv preprint arXiv:1512.08052, 2015. 
[75] Mariusz Wodzicki. Noncommutative residue Chapter I. Fundamentals. In K-theory, arithmetic and geometry, pages 320-399. Springer, 1987.

[76] Mariusz Wodzicki. Local invariants of spectral asymmetry. Inventiones mathematicae, 75(1):143$177,1984$. 